\title{
The Kerr/CFT Correspondence and its Extensions
}

\author{
Geoffrey Compère \\ Physique Théorique et Mathématique \\ Université Libre de Bruxelles, CP 231, \\ 1050 Bruxelles, Belgium \\ email: gcompere@ulb.ac.be \\ http://www.ulb.ac.be/sciences/ptm/pmif/gcompere/
}

Accepted on 13 June 2012

Published on 22 October 2012

\begin{abstract}
We present a first-principles derivation of the main results of the Kerr/CFT correspondence and its extensions using only tools from gravity and quantum field theory, filling a few gaps in the literature when necessary. Firstly, we review properties of extremal black holes that imply, according to semi-classical quantization rules, that their near-horizon quantum states form a centrally-extended representation of the one-dimensional conformal group. This motivates the conjecture that the extremal Kerr and Reissner-Nordström black holes are dual to the chiral limit of a two-dimensional CFT. We also motivate the existence of an $S L(2, \mathbb{Z})$ family of twodimensional CFTs, which describe in their chiral limit the extremal Kerr-Newman black hole. We present generalizations in anti-de Sitter spacetime and discuss other matter-coupling and higher-derivative corrections. Secondly, we show how a near-chiral limit of these CFTs reproduces the dynamics of near-superradiant probes around near-extremal black holes in the semiclassical limit. Thirdly, we review how the hidden conformal symmetries of asymptotically-flat black holes away from extremality, combined with their properties at extremality, allow for a microscopic accounting of the entropy of non-extremal asymptotically-flat rotating or charged black holes. We conclude with a list of open problems.
\end{abstract}

This review is licensed under a Creative Commons Attribution-Non-Commercial-NoDerivs 3.0 Germany License. http://creativecommons.org/licenses/by-nc-nd/3.0/de/ 


\section{Imprint / Terms of Use}

Living Reviews in Relativity is a peer reviewed open access journal published by the Max Planck Institute for Gravitational Physics, Am Mühlenberg 1, 14476 Potsdam, Germany. ISSN 1433-8351.

This review is licensed under a Creative Commons Attribution-Non-Commercial-NoDerivs 3.0 Germany License: http://creativecommons.org/licenses/by-nc-nd/3.0/de/. Figures that have been previously published elsewhere may not be reproduced without consent of the original copyright holders.

Because a Living Reviews article can evolve over time, we recommend to cite the article as follows:

\section{Geoffrey Compère,}

"The Kerr/CFT Correspondence and its Extensions", Living Rev. Relativity, 15, (2012), 11. [Online Article]: cited [<date $>$, http://www.livingreviews.org/lrr-2012-11

The date given as $<$ date $>$ then uniquely identifies the version of the article you are referring to.

\section{Article Revisions}

Living Reviews supports two ways of keeping its articles up-to-date:

Fast-track revision A fast-track revision provides the author with the opportunity to add short notices of current research results, trends and developments, or important publications to the article. A fast-track revision is refereed by the responsible subject editor. If an article has undergone a fast-track revision, a summary of changes will be listed here.

Major update A major update will include substantial changes and additions and is subject to full external refereeing. It is published with a new publication number.

For detailed documentation of an article's evolution, please refer to the history document of the article's online version at http://www.livingreviews.org/lrr-2012-11. 


\section{Contents}

1 Introduction $\quad \mathbf{5}$

1.1 Classes of effective field theories . . . . . . . . . . . . . . . . . . . 7

1.2 Gauge fields as Kaluza-Klein vectors . . . . . . . . . . . . . . . . . 8

2 Extremal Black Holes as Isolated Systems 10

2.1 Properties of extremal black holes . . . . . . . . . . . . . . . . . . 10

2.2 Near-horizon geometries of static extremal black holes . . . . . . . . . . . . . . 13

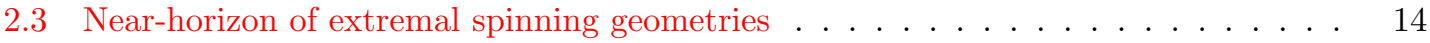

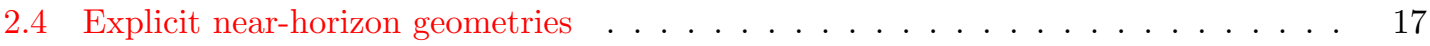

2.4.1 Near-horizon geometry of extremal Kerr . . . . . . . . . . . . . . . 17

2.4.2 Near-horizon geometry of extremal Reissner-Nordström . . . . . . . . . . 18

2.4.3 Near-horizon geometry of extremal Kerr-Newman . . . . . . . . . . . . 18

2.4.4 Near-horizon geometry of extremal Kerr-Newman-AdS . . . . . . . . . . 18

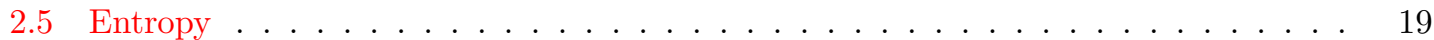

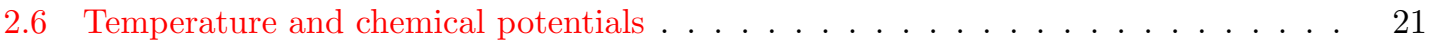

2.7 Near-extremal near-horizon geometries . . . . . . . . . . . . . . . . . . . . 24

2.8 Uniqueness of stationary near-horizon geometries . . . . . . . . . . . . . . . . . . . . . 26

2.9 Absence of bulk dynamics in near-horizon geometries . . . . . . . . . . . . . 26

3 Two-Dimensional Conformal Field Theories $\quad 28$

3.1 Cardy's formula . . . . . . . . . . . . . . . . . . . . . . . . . . . . . . . . . . . . . . . . . . . . . .

3.2 DLCQ and chiral limit of CFTs . . . . . . . . . . . . . . . . . . . . . . . . . . . . . . . . . . . . . . .

3.3 Long strings and symmetric orbifolds . . . . . . . . . . . . . . . . . 30

4 Microscopic Entropy of Extremal Black Holes $\quad 32$

4.1 Boundary conditions and asymptotic symmetry algebra . . . . . . . . . . . . . . . . 32

4.2 Absence of $S L(2, \mathbb{R})$ asymptotic symmetries . . . . . . . . . . . . . . . . . . . . . . . . . . . . . . . . . . . . .

4.3 Virasoro algebra and central charge . . . . . . . . . . . . . . . . . . . . . . . . . . . . . . . . . . . . .

4.4 Microscopic counting of the entropy . . . . . . . . . . . . . . . . . . 40

5 Scattering from Near-Extremal Black Holes $\quad \mathbf{4 3}$

5.1 Near-extremal Kerr-Newman black holes . . . . . . . . . . . . . . . . . . . 43

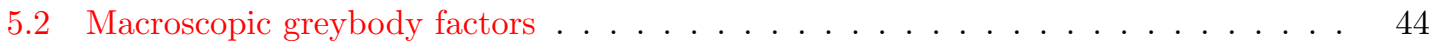

5.3 Macroscopic greybody factors close to extremality . . . . . . . . . . . . . . . . . 4 46

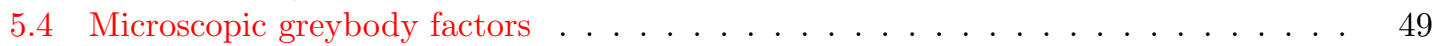

5.5 Microscopic accounting of superradiance . . . . . . . . . . . . . . . . 52

6 Hidden Symmetries of Non-Extremal Black Holes $\quad \mathbf{5 4}$

6.1 Scalar wave equation in Kerr-Newman . . . . . . . . . . . . . . . . . . . . . . 55

6.2 Scalar wave equation in Kerr-Newman-AdS . . . . . . . . . . . . . . . . . . 56

6.3 Near-region scalar-wave equation . . . . . . . . . . . . . . . . . . . . . . . . . . . . . . . . . 57

6.4 Local $S L(2, \mathbb{R}) \times S L(2, \mathbb{R})$ symmetries . . . . . . . . . . . . . . . . . . . . . . . . . . . . . . . . 67

6.5 Symmetry breaking to $U(1)_{L} \times U(1)_{R} \ldots \ldots \ldots \ldots$

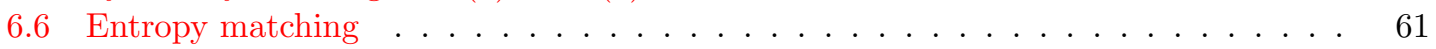

7 Summary and Open Problems $\quad 62$

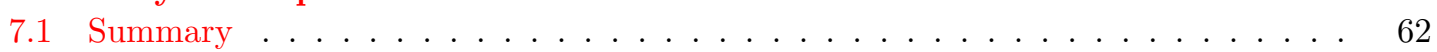

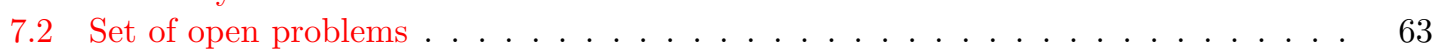


8 Acknowledgments 


\section{Introduction}

It is known since the work of Bardeen, Bekenstein, Carter and Hawking [42, 32, 162] that black holes are thermodynamical systems equipped with a temperature and an entropy. In analogy to Bolzmann's statistical theory of gases, one expects that the entropy of black holes counts microscopic degrees of freedom. Understanding what these degrees of freedom actually are is one of the main challenges that a theory of quantum gravity should address.

Since the advent of string theory, many black holes enjoying supersymmetry have been understood microscopically. In many cases, supersymmetry and its non-renormalization theorems allow one to map the black-hole states to dual states in a weakly-coupled description, which also provides a method to microscopically reproduce Hawking radiation; see [253, 60] and subsequent work. For all supersymmetric black holes that contain in their near-horizon limit a factor of three-dimensional anti-de Sitter spacetime $\mathrm{AdS}_{3}$ or a quotient thereof, a simpler microscopic model is available. Since quantum gravity in asymptotically $\mathrm{AdS}_{3}$ geometries is described by a two-dimensional conformal field theory $(2 d$ CFT) $[58,251]$, one can account for the entropy and the Hawking radiation of these supersymmetric or nearly supersymmetric black holes using only the universal properties of a dual CFT description defined in the near-horizon region [209, 104] (for reviews, see [155, 113]). Ultraviolet completions of these AdS/CFT correspondences can be constructed using string theory [205, 265].

These results can be contrasted with the challenge of describing astrophysical black holes that are non-supersymmetric and non-extremal, for which these methods cannot be directly applied. Astrophysical black holes are generically rotating and have approximately zero electromagnetic charge. Therefore, the main physical focus should be to understand the microstates of the Kerr black hole and to a smaller extent the microstates of the Schwarzschild, the Kerr-Newman and the Reissner-Nordström black hole.

Recently, considerable progress has been made in reproducing the entropy of the Kerr black hole as well as reproducing part of its gravitational dynamics using dual field theories that share many properties with two-dimensional CFTs [156, 53, 68] (see also [104]). ${ }^{1}$ The Kerr/CFT correspondence will be the main focus of this review. Its context is not limited to the sole Kerr black hole. Indeed, it turns out that the ideas underlying the correspondence apply as well to a large class of black holes in supergravity (in four and higher dimensions) independently of the asymptotic region (asymptotically-flat, anti-de Sitter...) far from the black hole. These extensions of the Kerr/CFT correspondence only essentially require the presence of a $U(1)$ axial symmetry associated with angular momentum. It is important to state that at present the Kerr/CFT correspondence and its extensions are most understood for extremal and near-extremal black holes. Only sparse but non-trivial clues point to a CFT description of black holes away from extremality [104, 68, 107].

Before jumping into the theory of black holes, it is important to note at the outset that rotating extremal black holes might be of astrophysical relevance. Assuming exactly zero electromagnetic charge, the bound on the Kerr angular momentum derived from the cosmic-censorship hypothesis is $J \leq G M^{2}$. No physical process exists that would turn a non-extremal black hole into an extremal one. Using details of the accretion disk around the Kerr black hole, Thorne derived the bound $J \leq 0.998 G M^{2}$ assuming that only reasonable matter can fall into the black hole [258]. Quite surprisingly, it has been claimed that several known astrophysical black holes, such as the black holes in the X-ray binary GRS 1905+105 [218] and Cygnus X-1 [152], are more than 95\% close to the extremality bound. Also, the spin-to-mass-square ratio of the supermassive black holes in the active galactic nuclei MCG-6-30-15 [57] and 1H 0707-495 [134] has been claimed to be around $98 \%$. However, these measurements are subject to controversy since independent data analyses based on different assumptions led to opposite results as reviewed in [138]: the spin-to-mass-

\footnotetext{
1 For independent arguments pointing to the presence of conformal symmetry around arbitrary black holes, see $[63,245,180]$. We will not discuss these approaches here.
} 
square ratio of the very same black hole in the X-ray binary GRS $1905+105$ has been evaluated as $J /\left(G M^{2}\right)=0.15$ [182], while the spin of the black hole in Cygnus X-1 has been evaluated as $J /\left(G M^{2}\right)=0.05$ [219]. If the measurements of high angular momenta are confirmed or if precise measurements of other nearby highly-spinning black holes can be performed, it would promote extremal black holes as "nearly physical" objects of nature.

In this review, we will present a derivation of the arguments underlying the Kerr/CFT correspondence and its extensions starting from first-principles. For that purpose, it will be sufficient to follow an effective field theory approach based solely on gravity and quantum field theory. In particular, we will not need any detail of the ultraviolet completions of quantum gravity except for one assumption (see Section 1.1 for a description of the precise classes of gravitational theories under study). We will assume that the $U(1)$ electromagnetic field can be promoted to be a Kaluza-Klein vector of a higher-dimensional spacetime (see Section 1.2 for some elementary justifications and elaborations on this assumption). If this assumption is correct, it turns out that the Kerr/CFT correspondence can be further generalized using the $U(1)$ electric charge as a key quantity instead of the $U(1)$ angular momentum [159]. We will use this assumption as a guiding principle to draw parallels between the physics of static charged black holes and rotating black holes. Our point of view is that a proper understanding of the concepts behind the Kerr/CFT correspondence is facilitated by studying in parallel static-charged black holes and rotating black holes.

Since extremal black holes are the key objects of study, we will spend a large amount of time describing their properties in Section 2. We will contrast the properties of static extremal black holes and of rotating extremal black holes. We will discuss how one can decouple the near-horizon region from the exterior region. We will then show that one can associate thermodynamical properties with any extremal black hole and we will argue that near-horizon geometries contain no local bulk dynamics. Since we aim at drawing parallels between black holes and two-dimensional CFTs, we will quickly review some of their most relevant properties for our concerns in Section 3.

After this introductory material, we will discuss the core of the Kerr/CFT correspondence starting from the microscopic counting of the entropy of extremal black holes in Section 4. There, we will show how the near-horizon region admits a set of symmetries at its boundary, which form a Virasoro algebra. Several choices of boundary conditions exist, where the algebra extends a different compact $U(1)$ symmetry of the black hole. Following semi-classical quantization rules, the operators, which define quantum gravity in the near-horizon region, form a representation of the Virasoro algebra. We will then argue that near-horizon quantum states can be identified with those of a chiral half of a two-dimensional CFT. This thesis will turn out to be consistent with the description of non-extremal black holes. The thermodynamical potential associated with the $U(1)$ symmetry will then be interpreted as the temperature of the density matrix dual to the black hole. The entropy of the black hole will finally be reproduced from the asymptotic growth of states in each chiral half of these CFTs via Cardy's formula.

In Section 5 we will move to the description of non-extremal black holes, and we will concentrate our analysis on asymptotically-flat black holes for simplicity. We will describe how part of the dynamics of probe fields in the near-extremal Kerr-Newman black hole can be reproduced by correlators in a family of dual CFTs with both a left and a right-moving sector. The left-moving sector of the CFTs will match with the corresponding chiral limit of the CFTs derived at extremality. In Section 6 we will review the hidden local conformal symmetry that is present in some probes around the generic Kerr-Newman black hole. We will also infer from the breaking of this conformal symmetry that the Kerr-Newman black hole entropy can be mapped to states of these CFTs at specific left and right-moving temperatures. Finally, we will summarize the key results of the Kerr/CFT correspondence in Section 7 and provide a list of open problems. This review complements the lectures on the Kerr black hole presented in [54] by providing an overview of the Kerr/CFT correspondence and its extensions for general rotating or charged black holes in gravity coupled to matter fields in a larger context. Since we follow an effective field-theory approach, we

Living Reviews in Relativity

http://www. livingreviews.org/lrr-2012-11 
will cover string-theory models of black holes only marginally. We refer the interested reader to the complementary string theory-oriented review of extremal black holes [244]. ${ }^{2}$

\subsection{Classes of effective field theories}

The Kerr/CFT correspondence is an effective description of rotating black holes with an "infrared" CFT. Embedding this correspondence in string theory has the potential to give important clues on the nature of the dual field theory. Efforts in that direction include $[225,22,157,98,23,116,31$, 243, 246, 115, 130]. However, the details of particular CFTs are irrelevant for the description of astrophysical black holes, as long as we don't have a reasonable control of all realistic embeddings of the standard model of particle physics and cosmology in string theory. Despite active research in this area, see, e.g., [153, 117, 217], a precise description of how our universe fits in to the landscape of string theory is currently out-of-reach.

In an effective field theory approach, one concentrates on long-range interactions, which are described by the physical Einstein-Maxwell theory. However, it is instructive in testing ideas about quantum gravity models of black holes to embed our familiar Einstein-Maxwell theory into the larger framework of supergravity and study the generic properties of rotating black holes as toy models for a physical string embedding of the Kerr-Newman black hole.

Another independent motivation comes from the AdS/CFT correspondence [205, 265]. Black holes in anti-de Sitter (AdS) spacetime in $d+1$ dimensions can be mapped to thermal states in a dual CFT or CFT in $d$ dimensions. Studying AdS black holes then amounts to describing the dynamics of the dual strongly-coupled CFT in the thermal regime. Since this is an important topic, we will discuss in this review the AdS generalizations of the Kerr/CFT correspondence as well. How the Kerr/CFT correspondence fits precisely in the AdS/CFT correspondence in an important open question that will be discussed briefly in Section 7.2.

In this review we will consider the following class of four-dimensional theories,

$$
\begin{aligned}
S=\frac{1}{16 \pi G} \int & d^{D} x \sqrt{-g}\left(R-\frac{1}{2} f_{A B}(\chi) \partial_{\mu} \chi^{A} \partial^{\mu} \chi^{B}-V(\chi)-k_{I J}(\chi) F_{\mu \nu}^{I} F^{J \mu \nu}\right. \\
& \left.+h_{I J}(\chi) \epsilon^{\mu \nu \rho \sigma} F_{\mu \nu}^{I} F_{\rho \sigma}^{J}\right),
\end{aligned}
$$

possibly supplemented with Planck-suppressed higher-derivative corrections. We focus on the case where $f_{A B}(\chi)$ and $k_{I J}(\chi)$ are positive definite and the scalar potential $V(\chi)$ is non-positive in (1). This ensures that matter obeys the usual energy conditions and it covers the case of zero and negative cosmological constant. Some theories of interest contained in the general class (1) are the Einstein-Maxwell gravity with negative or zero cosmological constant and the bosonic sector of $\mathcal{N}=8$ supergravity. Note that the phenomenology described by the action (1) is limited by the absence of charged scalars, massive vectors, non-abelian gauge fields and fermions.

The explicit form of the most general single-center spinning-black-hole solution of the theory (1) is not known;however, see [270, 221] for general ansätze. For Einstein and Einstein-Maxwell theory, the solutions are, of course, the Kerr and Kerr-Newman geometries that were derived about 45 years after the birth of general relativity. For many theories of theoretical interest, e.g., $\mathcal{N}=8$ supergravity, the explicit form of the spinning-black-hole solution is not known, even in a specific U-duality frame (see, e.g., [49] and references therein). However, as we will discuss in Section 2.3, the solution at extremality greatly simplifies in the near-horizon limit due to additional symmetries

\footnotetext{
${ }^{2}$ Nevertheless, let us mention that some classes of black holes admit a vanishing horizon area $A_{h}$ and zero temperature $T$ limit such that the ratio $A_{h} / T$ is finite. Such extremal vanishing horizon (EVH) black holes admit near-horizon limits, which contain (singular) identifications of $\mathrm{AdS}_{3}$ that can be used for string model building [157, 98, 116, 130, 115]. Most of the ideas developed for the Kerr/CFT correspondence and its extensions can be developed similarly for EVH black holes [243].
} 
and takes a universal form for any theory in the class (1). It is for this reason mainly that we find convenient to discuss theory (1) in one swoop.

\subsection{Gauge fields as Kaluza-Klein vectors}

Since the work of Kaluza and Klein, one can conceive that our $U(1)$ electromagnetic gauge field could originate from a Kaluza-Klein vector of a higher-dimensional spacetime of the form $\mathcal{M}_{4} \times X$, where $\mathcal{M}_{4}$ is our spacetime, $X$ is compact and contains at least a $U(1)$ cycle (the total manifold might not necessarily be a direct product). Experimental constraints on such scenarios can be set from bounds on the deviation of Newton's law at small scales [197, 2].

If our $U(1)$ electromagnetic gauge field can be understood as a Kaluza-Klein vector, it turns out that it is possible to account for the entropy of the Reissner-Nordström black hole in essentially the same way as for the Kerr black hole [159]. This mainly follows from the fact that the electric charge becomes an angular momentum $J_{2}=Q$ in the higher-dimensional spacetime, which is on the same footing as the four-dimensional angular momentum $J_{1}=J$ lifted in the higher-dimensional spacetime.

Assumption We will assume throughout this review that the $U(1)$ electromagnetic gauge field can be promoted as a Kaluza-Klein vector.

As far as the logic goes, this assumption will not be required for any reasoning in Section 2, even though it will help to understand striking similarities between the effects of rotation and electric charge. The assumption will be a crucial input in order to formulate the Reissner-Nordström/CFT correspondence and its generalizations in Section 4 and further on. This assumption is not required for the Kerr/CFT correspondence and its (extremal or non-extremal) extensions, which are exclusively based on the $U(1)$ axial symmetry of spinning black holes.

In order to make this idea more precise, it is important to study simple embeddings of the $U(1)$ gauge field in higher-dimensional spacetimes as toy models for a realistic embedding. In asymptotically-flat spacetimes, let us introduce a fifth compact dimension $\chi \sim \chi+2 \pi R_{\chi}$, where $2 \pi R_{\chi}$ is the length of the $U(1)$ Kaluza-Klein circle and let us define

$$
d s^{2}=d s_{(4)}^{2}+(d \chi+A)^{2} .
$$

The metric (2) does not obey five-dimensional Einstein's equations unless the metric is complemented by matter fields. One simple choice consists of adding a $U(1)$ gauge field $A_{(5)}$, whose field strength is defined as

$$
d A_{(5)}=\frac{\sqrt{3}}{2} \star_{(4)} F
$$

where $\star_{(4)}$ is the four-dimensional Hodge dual. The five-dimensional metric and gauge field are then solutions to the five-dimensional Einstein-Maxwell-Chern-Simons theory, as reviewed, e.g., in $[185]$.

These considerations can also be applied to black holes in anti-de Sitter spacetimes. However, the situation is more intricate because no consistent Kaluza-Klein reduction from five dimensions can give rise to the four-dimensional Einstein-Maxwell theory with cosmological constant [204]. As a consequence, the four-dimensional Kerr-Newman-AdS black hole cannot be lifted to a solution of any five-dimensional theory. Rather, embeddings in eleven-dimensional supergravity exist, which are obtained by adding a compact seven-sphere [69, 109].

Therefore, in order to review the arguments for the Reissner-Nordström/CFT correspondence and its generalizations, it is necessary to discuss five-dimensional gravity coupled to matter fields. We will limit our arguments to the action (1) possibly supplemented by the Chern-Simons terms

$$
S_{\mathrm{CS}}=\frac{1}{16 \pi G_{5}} \int d^{5} x C_{I J K} A^{I} \wedge F^{J} \wedge F^{K},
$$

Living Reviews in Relativity

http://www. livingreviews.org/lrr-2012-11 
where $C_{I J K}=C_{(I J K)}$ are constants. This theory will suffice to discuss in detail the embedding (2) - (3) since the five-dimensional Einstein-Maxwell-Chern-Simons theory falls into that class of theories. We will not discuss the supergravities required to embed AdS-Einstein-Maxwell theory.

Let us finally emphasize that even though the scale $R_{\chi}$ of the Kaluza-Klein direction is arbitrary as far as it allows one to perform the uplift (2), it is constrained by matter field couplings. For example, let us consider the toy model of a probe charged massive scalar field $\phi(x)$ of charge $q_{e}$ in four dimensions, which is minimally coupled to the gauge field. The wave equation reads as

$$
\frac{1}{\sqrt{-g}} D_{\alpha}\left(\sqrt{-g} g^{\alpha \beta} D_{\beta} \phi(x)\right)+\mu^{2} \phi(x)=0,
$$

where the derivative is defined as $D_{\alpha}=\partial_{\alpha}-i q_{e} A_{\alpha}$. This wave equation is reproduced from a five-dimensional scalar field $\phi_{(5 d)}(x, \chi)$ probing the five-dimensional metric (2), if one takes

$$
\phi_{(5 d)}(x, \chi)=\phi(x) e^{i q_{e} \chi},
$$

and if the five-dimensional mass is equal to $\mu_{(5 d)}^{2}=\mu^{2}+q_{e}^{2}$. However, the five-dimensional scalar is multivalued on the circle $\chi$ unless

$$
q_{e} R_{\chi} \in \mathbb{N} .
$$

This toy model illustrates that the scale $R_{\chi}$ can be constrained from consistent couplings with matter. We will use this quantization condition in Section 6.4. 


\section{Extremal Black Holes as Isolated Systems}

In this section, we review some key properties of extremal black holes in the context of fourdimensional theories of gravity coupled to matter. In one glance, we show that the near-horizon regions of extremal black holes are isolated geometries, isolated thermodynamical systems and, more generally, isolated dynamical systems. We first contrast how to decouple from the asymptotic region the near-horizon region of static and rotating black holes. We then derive the thermodynamic properties of black holes at extremality. We finally discuss uniqueness of near-horizon geometries and their lack of local bulk dynamics.

\subsection{Properties of extremal black holes}

For simplicity, we will strictly concentrate our analysis on stationary black holes. Since the Kerr/CFT correspondence and its extensions are only concerned with the region close to the horizon, one could only require that the near-horizon region is stationary, while radiation would be allowed far enough from the horizon. Such a situation could be treated in the framework of isolated horizons $[11,10]$ (see [14] for a review). However, for our purposes, it will be sufficient and much simpler to assume stationarity everywhere. We expect that all results derived in this review could be generalized for isolated horizons (see [268] for results along these lines).

Many theorems have been derived that characterize the generic properties of four-dimensional stationary black holes that admit an asymptotically-timelike Killing vector. First, they have one additional axial Killing vector - they are axisymmetric ${ }^{3}$ - and their event horizon is a Killing horizon ${ }^{4}$. In asymptotically-flat spacetimes, black holes have spherical topology [163].

Extremal black holes are defined as stationary black holes with vanishing Hawking temperature,

$$
T_{H}=0 .
$$

Equivalently, extremal black holes are defined as stationary black holes whose inner and outer horizons coincide.

No physical process is known that would make an extremal black hole out of a non-extremal black hole. ${ }^{5}$ If one attempts to send finely-tuned particles or waves into a near-extremal black hole in order to further approach extremality, one realizes that there is a smaller and smaller window of parameters that allows one to do so when approaching extremality. In effect, a near-extremal black hole has a potential barrier close to the horizon, which prevents it from reaching extremality. Also, if one artificially continues the parameters of the black holes beyond the extremality bound in a given solution, one typically obtains a naked singularity instead of a black hole. Such naked singularities are thought not to be reachable, which is known as the cosmic censorship hypothesis. Extremal black holes can then be thought of as asymptotic or limiting black holes of physical black holes. The other way around, if one starts with an extremal black hole, one can simply throw in a massive particle to make the black hole non-extremal. Therefore, extremal black holes are finely tuned black holes. Nevertheless, as we will discuss, studying the extremal limit is very interesting because many simplifications occur and powerful specialized methods can be used.

Extremal spinning or charged rotating black holes enjoy several interesting properties that we will summarize below. In order to be self-contained, we will also first provide some properties

3 That has been proven for any non-extremal black hole in $d=4$ Einstein gravity coupled to any matter obeying the weak energy condition with hyperbolic equations of motion and asymptotically-flat boundary conditions [161, 163, 254, 86, 143]. The proof has been extended to extremal black holes, to higher dimensions and to anti-de Sitter asymptotics in $[171,170,85]$.

4 The original proofs were limited to non-extremal black holes, which have a bifurcation surface [66, 163]. The proof for extremal black holes can now be found in [170].

${ }^{5}$ Nevertheless, one can describe the process of spontaneous creation of extremal black holes in an electromagnetic field as an analogue to the Schwinger process of particle creation [126].

Living Reviews in Relativity

http://www. livingreviews . org/Irr-2012-11 
of generic (extremal or non-extremal) black holes. We refer the reader to the excellent lecture notes [259] for the derivation of most of these properties.

- Angular velocity. Spinning black holes are characterized by a chemical potential - the angular velocity $\Omega_{J}$ - conjugate to the angular momentum. The angular velocity can be defined in geometrical terms as the coefficient of the black-hole-horizon generator proportional to the axial Killing vector

$$
\xi=\partial_{t}+\Omega_{J} \partial_{\phi} .
$$

The net effect of the angular velocity is a frame-dragging effect around the black hole. This gravitational kinematics might be the clue of an underlying microscopic dynamics. Part of the intuition behind the extremal spinning black hole/CFT correspondence is that the degrees of freedom responsible for the black hole entropy are rotating at the speed of light at the horizon.

- Electrostatic potential. Electrically-charged black holes are characterized by a chemical potential - the electrostatic potential $\Phi_{e}$ - conjugated to the electric charge. It is defined on the horizon $r=r_{+}$as

$$
\Phi_{e}^{I}=-\left.\xi^{\mu} A_{\mu}^{I}\right|_{r=r_{+}},
$$

where $\xi$ is the horizon generator defined in (9). Similarly, one can associate a magnetic potential $\Phi_{m}^{I}$ to the magnetic monopole charge. The form of the magnetic potential can be obtained by electromagnetic duality, or reads as the explicit formula derived in [99] (see also [91] for a covariant expression). Part of the intuition behind the extremal charged black hole/CFT correspondence is that this kinematics is the sign of microscopic degrees of freedom "moving along the gauge direction". We will make that statement more precise in Section 4.1.

- Ergoregion. Although the Killing generator associated with the mass of the black hole, $\partial_{t}$, is timelike at infinity, it does not need to be timelike everywhere outside the horizon. The region where $\partial_{t}$ is spacelike is called the ergoregion and the boundary of that region where $\partial_{t}$ is lightlike is the ergosphere. If there is no ergoregion, $\partial_{t}$ is a global timelike Killing vector outside the horizon. However, it should be noted that the presence of an ergoregion does not preclude the existence of a global timelike Killing vector. For example, the extremal spinning Kerr-AdS black hole has an ergoregion. When the horizon radius is smaller than the AdS length, the horizon generator becomes spacelike at large enough distances and there is no global timelike Killing vector, as for the Kerr black hole. On the contrary, when the horizon radius is larger than the AdS length, the horizon generator is timelike everywhere outside the horizon and is therefore a global timelike Killing vector.

- Superradiance. One of the most fascinating properties of some rotating black holes is that neutral particles or waves sent towards the black hole with a frequency $\omega$ and angular momentum $m$ inside a specific band

$$
0<\omega<m \Omega_{J}
$$

come back to the exterior region with a higher amplitude. This amplification effect or Penrose effect allows the extraction of energy very efficiently from the black hole. Superradiance occurs for the Kerr and Kerr-Newman black hole and is related to the presence of the ergoregion and the lack of a global timelike Killing vector. Because of the presence of a global timelike Killing vector, there is no superradiance for large Kerr-AdS black holes (when reflective boundary conditions for incident massless waves are imposed) [165, 264].

- Electromagnetic analogue to superradiance. Charged black holes contain electrostatic energy that can also be extracted by sending charged particles or waves with frequency $\omega$ and charge 
$q_{e}$ inside a specific band [84] (see [177] for a review)

$$
0<\omega<q_{e} \Phi_{e}
$$

There is no ergoregion in the four-dimensional spacetime. However, for asymptotically-flat black holes, there is a five-dimensional ergoregion when considering the uplift (2). For the Reissner-Nordström black hole, the five-dimensional ergoregion lies in the range $r_{+}<r<$ $2 M$, where $M$ is the mass and $r$ the standard Boyer-Lindquist radius.

The combined effect of rotation and charge allows one to extract energy in the range

$$
0<\omega<m \Omega_{J}+q_{e} \Phi_{e}
$$

When considering a wave scattering off a black hole, one can define the absorption probability $\sigma_{\text {abs }}$ or macroscopic greybody factor as the ratio between the absorbed flux of energy at the horizon and the incoming flux of energy from infinity,

$$
\sigma_{\mathrm{abs}}=\frac{d E_{\mathrm{abs}} / d t}{d E_{\mathrm{in}} / d t} .
$$

In the superradiant range (13), the absorption probability is negative because the outgoing flux of energy is higher than the incoming flux.

- No thermal radiation but spontaneous emission. Taking quantum mechanical effects into account, non-extremal black holes radiate with a perfect black-body spectrum at the horizon at the Hawking temperature $T_{H}$ [162]. The decay rate of a black hole as observed from the asymptotic region is the product of the black-body spectrum decay rate with the greybody factor $\sigma_{\mathrm{abs}}$,

$$
\Gamma=\frac{1}{e^{\frac{\omega-m \Omega_{J}-q_{e} \Phi_{e}}{T_{H}}}-1} \sigma_{\mathrm{abs}} .
$$

The greybody factor accounts for the fact that waves (of frequency $\omega$, angular momentum $m$ and electric charge $q_{e}$ ) need to travel from the horizon to the asymptotic region in the curved geometry. In the extremal limit, the thermal factor becomes a step function. The decay rate then becomes

$$
\Gamma_{\mathrm{ext}}=-\Theta\left(-\omega+m \Omega_{J}+q_{e} \Phi_{e}\right) \sigma_{\mathrm{abs}} .
$$

As a consequence, ordinary Hawking emission with $\sigma_{\text {abs }}>0$ and $\omega>m \Omega_{J}+q_{e} \Phi_{e}$ vanishes while quantum superradiant emission persists. Therefore, extremal black holes that exhibit superradiance, spontaneously decay to non-extremal black holes by emitting superradiant waves.

- Innermost stable orbit approaching the horizon in the extremal limit. Near-extremal black holes have an innermost stable circular orbit (ISCO) very close to the horizon. (In BoyerLindquist coordinates, the radius of such an orbit coincides with the radius of the horizon. However, since the horizon is a null surface, while the ISCO is timelike, the orbit necessarily lies outside the horizon, which can be seen explicitly in more appropriate coordinates. See Figure 2 of $\left.[34]^{6}\right)$. As a consequence, the region of the black hole close to the horizon can support accretion disks of matter and, therefore, measurements of electromagnetic waves originating from the accretion disk of near-extremal rotating black holes contain (at least some marginal) information from the near-horizon region. For a careful analysis of the physical processes around rotating black holes, see [34]. See also [154] for a recent discussion.

${ }^{6}$ We thank the anonymous referee for pointing out this reference. 
- Classical singularities approaching the horizon in the extremal limit. Stationary axisymmetric non-extremal black holes admit a smooth inner and outer horizon, where curvatures are small. However, numerical results $[52,50,51,112]$ and the identification of unstable linear modes using perturbation theory $[220,125,124]$ showed that the inner horizon is unstable and develops a curvature singularity when the black hole is slightly perturbed. The instability is triggered by tiny bits of gravitational radiation that are blueshifted at the inner Cauchy horizon and which create a null singularity. In the near-extremality limit, the inner horizon approaches the outer horizon and it can be argued that test particles encounter a curvature singularity immediately after they enter the horizon of a near-extremal black hole [212].

\subsection{Near-horizon geometries of static extremal black holes}

As a warm-up, let us first review the near-horizon limit of static extremal black holes. In that case, the generator of the horizon (located at $r=r_{+}$) is the generator of time translations $\partial_{t}$ and the geometry has $S O(3)$ rotational symmetry. Since the horizon generator is null at the horizon, the coordinate $t$ diverges there. The near-horizon limit is then defined as

$$
\begin{array}{r}
t \rightarrow \frac{r_{0} t}{\lambda}, \\
r \rightarrow r_{+}+\lambda r_{0} r,
\end{array}
$$

with $\lambda \rightarrow 0$. The scale $r_{0}$ is introduced for convenience in order to factor out the overall scale of the near-horizon geometry. In the presence of electrostatic potentials, a change of gauge is required when taking the near-horizon limit (17). Indeed, in the near-horizon coordinates (17) the gauge fields take the following form,

$$
A^{I}=-\frac{\Phi_{e}^{I}}{\lambda} r_{0} d t+A_{r}^{I} d r+A_{\theta}^{I} d \theta+A_{\phi}^{I} d \phi
$$

where $\Phi_{e}^{I}$ is the static electric potential of the gauge field $A^{I}$. Upon taking the near-horizon limit one should, therefore, perform a gauge transformation $A^{I} \rightarrow A^{I}+d \Lambda^{I}$ of parameter

$$
\Lambda^{I}=\frac{\Phi_{e}^{I, \text { ext }}}{\lambda} r_{0} t,
$$

where $\Phi_{e}^{I, \text { ext }}$ is the static electric potential at extremality.

It is important to note that one is free to redefine the near-horizon limit parameter $\lambda$ as $\lambda \rightarrow \alpha \lambda$ for any $\alpha>0$. This transformation scales $r$ inversely proportionally to $t$. Therefore, the nearhorizon geometry admits the enhanced symmetry generator

$$
\zeta_{0}=r \partial_{r}-t \partial_{t}
$$

in addition to $\zeta_{-1}=\partial_{t}$ and the $S O(3)$ symmetry generators. Using the properties of static horizons, one can further derive an additional symmetry generator at the horizon $\zeta_{1}$, which together with $\zeta_{-1}$ and $\zeta_{0}$ forms a $S L(2, \mathbb{R})$ algebra. This argument is purely kinematical and does not involve the field equations; see, e.g., [194] for a detailed derivation. The general near-horizon solution compatible with an $S L(2, \mathbb{R}) \times S O(3)$ symmetry is then given by

$$
\begin{array}{r}
d s^{2}=v_{1}\left(-r^{2} d t^{2}+\frac{d r^{2}}{r^{2}}\right)+v_{2}\left(d \theta^{2}+\sin ^{2} \theta d \phi^{2}\right), \\
\chi^{A}=\chi_{\star}^{A}, \quad A^{I}=e_{I} r d t-\frac{p^{I}}{4 \pi} \cos \theta d \phi,
\end{array}
$$


where $v_{1}, v_{2}, \chi_{\star}^{A}, e_{I}, p^{I}$ are parameters, which are constrained by the equations of motion. The geometry consists of the direct product $\mathrm{AdS}_{2} \times S^{2}$.

For some supersymmetric theories, the values $v_{1}, v_{2}, \chi_{\star}^{A}, e_{I}$ are generically completely fixed by the electric $\left(q^{I}\right)$ and magnetic $\left(p^{I}\right)$ charges of the black hole and do not depend continuously on the asymptotic value of the scalar fields in the asymptotic region - the scalar moduli. This is the attractor mechanism [141, 250, 140]. It was then realized that it still applies in the presence of certain higher-derivative corrections [199, 200, 198]. The attractor mechanism was also extended to non-supersymmetric extremal static black holes [139, 240, 150, 179]. As a consequence of this mechanism, the entropy of these extremal black hole does not depend continuously on any moduli of the theory. ${ }^{7}$ The index that captures the entropy can still have discrete jumps when crossing walls of marginal stability in the scalar moduli space [227, 118]. This allows one to account for their black-hole entropy by varying the moduli to a weakly-coupled description of the system without gravity, where states with fixed conserved charges can be counted. Therefore, the attractor mechanism led to an explanation $[18,111]$ of the success of previous string theory calculations of the entropy of certain nonsupersymmetric extremal black holes [181, 172, 110, 260, 131, 132].

As will turn out to be useful in the development of the Reissner-Nordström correspondence, let us discuss the near-horizon geometry (21) under the assumption that one gauge field $A$ can be lifted as a Kaluza-Klein vector to a higher-dimensional spacetime, as discussed in Section 1.2. In the simple model (2), the change of gauge $A \rightarrow A+d \Lambda$ is implemented as the change of coordinates $\chi \rightarrow \chi+\Lambda$. Using the definition of the electrostatic potential $\Phi_{e}^{\text {ext }}(10)$ at extremality, it is straightforward to obtain that in the geometry (2) the horizon is generated by the vector field $\xi_{\text {tot }}=\partial_{t}+\Phi_{e}^{\text {ext }} \partial_{\chi}$. The change of coordinates (17) combined with $\chi \rightarrow \chi+\Lambda$ with $\Lambda$ defined in (19) then maps this vector to

$$
\xi_{\text {tot }} \rightarrow \frac{\lambda}{r_{0}} \partial_{t}
$$

\subsection{Near-horizon of extremal spinning geometries}

Let us now consider extremal spinning black holes. Let us denote the axis of rotation to be $\partial_{\phi}$, where $\phi \sim \phi+2 \pi$ and let $r=r_{+}$be the black-hole horizon. The generator of the horizon is $\xi \equiv \partial_{t}+\Omega_{J}^{\text {ext }} \partial_{\phi}$ where $\Omega_{J}^{\text {ext }}$ is the extremal angular velocity. We choose a coordinate system such that the coordinate $t$ diverges at the horizon, which is equivalent to the fact that $g^{t t}$ diverges at the horizon. As in the static case, one needs to perform a gauge transformation of parameter (19), when electrostatic fields are present. One can again interpret this change of gauge parameter as a change of coordinates in a higher-dimensional auxiliary spacetime (2). The near-horizon limit is then defined as

$$
\begin{array}{r}
t \rightarrow r_{0} \frac{t}{\lambda}, \\
r \rightarrow r_{+}+\lambda r_{0} r, \\
\phi \rightarrow \phi+\Omega_{J}^{\operatorname{ext}} \frac{r_{0} t}{\lambda}, \\
A \rightarrow A+\frac{\Phi_{e}^{\operatorname{ext}}}{\lambda} r_{0} d t,
\end{array}
$$

with $\lambda \rightarrow 0$. The scale $r_{0}$ is again introduced in order to factor out the overall scale of the nearhorizon geometry. The additional effect with respect to the static near-horizon limit is the shift in the angle $\phi$ in order to reach the frame co-moving with the horizon. The horizon generator becomes $\xi=\lambda / r_{0} \partial_{t}$ in the new coordinates. Including the gauge field, one has precisely the relation (22).

\footnotetext{
${ }^{7}$ In some special cases, there may be some continuous dependence of the near-horizon parameters on the scalar moduli, but the entropy is constant under such continuous changes [17].
} 
As in the static case, any finite energy excitation of the near-horizon geometry is confined and amounts to no net charges in the original (asymptotically flat of AdS) geometry.

One is free to redefine $\lambda$ as $\lambda \rightarrow \alpha \lambda$ for any $\alpha>0$ and, therefore, the near-horizon geometry admits the enhanced symmetry generator

$$
\zeta_{0}=r \partial_{r}-t \partial_{t}
$$

in addition to $\zeta_{-1}=\partial_{t}$ and $L_{0}=\partial_{\phi}$. Together $\zeta_{0}$ and $\zeta_{-1}$ form a non-commutative algebra under the Lie bracket.

Now, contrary to the static case, the existence of a third Killing vector is not guaranteed by geometric considerations. Nevertheless, it turns out that Einstein's equations derived from the action (1) imply that there is an additional Killing vector $\zeta_{1}$ in the near-horizon geometry [194, 19] (see also [64] for a geometrical derivation). The vectors $\zeta_{-1}, \zeta_{0}, \zeta_{1}$ turn out to obey the $S L(2, \mathbb{R}) \sim$ $S O(2,1)$ algebra. This dynamical enhancement is at the origin of many simplifications in the near-horizon limit. More precisely, one can prove [194] that any stationary and axisymmetric asymptotically-flat or anti-de Sitter extremal black-hole solution of the theory described by the Lagrangian (1) admits a near-horizon geometry with $S L(2, \mathbb{R}) \times U(1)$ isometry. The result also holds in the presence of higher-derivative corrections in the Lagrangian provided that the black hole is big, in the technical sense that the curvature at the horizon remains finite in the limit where the higher-derivative corrections vanish. The general near-horizon geometry of extremal spinning black holes consistent with these symmetries is given by

$$
\begin{gathered}
d s^{2}=\Gamma(\theta)\left[-r^{2} d t^{2}+\frac{d r^{2}}{r^{2}}+\alpha(\theta)^{2} d \theta^{2}+\gamma(\theta)^{2}(d \phi+k r d t)^{2}\right], \\
\chi^{A}=\chi^{A}(\theta), \quad A^{I}=f^{I}(\theta)(d \phi+k r d t)-\frac{e_{I}}{k} d \phi,
\end{gathered}
$$

where $\Gamma(\theta)>0, \gamma(\theta) \geq 0, \chi^{A}(\theta), f^{I}(\theta)$ and $k, e_{I} \in \mathbb{R}$ are fixed by the equations of motion. By inverting $t$ and redefining $A^{I} \rightarrow-A^{I}$, we can always set $k \geq 0, e_{I} \geq 0$. The function $\alpha(\theta) \geq 0$ can be removed by redefining $\theta$ but it is left for convenience because some near-horizon geometries are then more easily described. ${ }^{8}$

The term $-\frac{e_{I}}{k} d \phi$ in (25) is physical since it cannot be gauged away by an allowed gauge transformation. For example, one can check that the near-horizon energy $\mathcal{Q}_{\partial_{t}}$ would be infinite in the Kerr-Newman near-horizon geometry if this term would be omitted. One can alternatively redefine $f^{I}(\theta)=b^{I}(\theta)+e_{I} / k$ and the gauge field takes the form

$$
A^{I}=b^{I}(\theta)(d \phi+k r d t)+e_{I} r d t .
$$

The static near-horizon geometry (21) is recovered upon choosing only $\mathrm{SO}(3)$ covariant quantities with a well-defined static limit. This requires $k \rightarrow 0$ and it requires the form

$$
b^{I}(\theta)=-\frac{p^{I}}{4 \pi} \cos \theta
$$

where $p^{I}$ are some pure numbers, which are the magnetic charges.

\footnotetext{
${ }^{8}$ We fix the range of $\theta$ as $\theta \in[0, \pi]$. Since the original black hole has $S^{2}$ topology and no conical singularities, the functions $\gamma(\theta), \alpha(\theta)$ also obey regularity conditions at the north and south poles

$$
\frac{\gamma(\theta)^{2}}{\alpha(\theta)^{2}} \sim \theta^{2}+O\left(\theta^{3}\right) \sim(\pi-\theta)^{2}+O\left((\pi-\theta)^{3}\right) .
$$

Similar regularity requirements apply for the scalar and gauge fields.
} 
Going back to the spinning case, the $S L(2, \mathbb{R}) \times U(1)$ symmetry is generated by

$$
\begin{aligned}
& \zeta_{-1}=\partial_{t}, \quad \zeta_{0}=t \partial_{t}-r \partial_{r}, \\
& \zeta_{1}=\left(\frac{1}{2 r^{2}}+\frac{t^{2}}{2}\right) \partial_{t}-t r \partial_{r}-\frac{k}{r} \partial_{\phi}, \quad L_{0}=\partial_{\phi} .
\end{aligned}
$$

In addition, the generator $\zeta_{1}$ should be accompanied by the gauge transformation of parameter $\Lambda^{I}=-e_{I} / r$ so that $\mathcal{L}_{\zeta_{1}} A_{\mu}^{I}+\partial_{\mu} \Lambda^{I}=0$. Note that all of these symmetries act within a threedimensional slice of fixed polar angle $\theta$. The metric is also invariant under discrete symmetry, which maps

$$
(t, \phi) \rightarrow(-t,-\phi)
$$

This is often called the $t$ - $\phi$ reflection symmetry in black-hole literature. The parity/time reversal transformation (30) reverses the electromagnetic charges of the solution.

The geometry (25) is a warped and twisted product of $\mathrm{AdS}_{2} \times S^{2}$. The $(r, t)$ coordinates are analogous to Poincaré coordinates on $\mathrm{AdS}_{2}$ with an horizon at $r=0$. One can find global coordinates in the same way that the global coordinates of $\mathrm{AdS}_{2}$ are related to the Poincaré coordinates [33]. Let

$$
r=\left(1+y^{2}\right)^{1 / 2} \cos \tau+y, \quad t r=\left(1+y^{2}\right)^{1 / 2} \sin \tau
$$

The new axial angle coordinate $\varphi$ is chosen so that $d \phi+k r d t=d \varphi+k y d \tau$, with the result

$$
\phi=\varphi+k \log \left|\frac{\cos \tau+y \sin \tau}{1+\left(1+y^{2}\right)^{1 / 2} \sin \tau}\right| .
$$

In these new coordinates, the near-horizon geometry becomes

$$
\begin{gathered}
d s^{2}=\Gamma(\theta)\left[-\left(1+y^{2}\right) d \tau^{2}+\frac{d y^{2}}{1+y^{2}}+\alpha(\theta)^{2} d \theta^{2}+\gamma(\theta)^{2}(d \varphi+k y d \tau)^{2}\right] \\
\chi^{A}=\chi^{A}(\theta), \quad A^{I}=f^{I}(\theta)(d \varphi+k y d \tau)-\frac{e_{I}}{k} d \varphi
\end{gathered}
$$

after performing an allowed gauge transformation (as the change of gauge falls into the boundary conditions (115) derived in Section 4.1). Note that the $\tau=0$ hypersurface coincides with the $t=0$ hypersurface, and that $\phi=\varphi$ on this hypersurface. The geometry has two boundaries at $y=-\infty$ and $y=+\infty$.

Geodesic completeness of these geometries has not been shown in general, even though it is expected that they are geodesically complete. For the case of the near-horizon geometry of Kerr, geodesic completeness has been proven explicitly in [33] after working out the geodesic equations.

At fixed polar angle $\theta$, the geometry can be described in terms of $3 d$ warped anti-de Sitter geometries; see [8] for a relevant description and [226, 158, 238, 127, 223, 175, 174, 6, 119, 43, 26, 93, 222] for earlier work on these three-dimensional geometries. Warped anti-de Sitter spacetimes are deformations of $\mathrm{AdS}_{3}$, where the $S^{1}$ fiber is twisted around the $\mathrm{AdS}_{2}$ base. Because of the identification $\phi \sim \phi+2 \pi$, the geometries at fixed $\theta$ are quotients of the warped AdS geometries, which are characterized by the presence of a Killing vector of constant norm (namely $\partial_{\phi}$ ). These quotients are often called self-dual orbifolds by analogy to similar quotients in $\mathrm{AdS}_{3}[100]{ }^{9}$

${ }^{9}$ In singular limits where both the temperature and horizon area of black holes can be tuned to zero, while keeping the area-over-temperature-ratio fixed, singular near-horizon geometries can be constructed. Such singular near-horizon geometries contain a local $\mathrm{AdS}_{3}$ factor, which can be either a null self-dual orbifold or a pinching orbifold, as noted in [33, 29, 135, 23] (see [116] for a comprehensive study of the simplest three-dimensional model and [243] for a partial classification of four-dimensional vanishing area near-horizon solutions of (1)). 

if

The geometries enjoy a global timelike Killing vector (which can be identified as $\partial_{\tau}$ ) if and only

$$
k \gamma(\theta)<1, \quad \forall \theta \in[0, \pi] .
$$

If there is no global timelike Killing vector, there is at least one special value of the polar angle $\theta_{\star}$, where $k \gamma\left(\theta_{\star}\right)=1$. At that special value, the slice $\theta=\theta_{\star}$ is locally an ordinary $\operatorname{AdS}_{3}$ spacetime and acquires a local $S L(2, \mathbb{R}) \times S L(2, \mathbb{R})$ isometry. At all other values of $\theta$, one $S L(2, \mathbb{R})$ is broken to $U(1)$. Note that there is still a global time function for each near-horizon geometry. Constant global time $\tau$ in the global coordinates (33) are spacelike surfaces because their normal is timelike,

$$
g^{a b} \partial_{a} \tau \partial_{b} \tau=g^{\tau \tau}=-\left(1+y^{2}\right)^{-1} \Gamma^{-1}(\theta)<0 .
$$

Hence, there are no closed timelike curves.

One can show the existence of an attractor mechanism for extremal spinning black holes, which are solutions of the action (1) [17]. According to [17], the complete near-horizon solution is generically independent of the asymptotic data and depends only on the electric charges $\mathcal{Q}_{e}^{I}$, magnetic charges $\mathcal{Q}_{m}^{I}$ and angular momentum $\mathcal{J}$ carried by the black hole, but in special cases there may be some dependence of the near horizon background on this asymptotic data. In all cases, the entropy only depends on the conserved electromagnetic charges and the angular momentum of the black hole and might only jump discontinuously upon changing the asymptotic values of the scalar fields, as it does for static charged black holes [227, 118].

One can generalize the construction of near-horizon extremal geometries to higher dimensions. In five dimensions, there are two independent planes of rotation since the rotation group is a direct product $S O(4) \sim S O(3) \times S O(3)$. Assuming the presence of two axial $U(1)$ symmetries $\partial_{\phi_{i}}$, $i=1,2$ (with fixed points at the poles), one can prove [194] that the near-horizon geometry of a stationary, extremal black-hole solution of the five-dimensional action (1) possibly supplemented by Chern-Simons terms (4) is given by

$$
\begin{aligned}
& d s^{2}=\Gamma(\theta)\left[-r^{2} d t^{2}+\frac{d r^{2}}{r^{2}}+\alpha(\theta)^{2} d \theta^{2}+\sum_{i, j=1}^{2} \gamma_{i j}(\theta)^{2}\left(d \phi^{i}+k_{i} r d t\right)\left(d \phi^{j}+k_{j} r d t\right)\right] \\
& \chi^{A}=\chi^{A}(\theta), \quad A^{I}=\sum_{i=1}^{2} f_{i}^{I}(\theta)\left(d \phi^{i}+k_{i} r d t\right)-\frac{e_{I}}{k^{i}} d \phi^{i} .
\end{aligned}
$$

In particular, the solutions obtained from the uplift (2) -(3) fall into this class. In general, these solutions can be obtained starting from both black holes (with $S^{3}$ horizon topology) and black rings (with $S^{2} \times S^{2}$ horizon topology) [133].

\subsection{Explicit near-horizon geometries}

Let us now present explicit examples of near-horizon geometries of interest. We will discuss the cases of the extremal Kerr and Reissner-Nordström black holes as well as the extremal KerrNewman and Kerr-Newman-AdS black holes. Other near-horizon geometries of interest can be found, e.g., in [88, 121, 203].

\subsubsection{Near-horizon geometry of extremal Kerr}

The near-horizon geometry of extremal Kerr with angular momentum $\mathcal{J}=J$ can be obtained by the above procedure, starting from the extremal Kerr metric written in usual Boyer-Lindquist coordinates; see the original derivation in [33] as well as in [156, 54]. The result is the NHEK 
geometry, which is written as (25) without matter fields and with

$$
\begin{aligned}
& \alpha(\theta)=1, \quad \Gamma(\theta)=J\left(1+\cos ^{2} \theta\right), \\
& \gamma(\theta)=\frac{2 \sin \theta}{1+\cos ^{2} \theta}, \quad k=1 .
\end{aligned}
$$

The angular momentum only affects the overall scale of the geometry. There is a value $\theta_{\star}=$ $\arcsin (\sqrt{3}-1) \sim 47$ degrees for which $\partial_{t}$ becomes null. For $\theta_{\star}<\theta<\pi-\theta_{\star}, \partial_{t}$ is spacelike. This feature is a consequence of the presence of the ergoregion in the original Kerr geometry. Near the equator we have a "stretched" $\mathrm{AdS}_{3}$ self-dual orbifold (as the $S^{1}$ fiber is streched), while near the poles we have a "squashed" $\mathrm{AdS}_{3}$ self-dual orbifold (as the $S^{1}$ fiber is squashed).

\subsubsection{Near-horizon geometry of extremal Reissner-Nordström}

The extremal Reissner-Nordström black hole is determined by only one parameter: the electric charge $Q$. The mass is $\mathcal{M}=Q$ and the horizon radius is $r_{+}=r_{-}=Q$. This black hole is static and, therefore, its near-horizon geometry takes the form (21). We have explicitly

$$
\nu_{1}=Q^{2}, \quad \nu_{2}=Q^{2}, \quad e=Q, \quad p=0 .
$$

\subsubsection{Near-horizon geometry of extremal Kerr-Newman}

It is useful to collect the different functions characterizing the near-horizon limit of the extremal Kerr-Newman black hole. We use the normalization of the gauge field such that the Lagrangian is proportional to $R-F_{a b} F^{a b}$. The black hole has mass $\mathcal{M}=\sqrt{a^{2}+Q^{2}}$. The horizon radius is given by $r_{+}=r_{-}=\sqrt{a^{2}+Q^{2}}$. One finds

$$
\begin{aligned}
\alpha(\theta) & =1, \quad \Gamma(\theta)=r_{+}^{2}+a^{2} \cos ^{2} \theta, \\
\gamma(\theta) & =\frac{\left(r_{+}^{2}+a^{2}\right) \sin \theta}{r_{+}^{2}+a^{2} \cos ^{2} \theta}, \quad k=\frac{2 a r_{+}}{r_{+}^{2}+a^{2}}, \\
f(\theta) & =Q\left(\frac{r_{+}^{2}+a^{2}}{2 a r_{+}}\right) \frac{r_{+}^{2}-a^{2} \cos ^{2} \theta}{r_{+}^{2}+a^{2} \cos ^{2} \theta}, \quad e=\frac{Q^{3}}{r_{+}^{2}+a^{2}} .
\end{aligned}
$$

In the limit $Q \rightarrow 0$, the NHEK functions (37) are recovered. The near-horizon geometry of extremal Kerr-Newman is therefore smoothly connected to the near-horizon geometry of Kerr. In the limit $a \rightarrow 0$ one finds the near-horizon geometry of the Reissner-Nordström black hole (38). The limiting procedure is again smooth.

\subsubsection{Near-horizon geometry of extremal Kerr-Newman-AdS}

As a last example of near-horizon geometry, let us discuss the extremal spinning charged black hole in AdS or Kerr-Newman-AdS black hole in short. The Lagrangian is given by $L \sim R+6 / l^{2}-F^{2}$ where $l^{2}>0$. It is useful for the following to start by describing a few properties of the nonextremal Kerr-Newman-AdS black hole. The physical mass, angular momentum, electric and magnetic charges at extremality are expressed in terms of the parameters $\left(M, a, Q_{e}, Q_{m}\right)$ of the solution as

$$
\begin{array}{ll}
\mathcal{M}=\frac{M}{\Xi^{2}}, & \mathcal{J}=\frac{a M}{\Xi^{2}}, \\
\mathcal{Q}_{e}=\frac{Q_{e}}{\Xi}, & \mathcal{Q}_{m}=\frac{Q_{m}}{\Xi},
\end{array}
$$


where $\Xi=1-a^{2} / l^{2}$ and $Q^{2}=Q_{e}^{2}+Q_{m}^{2}$. The horizon radius $r_{+}\left(r_{-}\right)$is defined as the largest (smallest) root, respectively, of

$$
\Delta_{r}=\left(r^{2}+a^{2}\right)\left(1+r^{2} / l^{2}\right)-2 M r+Q^{2} .
$$

Hence, one can trade the parameter $M$ for $r_{+}$. If one expands $\Delta_{r}$ up to quadratic order around $r_{+}$, one finds

$$
\Delta_{r}=\Delta_{0}\left(r_{+}-r_{\star}\right)\left(r-r_{+}\right)+\Delta_{0}\left(r-r_{+}\right)^{2}+O\left(r-r_{+}\right)^{3},
$$

where $\Delta_{0}$ and $r_{\star}$ are defined by

$$
\begin{aligned}
\Delta_{0} & =1+a^{2} / l^{2}+6 r_{+}^{2} / l^{2}, \\
\Delta_{0}\left(r_{+}-r_{\star}\right) & =r_{+}\left(1+\frac{a^{2}}{l^{2}}+\frac{3 r_{+}^{2}}{l^{2}}-\frac{a^{2}+Q^{2}}{r_{+}^{2}}\right) .
\end{aligned}
$$

In AdS, the parameter $r_{\star}$ obeys $r_{-} \leq r_{\star} \leq r_{+}$, and coincides with $r_{-}$and $r_{+}$only at extremality. In the flat limit $l \rightarrow \infty$, we have $\Delta_{0} \rightarrow 1$ and $r_{\star} \rightarrow r_{-}$. The Hawking temperature is given by

$$
T_{H}=\frac{\Delta_{0}\left(r_{+}-r_{\star}\right)}{4 \pi\left(r_{+}^{2}+a^{2}\right)} .
$$

The extremality condition is then $r_{+}=r_{\star}=r_{-}$or, more explicitly, the following constraint on the four parameters $\left(r_{+}, a, Q_{e}, Q_{m}\right)$,

$$
1+\frac{a^{2}}{l^{2}}+\frac{3 r_{+}^{2}}{l^{2}}-\frac{a^{2}+Q^{2}}{r_{+}^{2}}=0 .
$$

The near-horizon geometry was obtained in $[159,71]$ (except the coefficient $e$ given here). The result is

$$
\begin{aligned}
\Gamma(\theta) & =\frac{\rho_{+}^{2}}{\Delta_{0}}, \quad \alpha(\theta)=\frac{\Delta_{0}^{1 / 2}}{\Delta_{\theta}^{1 / 2}}, \quad k=\frac{2 a r_{+} \Xi}{\Delta_{0}\left(r_{+}^{2}+a^{2}\right)}, \\
\gamma(\theta) & =\frac{\Delta_{\theta}^{1 / 2} \Delta_{0}^{1 / 2}\left(r_{+}^{2}+a^{2}\right) \sin \theta}{\rho_{+}^{2} \Xi}, \quad e=\frac{Q_{e}}{\Delta_{0}} \frac{r_{+}^{2}-a^{2}}{r_{+}^{2}+a^{2}}, \\
f(\theta) & =\frac{\left(r_{+}^{2}+a^{2}\right)\left[Q_{e}\left(r_{+}^{2}-a^{2} \cos ^{2} \theta\right)+2 Q_{m} a r_{+} \cos \theta\right]}{2 \rho_{+}^{2} \Xi a r_{+}},
\end{aligned}
$$

where we defined

$$
\begin{aligned}
\Delta_{\theta} & =1-\frac{a^{2}}{l^{2}} \cos ^{2} \theta, \quad \rho_{+}^{2}=r_{+}^{2}+a^{2} \cos ^{2} \theta, \\
r_{0}^{2} & =\frac{r_{+}^{2}+a^{2}}{\Delta_{0}} .
\end{aligned}
$$

The near-horizon geometry of the extremal Kerr-Newman black hole is recovered in the limit $l \rightarrow \infty$.

\subsection{Entropy}

The classical entropy of any black hole in Einstein gravity coupled to matter fields such as (1) is given by

$$
\mathcal{S}=\frac{1}{4 G_{N} \hbar} \int_{\Sigma} \operatorname{vol}(\Sigma)
$$


where $\Sigma$ is a cross-section of the black-hole horizon and $G_{N}$ is the four-dimensional Newton's constant. In the near-horizon geometry, the horizon is formally located at any value of $r$ as a consequence of the definition (23). Nevertheless, we can move the surface $\Sigma$ to any finite value of $r$ without changing the integral, thanks to the scaling symmetry $\zeta_{0}$ of (29). Evaluating the expression (49), we obtain

$$
\mathcal{S}=\frac{\pi}{2 G_{N} \hbar} \int_{0}^{\pi} d \theta \alpha(\theta) \Gamma(\theta) \gamma(\theta) .
$$

In particular, the entropy of the extremal Kerr black hole is given by

$$
\mathcal{S}=2 \pi \mathcal{J}
$$

In units of $\hbar$ the angular momentum $\mathcal{J}$ is a dimensionless half-integer. The main result [156, 203, 21, 159, 225, 83, 173, 22, 204, 97] of the extremal spinning black hole/CFT correspondence that we will review below is the derivation of the entropy (50) using Cardy's formula (90).

When higher derivative corrections are considered, the entropy does not scale any more like the horizon area. The black-hole entropy at equilibrium can still be defined as the quantity that obeys the first law of black-hole mechanics, where the mass, angular momenta and other extensive quantities are defined with all higher-derivative corrections included. More precisely, the entropy is first defined for non-extremal black holes by integrating the first law, and using properties of non-extremal black holes, such as the existence of a bifurcation surface [262, 176]. The resulting entropy formula is unique and given by

$$
\mathcal{S}=-\frac{2 \pi}{\hbar} \int_{\Sigma} \frac{\delta^{\mathrm{cov}} L}{\delta R_{a b c d}} \epsilon_{a b} \epsilon_{c d} \operatorname{vol}(\Sigma)
$$

where $\epsilon_{a b}$ is the binormal to the horizon, i.e., the volume element of the normal bundle to $\Sigma$. One can define it simply as $\epsilon_{a b}=n_{a} \xi_{b}-\xi_{a} n_{b}$, where $\xi$ is the generator of the horizon and $n$ is an outgoing null normal to the horizon defined by $n^{2}=0$ and $n^{a} \xi_{a}=-1$. Since the Lagrangian is diffeomorphism invariant (possibly up to a boundary term), it can be expressed in terms of the metric, the matter fields and their covariant derivatives, and the Riemann tensor and its derivatives. This operator $\delta^{\mathrm{cov}} / \delta R_{a b c d}$ acts on the Lagrangian while treating the Riemann tensor as if it were an independent field. It is defined as a covariant Euler-Lagrange derivative as

$$
\frac{\delta^{\mathrm{cov}}}{\delta R_{a b c d}}=\sum_{i=0}(-1)^{i} \nabla_{\left(e_{1}\right.} \ldots \nabla_{\left.e_{i}\right)} \frac{\partial}{\partial \nabla_{\left(e_{1}\right.} \ldots \nabla_{\left.e_{i}\right)} R_{a b c d}} .
$$

Moreover, the entropy formula is conserved away from the bifurcation surface along the future horizon as a consequence of the zeroth law of black-hole mechanics [178]. Therefore, one can take the extremal limit of the entropy formula evaluated on the future horizon in order to define entropy at extremality. Quite remarkably, the Iyer-Wald entropy (52) can also be reproduced [20] using Cardy's formula as we will detail below.

In five-dimensional Einstein gravity coupled to matter, the entropy of extremal black holes can be expressed as

$$
\mathcal{S}=\frac{\pi^{2}}{\hbar G_{N}} \int_{0}^{\pi} d \theta \alpha(\theta) \Gamma(\theta) \gamma(\theta),
$$

where $\Gamma(\theta)$ and $\alpha(\theta)$ have been defined in (36) and $\gamma(\theta)^{2}=\operatorname{det}\left(\gamma_{i j}(\theta)^{2}\right)$.

From the attractor mechanism for four-dimensional extremal spinning black holes [17], the entropy at extremality can be expressed as an extremum of the functional

$$
f\left(\Gamma(\theta), \gamma(\theta), f^{I}(\theta), \chi^{A}(\theta), k, e_{I}\right)=\frac{2 \pi}{G_{N} \hbar}\left(k \mathcal{J}+e_{I} \mathcal{Q}^{I}-\int d \theta d \phi \sqrt{-g} \mathcal{L}\right),
$$


where $\mathcal{L}$ is the Lagrangian. The entropy then only depends on the angular momentum $\mathcal{J}$ and the conserved charges $\mathcal{Q}_{e, m}^{I}$,

$$
\mathcal{S}=\mathcal{S}_{\text {ext }}\left(\mathcal{J}, \mathcal{Q}_{e}^{I}, \mathcal{Q}_{m}^{I}\right)
$$

and depend in a discontinuous fashion on the scalar moduli [240]. The result holds for any Lagrangian in the class (1), including higher-derivative corrections, and the result can be generalized straightforwardly to five dimensions.

When quantum effects are taken into account, the entropy formula also gets modified in a nonuniversal way, which depends on the matter present in quantum loops. In Einstein gravity, the main correction to the area law is a logarithmic correction term. The logarithmic corrections to the entropy of extremal rotating black holes can be obtained using the quantum entropy function formalism [241].

\subsection{Temperature and chemical potentials}

Even though the Hawking temperature is zero at extremality, quantum states just outside the horizon are not pure states when one defines the vacuum using the generator of the horizon. Let us review these arguments following $[156,159,83]$. We will drop the index $I$ distinguishing different gauge fields since this detail is irrelevant to the present arguments.

From the expression of the entropy in terms of the charges $\mathcal{S}_{\text {ext }}\left(\mathcal{J}, \mathcal{Q}_{e}, \mathcal{Q}_{m}\right)$, one can define the chemical potentials

$$
\frac{1}{T_{\phi}}=\left(\frac{\partial \mathcal{S}_{\text {ext }}}{\partial \mathcal{J}}\right)_{\mathcal{Q}_{e, m}}, \quad \frac{1}{T_{e}}=\left(\frac{\partial \mathcal{S}_{\text {ext }}}{\partial \mathcal{Q}_{e}}\right)_{\mathcal{J}, \mathcal{Q}_{m}}, \quad \frac{1}{T_{m}}=\left(\frac{\partial \mathcal{S}_{\text {ext }}}{\partial \mathcal{Q}_{m}}\right)_{\mathcal{J}, \mathcal{Q}_{e}} .
$$

Note that electromagnetic charges are quantized, but when the charges are large one can use the continuous thermodynamic limit. These potentials obey the balance equation

$$
\delta \mathcal{S}_{\text {ext }}=\frac{1}{T_{\phi}} \delta \mathcal{J}+\frac{1}{T_{e}} \delta \mathcal{Q}_{e}+\frac{1}{T_{m}} \delta \mathcal{Q}_{m} .
$$

Another way to obtain these potentials is as follows. At extremality, any fluctuation obeys

$$
0=T_{H} \delta \mathcal{S}=\delta \mathcal{M}-\left(\Omega_{J}^{\mathrm{ext}} \delta \mathcal{J}+\Phi_{e}^{\mathrm{ext}} \delta \mathcal{Q}_{e}+\Phi_{m}^{\mathrm{ext}} \delta \mathcal{Q}_{m}\right),
$$

where $\Omega_{J}^{\text {ext }}$ is the angular potential at extremality and $\Phi_{e, m}^{\text {ext }}$ are electric and magnetic potentials at extremality; see Section 2.1 for a review of these concepts.

One can express the first law at extremality (58) as follows: any variation in $\mathcal{J}$ or $\mathcal{Q}_{m, e}$ is accompanied by an energy variation. One can then solve for $\mathcal{M}=\mathcal{M}_{\text {ext }}\left(\mathcal{J}, \mathcal{Q}_{e}, \mathcal{Q}_{m}\right)$. The first law for a non-extremal black hole can be written as

$$
\delta \mathcal{S}=\frac{1}{T_{H}}\left(\delta \mathcal{M}-\left(\Omega_{J} \delta \mathcal{J}+\Phi_{e} \delta \mathcal{Q}_{e}+\Phi_{m} \delta \mathcal{Q}_{m}\right)\right) .
$$

Let us now take the extremal limit using the following ordering. We first take extremal variations with $\delta \mathcal{M}=\delta \mathcal{M}_{\text {ext }}\left(\mathcal{J}, \mathcal{Q}_{e}, \mathcal{Q}_{m}\right)$. Then, we take the extremal limit of the background configuration. We obtain (57) with

$$
\begin{aligned}
T_{\phi} & =\lim _{T_{H} \rightarrow 0} \frac{T_{H}}{\Omega_{J}^{\text {ext }}-\Omega_{J}}=-\left.\frac{\partial T_{H} / \partial r_{+}}{\partial \Omega_{J} / \partial r_{+}}\right|_{r_{+}=r_{\text {ext }}}, \\
T_{e, m} & =\lim _{T_{H} \rightarrow 0} \frac{T_{H}}{\Phi_{e, m}^{\text {ext }}-\Phi_{e, m}}=-\left.\frac{\partial T_{H} / \partial r_{+}}{\partial \Phi_{e, m} / \partial r_{+}}\right|_{r_{+}=r_{\text {ext }}},
\end{aligned}
$$


where the extremal limit can be practically implemented by taking the limit of the horizon radius $r_{+}$to the extremal horizon radius $r_{\text {ext }}$.

The interpretation of these chemical potentials can be made in the context of quantum field theories in curved spacetimes; see [47] for an introduction. The Hartle-Hawking vacuum for a Schwarzschild black hole, restricted to the region outside the horizon, is a density matrix $\rho=$ $e^{-\omega / T_{H}}$ at the Hawking temperature $T_{H}$. For spacetimes that do not admit a global timelike Killing vector, such as the Kerr geometry, the Hartle-Hawking vacuum does not exist, but one can use the generator of the horizon to define positive frequency modes and, therefore, define the vacuum in the region where the generator is timelike (close enough to the horizon). This is known as the Frolov-Thorne vacuum [144] (see also [128]). One can take a suitable limit of the definition of the Frolov-Thorne vacuum to provide a definition of the vacuum state for any spinning or charged extremal black hole.

Quantum fields for non-extremal black holes can be expanded in eigenstates with asymptotic energy $\hat{\omega}$ and angular momentum $\hat{m}$ with $\hat{t}$ and $\hat{\phi}$ dependence as $e^{-i \hat{\omega} \hat{t}+i \hat{m} \hat{\phi}}$. When approaching extremality, one can perform the change of coordinates (23) in order to zoom close to the horizon. By definition, the scalar field $\phi$ in the new coordinate system $x^{a}=(t, \phi, \theta, r)$ reads in terms of the scalar field $\hat{\phi}$ in the asymptotic coordinate system $\hat{x}^{a}=(\hat{t}, \hat{\phi}, \theta, \hat{r})$ as $\phi\left(x^{a}\right)=\hat{\phi}\left(\hat{x}^{a}\right)$. We can then express

$$
e^{-i \omega t+i m \phi}=e^{-i \hat{\omega} \hat{t}+i \hat{m} \phi},
$$

and the near-horizon parameters are

$$
m=\hat{m}, \quad \omega=\frac{\hat{\omega}-m \Omega_{J}}{\lambda}
$$

When no electromagnetic field is present, any finite energy $\omega$ in the near-horizon limit at extremality $\lambda \rightarrow 0$ corresponds to eigenstates with $\hat{\omega}=\hat{m} \Omega_{J}^{\text {ext }}$. When electric fields are present, zooming in on the near-horizon geometry from a near-extremal solution requires one to perform the gauge transformation $A(x) \rightarrow A(x)+d \Lambda(x)$ with gauge parameter given in (19), which will transform the minimally-coupled charged scalar wavefunction by multiplying it by $e^{i q_{e} \Lambda(x)}$. Finite energy excitations in the near-horizon region then require $\hat{\omega}=m \Omega_{J}^{\text {ext }}+q_{e} \Phi_{e}^{\text {ext }}$. Invoking (classical) electromagnetic duality, the magnetic contribution has the same form as the electric contribution. In summary, the general finite-energy extremal excitation has the form

$$
\hat{\omega}=m \Omega_{J}^{\mathrm{ext}}+q_{e} \Phi_{e}^{\mathrm{ext}}+q_{m} \Phi_{m}^{\mathrm{ext}} .
$$

Following Frolov and Thorne, we assume that quantum fields in the non-extremal geometry are populated with the Boltzmann factor

$$
\exp \left(\hbar \frac{\hat{\omega}-\hat{m} \Omega_{J}-\hat{q}_{e} \Phi_{e}-\hat{q}_{m} \Phi_{m}}{T_{H}}\right),
$$

where $\hat{q}_{e, m}$ are the electric and magnetic charge operators. We also assume that modes obey (64) at extremality. Using the definitions (60)-(61), we obtain the non-trivial extremal Boltzmann factor in the extremal and near-horizon limit

$$
\exp \left(-\hbar \frac{m}{T_{\phi}}-\hbar \frac{q_{e}}{T_{e}}-\hbar \frac{q_{m}}{T_{m}}\right),
$$

where the mode number $m$ and charges $q_{e, m}$ in the near-horizon region are equal to the original mode number and charges $\hat{m}, \hat{q}_{e, m}$. This completes the argument that the Frolov-Thorne vacuum is non-trivially populated in the extremal limit.

Living Reviews in Relativity

http://www. livingreviews.org/lrr-2012-11 
Now, as noted in [4], there is a caveat in the previous argument for the Kerr black hole and, as a trivial generalization, for all black holes that do not possess a global timelike Killing vector. For any non-extremal black hole, the horizon-generating Killing field is timelike just outside the horizon. If there is no global timelike Killing vector, this vector field should become null on some surface at some distance away from the horizon. This surface is called the velocity of light surface. For positive-energy matter, this timelike Killing field defines a positive conserved quantity for excitations in the near-horizon region, ruling out instabilities. However, when approaching extremality, it might turn out that the velocity of light surface approaches asymptotically the horizon. In that case, the horizon-generating Killing field of the extreme black hole may not be everywhere timelike. This causes serious difficulties in defining quantum fields directly in the near-horizon geometry [183, 229, 228]. However, (at least classically) dynamical instabilities might appear only if there are actual bulk degrees of freedom in the near-horizon geometries. We will argue that this is not the case in Section 2.9. As a conclusion, extremal Frolov-Thorne temperatures can be formally and uniquely defined as the extremal limit of non-extremal temperatures and chemical potentials. However, the physical interpretation of these quantities is better understood finitely away from extremality.

The condition for having a global timelike Killing vector was spelled out in (34). This condition is violated for the extremal Kerr black hole or for any extremal Kerr-Newman black hole with $a \geq Q / \sqrt{3}$, as can be shown by using the explicit values defined in (2.4). (The extremal KerrNewman near-horizon geometry does possess a global timelike Killing vector when $a<Q / \sqrt{3}$ and the Kerr-Newman-AdS black holes do as well when $4 a^{2} /\left(\Delta_{0} r_{+}^{2}\right)<1$, which is true for large black holes with $r_{+} \gg l$. Nevertheless, there might be other instabilities due to the electric superradiant effect.)

The extremal Frolov-Thorne temperatures should also be directly encoded in the metric (25). More precisely, these quantities should only depend on the metric and matter fields and not on their equations of motion. Indeed, from the derivation (60) - (61), one can derive these quantities from the angular velocity, electromagnetic potentials and surface gravity, which are kinematical quantities. More physically, the Hawking temperature arises from the analysis of free fields on the curved background, and thus depends on the metric but not on the equations of motion that the metric solves. It should also be the case for the extremal Frolov-Thorne temperatures. Using a reasonable ansatz for the general black-hole solution of (1), including possible higher-order corrections, one can derive $[83,20]$ the very simple formula

$$
T_{\phi}=\frac{1}{2 \pi k} .
$$

From similar considerations, it should also be possible to derive a formula for $T_{e}$ in terms of the functions appearing in (25). We propose simply that

$$
T_{e}=\frac{1}{2 \pi e} .
$$

While we do not have a direct proof of the equivalence between (68) and (61), the formula is consistent with the thermodynamics of (AdS)-Kerr-Newman black holes as one can check from the formulae in Section 2.4. It would be interesting to generalize the arguments of $[83,20]$ to prove the equivalence.

Similarly, one can work out the thermodynamics of five-dimensional rotating black holes. Since there are two independent angular momenta $\mathcal{J}_{1}, \mathcal{J}_{2}$, there are also two independent chemical potentials $T_{\phi_{1}}, T_{\phi_{2}}$ associated with the angular momenta. The same arguments lead to

$$
T_{\phi_{1}}=\frac{1}{2 \pi k_{1}}, \quad T_{\phi_{2}}=\frac{1}{2 \pi k_{2}}
$$


where $k_{1}$ and $k_{2}$ are defined in the near-horizon solution (36).

When considering the uplift (2) of the gauge field along a compact direction of length $2 \pi R_{\chi}$, one can use the definition (69) to define the chemical potential associated with the direction $\partial_{\chi}$. Since the circle has a length $2 \pi R_{\chi}$, the extremal Frolov-Thorne temperature is expressed in units of $R_{\chi}$,

$$
T_{\chi} \equiv T_{e} R_{\chi}=\frac{R_{\chi}}{2 \pi e}
$$

where $T_{e}$ is defined in (68).

\section{Temperatures and entropies of specific extremal black holes}

The entropy of the extremal Kerr black hole is $\mathcal{S}_{\text {ext }}=2 \pi J$. Integrating (57) or using the explicit near-horizon geometry and using (67), we find

$$
T_{\phi}=\frac{1}{2 \pi},
$$

and $T_{e}$ is not defined.

The entropy of the extremal Reissner-Nordström black hole is $\mathcal{S}_{\text {ext }}=\pi Q^{2}$. Integrating (57), we obtain

$$
T_{e}=\frac{1}{2 \pi Q},
$$

while $T_{\phi}$ is not defined.

For the electrically-charged Kerr-Newman black hole, the extremal entropy reads as $\mathcal{S}_{\text {ext }}=$ $\pi\left(a^{2}+r_{+}^{2}\right)$. Expressing the entropy in terms of the physical charges $Q=\sqrt{r_{+}^{2}-a^{2}}$ and $J=a r_{+}$, we obtain

$$
\mathcal{S}_{\mathrm{ext}}=\frac{\pi}{2}\left(\frac{4 J^{2}}{\sqrt{Q^{4}+4 J^{2}}-Q^{2}}+\sqrt{Q^{4}+4 J^{2}}-Q^{2}\right) .
$$

Using (57) and re-expressing in terms of the parameters $\left(a, r_{+}\right)$we find

$$
T_{\phi}=\frac{a^{2}+r_{+}^{2}}{4 \pi a r_{+}}, \quad T_{e}=\frac{a^{2}+r_{+}^{2}}{2 \pi\left(r_{+}^{2}-a^{2}\right)^{3 / 2}} .
$$

We can also derive $T_{\phi}$ from (67) and the explicit near-horizon geometry (39). $T_{e}$ is consistent with (68).

For the extremal Kerr-Newman-AdS black hole, the simplest way to obtain the thermodynamics at extremality is to compute (60)-(61). Using the extremality constraint (46), we obtain

$$
T_{\phi}=\frac{\left(a^{2}+r_{+}^{2}\right) \Delta_{0}}{4 \pi a r_{+} \Xi}, \quad T_{e}=\frac{\left(a^{2}+r_{+}^{2}\right) \Delta_{0}}{2 \pi Q_{e}\left(r_{+}^{2}-a^{2}\right)},
$$

where we used the definitions (48). The magnetic potential $T_{m}$ can then be obtained by electromagnetic duality. The expressions coincide with (67)-(68). These quantities reduce to (74) in the limit of no cosmological constant when there is no magnetic charge, $q_{m}=0$. The extremal entropy is given by $\mathcal{S}_{\text {ext }}=\pi\left(r_{+}^{2}+a^{2}\right) / \Xi$.

\subsection{Near-extremal near-horizon geometries}

An important question about near-horizon geometries is the following: how much dynamics of gravity coupled to matter fields is left in a near-horizon limit such as (23)? We will explore in the following Sections 2.8 and 2.9 several aspects of the dynamics in the near-horizon limit. In 
this section, we will discuss the existence of near-extremal solutions obtained from a combined near-horizon limit and zero temperature limit. We will discuss in Section 2.8 the absence of nonperturbative solutions in the near-horizon geometries, such as black holes. In Section 2.9, we will argue for the absence of local bulk degrees of freedom, and finally in Section 4.4 we will discuss non-trivial boundary dynamics generated by large diffeomorphisms.

Let us first study infinitesimal perturbations of the near-horizon geometry (25). As a consequence of the change of coordinates and the necessary shift of the gauge field (23), the near-horizon energy $\delta \mathcal{Q}_{\partial_{t}}$ of an infinitesimal perturbation is related to the charge associated with the generator of the horizon $\xi_{\text {tot }} \equiv(\xi, \Lambda)=\left(\partial_{t}+\Omega_{J}^{\text {ext }} \partial_{\phi}, \Phi_{e}^{\text {ext }}\right)$ as follows,

$$
\delta \mathcal{Q}_{\xi_{\text {tot }}}=\frac{\lambda}{r_{0}} \delta \mathcal{Q}_{\partial_{t}}, \quad \lambda \rightarrow 0,
$$

as derived in Sections 2.2 and 2.3. Assuming no magnetic charges for simplicity, the conserved charge $\delta \mathcal{Q}_{\xi_{\text {tot }}}$ is given by $\delta \mathcal{M}-\Omega_{J}^{\mathrm{ext}} \delta \mathcal{J}-\Phi_{e}^{\mathrm{ext}} \delta \mathcal{Q}_{e} \cdot{ }^{10}$ Using the first law of thermodynamics valid for arbitrary (not necessarily stationary) perturbations, the left-hand side of (76) can be expressed as

$$
T_{H} \delta \mathcal{S}_{\text {ext }}=\delta \mathcal{Q}_{\xi_{\text {tot }}}
$$

Any geometry that asymptotes to (25) will have finite near-horizon energy $\mathcal{Q}_{\partial_{t}}$. Indeed, an infinite near-horizon energy would be the sign of infrared divergences in the near-horizon geometry and it would destabilize the geometry. It then follows from (76) - (77) that any infinitesimal perturbation of the near-horizon geometry (25) will correspond to an extremal black-hole solution with vanishing Hawking temperature, at least such that $T_{H}=O(\lambda)$. Common usage refers to black-hole solutions, where $T_{H} \sim \lambda$ as near-extremal black holes. Nevertheless, it should be emphasized that after the exact limit $\lambda \rightarrow 0$ is taken the Hawking temperature of such a solution is exactly zero.

We can obtain a near-extremal near-horizon geometry as follows. Starting from a stationary non-extremal black hole of mass $M$ in Boyer-Lindquist coordinates, we perform the near-horizon scaling limit (23) together with the scaling of the temperature

$$
T_{H} \rightarrow \frac{\lambda}{r_{0}} T^{\text {near-ext }} .
$$

While the form of the general non-extremal solution would be required to perform that limit in detail, all examples so far in the class of theories (1), such as the Kerr-Newman-AdS black hole, lead to the following metric

$$
\begin{aligned}
d s^{2} & =\Gamma(\theta)\left[-r\left(r+4 \pi T^{\text {near-ext }}\right) d t^{2}+\frac{d r^{2}}{r\left(r+4 \pi T^{\text {near-ext }}\right)}+\alpha(\theta) d \theta^{2}+\gamma(\theta)(d \phi+k r d t)^{2}\right], \\
\chi^{A} & =\chi^{A}(\theta), \quad A^{I}=f^{I}(\theta)(d \phi+k r d t)-\frac{e_{I}}{k} d \phi .
\end{aligned}
$$

The near-extremal near-horizon solution (79) is diffeomorphic to the near-horizon geometry in Poincaré coordinates (25). Denoting the finite temperature coordinates by a subscript $T$ and the Poincaré coordinates by a subscript $P$, the change of coordinates reads as $[207,247,4,53]$

$$
\begin{aligned}
t_{P} & =\frac{1}{2}\left(\tau^{+}+\tau^{-}\right), \\
r_{P} & =\frac{2}{\tau^{-}-\tau^{+}}, \\
\phi_{P} & =\phi_{F}-2 \pi T^{\text {near-ext }} k t_{F}+\frac{k}{2} \log \left(\frac{1-\left(\tau^{+}\right)^{2}}{1-\left(\tau^{-}\right)^{2}}\right),
\end{aligned}
$$

10 Our conventions for the infinitesimal charges associated with symmetries is as follows: the energy is $\delta \mathcal{M}=\delta \mathcal{Q}_{\partial_{t}}$, the angular momentum is $\delta \mathcal{J}=\delta \mathcal{Q}_{-\partial_{\phi}}$ and the electric charge is $\delta \mathcal{Q}_{e}=\delta \mathcal{Q}_{-\partial_{\chi}}$. In other words, the electric charge is associated with the gauge parameter $\Lambda=-1$. The first law then reads $T_{H} \delta \mathcal{S}=\delta \mathcal{M}-\Omega_{J} \delta \mathcal{J}-\Phi_{e} \delta \mathcal{Q}_{e}$. 
where

$$
\tau^{ \pm}=\tanh \left[\pi T^{\text {near-ext }} t_{F} \pm \frac{1}{4} \log \left(\frac{r_{F}}{r_{F}+4 \pi T^{\text {near-ext }}}\right)\right] .
$$

Therefore, the classical geometries are equivalent. However, since the diffeomorphism is singular at the boundary $r_{F} \rightarrow \infty$, there is a distinction at the quantum level. Since the asymptotic time in near-extremal geometries (79) is different than in extremal geometries (25), fields will be quantized in a different manner in the two geometries.

Let us now compute the energy of these geometries. Multiplying Eq. (76) by $r_{0} / \lambda$ and using (77) and (78), we get that the energy variation around the near-extremal geometry is given by

$$
\not \mathcal{Q}_{\partial_{t}}=T^{\text {near-ext }} \delta \mathcal{S}_{\text {ext }},
$$

where the extremal entropy $\mathcal{S}_{\text {ext }}$ can be expressed in terms of the near-horizon quantities as (50). We denote the variation by $\not$ to emphasize that the energy is not the exact variation of a quantity unless $T^{\text {near-ext }}$ is constant or $\mathcal{S}_{\text {ext }}$ is fixed (which would then lead to zero energy). Therefore, the charge $\not \mathcal{Q}_{\partial_{t}}$ is a heat term, which does not define a conserved energy. Since our derivation of the formula (84) was rather indirect, we check that it is correct for the Kerr-Newman-AdS family of black holes by computing the energy variation directly using the Lagrangian charges defined in $[36,90,97]$.

\subsection{Uniqueness of stationary near-horizon geometries}

We reviewed in Section 2.3 that for any stationary extremal spinning black hole one can isolate a geometry in the vicinity of the horizon, which has enhanced symmetry and universal properties. We discussed in Section 2.7 that another class of stationary near-horizon geometries can be defined, which are, however, related to the extremal near-horizon geometries via a diffeomorphism. It is natural to ask how unique the stationary near-horizon geometries are.

In the case of Einstein gravity, one can prove that the NHEK (near-horizon extremal Kerr geometry) is the unique (up to diffeomorphisms) regular stationary and axisymmetric solution asymptotic to the NHEK geometry with a smooth horizon [4]. This can be understood as a Birkoff theorem for the NHEK geometry. This can be paraphrased by the statement that there are no black holes "inside" of the NHEK geometry. One can also prove that there is a near-horizon geometry in the class (25), which is the unique (up to diffeomorphisms) near-horizon stationary and axisymmetric solution of AdS-Einstein-Maxwell theory [192, 193, 191]. The assumption of axisymmetry can be further relaxed since stationarity implies axisymmetry [170]. It is then natural to conjecture that any stationary solution of the more general action (1), which asymptotes to a near-horizon geometry of the form (25) is diffeomorphic to it. This conjecture remains to be proven.

\subsection{Absence of bulk dynamics in near-horizon geometries}

In this section, we will review arguments pointing to the absence of local degrees of freedom in the near-horizon geometries (25), following the arguments of [4, 122] for Einstein gravity in the NHEK geometry. The only non-trivial dynamics can be argued to appear at the boundary of the near-horizon geometries due to the action of non-trivial diffeomorphisms. The analysis of these diffeomorphisms will be deferred until Section 4.1.

One usually expects that conserved charges are captured by highly-symmetric solutions. From the theorems presented in Section 2.8, we infer that in (AdS)-Einstein-Maxwell theory there is no candidate non-trivial near-horizon solution charged under the $S L(2, \mathbb{R}) \times U(1)$ symmetry $(\times U(1)$ symmetry when electric charge is present), except for a solution related via a diffeomorphism to the near-horizon geometry. If the conjecture presented in Section 2.8 is correct, there is no non-trivial candidate in the whole theory (1). One can then argue that there will be no solution

Living Reviews in Relativity

http://www. livingreviews . org/Irr-2012-11 
- even non-stationary - with non-zero mass or angular momentum (or electric charge when a Maxwell field is present) above the background near-horizon geometry, except solutions related via a diffeomorphism.

In order to test whether or not there exist any local bulk dynamics in the class of geometries, which asymptote to the near-horizon geometries (25), one can perform a linear analysis and study which modes survive at the non-linear level after backreaction is taken into account. This analysis has been performed with care for the spin 2 field around the NHEK geometry in $[4,122]$ under the assumption that all non-linear solutions have vanishing $S L(2, \mathbb{R}) \times U(1)$ charges (which is justified by the existence of a Birkoff theorem as mentioned in Section 2.8). The conclusion is that there is no linear mode that is the linearization of a non-linear solution. In other words, there is no local spin 2 bulk degree of freedom around the NHEK solution. It would be interesting to investigate if these arguments could be generalized to scalars, gauge fields and gravitons propagating on the general class of near-horizon solutions (25) of the action (1), but such an analysis has not been done at that level of generality.

This lack of dynamics is familiar from the $\mathrm{AdS}_{2} \times S^{2}$ geometry [207], which, as we have seen in Sections 2.2-2.3, is the static limit of the spinning near-horizon geometries. In the above arguments, the presence of the compact $S^{2}$ was crucial. Conversely, in the case of non-compact horizons, such as the extremal planar AdS-Reissner-Nordström black hole, flux can leak out the $\mathbb{R}^{2}$ boundary and the arguments do not generalize straightforwardly. There are indeed interesting quantum critical dynamics around $\mathrm{AdS}_{2} \times \mathbb{R}^{2}$ near-horizon geometries [136], but we will not touch upon this topic here since we concentrate exclusively on compact black holes. 


\section{Two-Dimensional Conformal Field Theories}

Since we aim at drawing parallels between black holes and two-dimensional CFTs (2d CFTs), it is useful to describe some key properties of $2 d$ CFTs. Background material can be found, e.g., in $[120,149,234]$. An important caveat to keep in mind is that there are only sparse results in gravity that can be interpreted in terms of a $2 d$ CFT. Only future research will tell if $2 d$ CFTs are the right theories to be considered (if a holographic correspondence can be precisely formulated at all) or if generalized field theories with conformal invariance are needed. For progress in this direction, see [130, 169].

A $2 d$ CFT is defined as a local quantum field theory with local conformal invariance. In twodimensions, the local conformal group is an infinite-dimensional extension of the globally-defined conformal group $S L(2, \mathbb{R}) \times S L(2, \mathbb{R})$ on the plane or on the cylinder. It is generated by two sets of vector fields $L_{n}, \bar{L}_{n}, n \in \mathbb{Z}$ obeying the Lie bracket algebra

$$
\begin{aligned}
& {\left[L_{m}, L_{n}\right]=(m-n) L_{m+n},} \\
& {\left[L_{m}, \bar{L}_{n}\right]=0,} \\
& {\left[\bar{L}_{m}, \bar{L}_{n}\right]=(m-n) \bar{L}_{m+n} .}
\end{aligned}
$$

From Noether's theorem, each symmetry is associated to a quantum operator. The local conformal symmetry is associated with the conserved and traceless stress-energy tensor operator, which can be decomposed into left and right moving modes $\mathcal{L}_{n}$ and $\overline{\mathcal{L}}_{n}, n \in \mathbb{Z}$. The operators $\mathcal{L}_{n}, \overline{\mathcal{L}}_{n}$ form two copies of the Virasoro algebra

$$
\begin{aligned}
& {\left[\mathcal{L}_{m}, \mathcal{L}_{n}\right]=(m-n) \mathcal{L}_{m+n}+\frac{c_{L}}{12} m\left(m^{2}-A_{L}\right) \delta_{m+n, 0},} \\
& {\left[\mathcal{L}_{m}, \overline{\mathcal{L}}_{n}\right]=0} \\
& {\left[\overline{\mathcal{L}}_{m}, \overline{\mathcal{L}}_{n}\right]=(m-n) \overline{\mathcal{L}}_{m+n}+\frac{c_{R}}{12} m\left(m^{2}-A_{R}\right) \delta_{m+n, 0},}
\end{aligned}
$$

where $\mathcal{L}_{-1}, \mathcal{L}_{0}, \mathcal{L}_{1}\left(\right.$ and $\left.\overline{\mathcal{L}}_{-1}, \overline{\mathcal{L}}_{0}, \overline{\mathcal{L}}_{1}\right)$ span a $S L(2, \mathbb{R})$ subalgebra. The pure numbers $c_{L}$ and $c_{R}$ are the left and right-moving central charges of the CFT. The auxiliary parameters $A_{L}, A_{R}$ depend if the CFT is defined on the plane or on the cylinder. They correspond to shifts of the background value of the zero eigenmodes $\mathcal{L}_{0}, \overline{\mathcal{L}}_{0}$. In many examples of CFTs, additional symmetries are present in addition to the two sets of Virasoro algebras.

A $2 d$ CFT can be uniquely characterized by a list of (primary) operators $\mathcal{O}$, the conformal dimensions of these operators (their eigenvalue under $\mathcal{L}_{0}$ and $\overline{\mathcal{L}}_{0}$ ) and the operator product expansions between all operators. Since we will only be concerned with universal properties of CFTs here, such detailed data of individual CFTs will not be important for our considerations.

We will describe in the next short Sections 3.1, 3.2 and 3.3 some properties of CFTs that are conjectured to be relevant to the Kerr/CFT correspondence and its extensions: the Cardy formula, some properties of the discrete light-cone quantization (DLCQ) and some properties of symmetric product orbifold CFTs.

\subsection{Cardy's formula}

In any unitary and modular invariant CFT, the asymptotic growth of states in the microcanonical ensemble is determined only by the left and right central charge and the left and right eigenvalues $\mathcal{L}_{0}, \overline{\mathcal{L}}_{0}$ as

$$
\mathcal{S}_{\mathrm{CFT}}=2 \pi\left(\sqrt{\frac{c_{L} \mathcal{L}_{0}}{6}}+\sqrt{\frac{c_{R} \overline{\mathcal{L}}_{0}}{6}}\right)
$$


when $\mathcal{L}_{0} \gg c_{L}, \overline{\mathcal{L}}_{0} \gg c_{R}$. This is known as Cardy's formula derived originally in [61, 48] using modular invariance of the CFT. A review can be found, e.g., in [62]. Transforming to the canonical ensemble using the definition of the left and right temperatures,

$$
\left(\frac{\partial \mathcal{S}_{\mathrm{CFT}}}{\partial \mathcal{L}_{0}}\right)_{\overline{\mathcal{L}}_{0}}=\frac{1}{T_{L}}, \quad\left(\frac{\partial \mathcal{S}_{\mathrm{CFT}}}{\partial \overline{\mathcal{L}}_{0}}\right)_{\mathcal{L}_{0}}=\frac{1}{T_{R}},
$$

we get

$$
\mathcal{L}_{0}=\frac{\pi^{2}}{6} c_{L} T_{L}^{2}, \quad \overline{\mathcal{L}}_{0}=\frac{\pi^{2}}{6} c_{R} T_{R}^{2},
$$

and, therefore, we obtain an equivalent form of Cardy's formula,

$$
\mathcal{S}_{\mathrm{CFT}}=\frac{\pi^{2}}{3}\left(c_{L} T_{L}+c_{R} T_{R}\right)
$$

valid when $T_{L} \gg 1, T_{R} \gg 1$.

\subsection{DLCQ and chiral limit of CFTs}

The role of the DLCQ of CFTs in the context of the Kerr/CFT correspondence was suggested in [30] (for closely related work see [252]). Here, we will review how a DLCQ is performed and how it leads to a chiral half of a CFT. A chiral half of a CFT is here defined as a sector of a $2 d$ CFT defined on the cylinder, where the right-movers are set to the ground state after the limiting DLCQ procedure. We will use these considerations in Section 4.4.

Let us start with a CFT defined on a cylinder of radius $R$,

$$
d s^{2}=-d t^{2}+d \phi^{2}=-d u d v, \quad u=t-\phi, \quad v=t+\phi .
$$

Here the coordinates are identified as $(t, \phi) \sim(t, \phi+2 \pi R)$, which amounts to

$$
(u, v) \sim(u-2 \pi R, v+2 \pi R) .
$$

The momentum operators $P^{v}$ and $P^{u}$ along the $u$ and $v$ directions are $L_{0}$ and $\bar{L}_{0}$, respectively. They have a spectrum

$$
\begin{aligned}
& P^{v}|O\rangle=L_{0}|O\rangle=(h+n) \frac{1}{R}|O\rangle, \\
& P^{u}|O\rangle=\bar{L}_{0}|O\rangle=(\bar{h}+\bar{n}) \frac{1}{R}|O\rangle,
\end{aligned}
$$

where the conformal dimensions obey $h, \bar{h} \geq 0$ and $n, \bar{n} \neq 0$ are quantized left and right momenta.

Following Seiberg [239], consider a boost with rapidity $\gamma$

$$
u^{\prime}=e^{\gamma} u, \quad v^{\prime}=e^{-\gamma} v .
$$

The boost leaves the flat metric invariant. The discrete light-cone quantization of the CFT is then defined as the limit $\gamma \rightarrow \infty$ with $R^{\prime} \equiv R e^{\gamma}$ fixed. In that limit, the identification (92) becomes

$$
\left(u^{\prime}, v^{\prime}\right) \sim\left(u^{\prime}-2 \pi R^{\prime}, v^{\prime}\right) .
$$

Therefore, the resulting theory is defined on a null cylinder. Because of the boosted kinematics, we have

$$
\begin{aligned}
& P^{v^{\prime}}|O\rangle=(h+n) \frac{1}{R e^{\gamma}}|O\rangle, \\
& P^{u^{\prime}}|O\rangle=(\bar{h}+\bar{n}) \frac{e^{\gamma}}{R}|O\rangle .
\end{aligned}
$$


Keeping $P^{u^{\prime}}$ (the momentum along $v^{\prime}$ ) finite in the $\gamma \rightarrow \infty$ limit requires $\bar{h}=0$ and $\bar{n}=0$.

Therefore, the DLCQ limit requires one to freeze the right-moving sector to the vacuum state. The resulting theory admits an infinite energy gap in that sector. The left-moving sector still admits non-trivial states. All physical finite-energy states in this limit only carry momentum along the compact null direction $u^{\prime}$. Therefore, the DLCQ limit defines a Hilbert space $\mathcal{H}$,

$$
\left.\mathcal{H}=\{\mid \text { anything }\rangle_{L} \otimes|0\rangle_{R}\right\}
$$

with left chiral excitations around the $S L(2, \mathbb{R}) \times S L(2, \mathbb{R})$ invariant vacuum of the CFT $|0\rangle_{L} \otimes|0\rangle_{R}$. As a consequence, the right-moving Virasoro algebra does not act on that Hilbert space. This is by definition a chiral half of a CFT.

In summary, the DLCQ of a $2 d$ CFT leads to a chiral half of the CFT with central charge $c=c_{L}$. The limiting procedure certainly removes most of the dynamics of the original CFT. How much dynamics is left in a chiral half of a CFT is an important question that is left to be examined in detail in the future.

\subsection{Long strings and symmetric orbifolds}

Given a set of Virasoro generators $\mathcal{L}_{n}$ and a non-zero integer $N \in \mathbb{Z}_{0}$, one can always redefine a subset or an extension of the generators, which results in a different central charge (see, e.g., [25]). One can easily check that the generators

$$
\mathcal{L}_{n}^{\text {short }}=\frac{1}{N} \mathcal{L}_{N n}
$$

obey the Virasoro algebra with a larger central charge $c^{\text {short }}=N c$. Conversely, one might define

$$
\mathcal{L}_{n}^{\text {long }}=N \mathcal{L}_{n / N}
$$

In general, the generators $\mathcal{L}_{n}^{\text {long }}$ with $n \neq N k, k \in \mathbb{Z}$ do not make sense because there are no fractionalized Virasoro generators in the CFT. Such generators would be associated with multivalued modes $e^{i n \phi / N}$ on the cylinder $(t, \phi) \sim(t, \phi+2 \pi)$. However, in some cases, as we review below, the Virasoro algebra (101) can be defined. The resulting central charge is smaller and given by $c^{\text {long }}=c / N$.

If a CFT with generators (101) can be defined such that it still captures the entropy of the original CFT, the Cardy formula (90) applied in the original CFT could then be used outside of the usual Cardy regime $T_{L} \gg 1$. Indeed, using the CFT with left-moving generators (101) and their right-moving analogue, one has

$$
\mathcal{S}_{\mathrm{CFT}}=\frac{\pi^{2}}{3}\left(\frac{c_{L}}{N}\left(N T_{L}\right)+\frac{c_{R}}{N}\left(N T_{R}\right)\right)
$$

which is valid when $N T_{L} \gg 1, N T_{R} \gg 1$. If $N$ is very large, Cardy's formula (90) would then always apply. We will use the assumption of the existence of such a "long string CFT" in Section 4.4 to justify the validity of Cardy's formula outside the usual Cardy regime as done originally in [157].

The "long string CFT" can be made more explicit in the context of symmetric product orbifold CFTs [186], which appear in the $\mathrm{AdS}_{3} / \mathrm{CFT}_{2}$ correspondence [206, 114, 123] (see also [230] and references therein). These orbifold CFTs can be argued to be relevant in the present context, since the Kerr/CFT correspondence might be understood as a deformation of the $\mathrm{AdS}_{3} / \mathrm{CFT}_{2}$ correspondence, as argued in $[157,98,23,116,31,243,246,115,130]$.

Let us then briefly review the construction of symmetric product orbifold CFTs. Given a conformally-invariant sigma-model with target space manifold $\mathcal{M}$, one can construct the symmetric product orbifold by considering the sigma-model with $N$ identical copies of the target space

Living Reviews in Relativity

http://www. livingreviews . org/Irr-2012-11 
manifold $\mathcal{M}$, identified up to permutations,

$$
\operatorname{Sym}^{N}(\mathcal{M}) \equiv\left(\otimes^{N} \mathcal{M}\right) / S_{N}
$$

where $S_{N}$ is the permutation group on $N$ objects. The low energy (infrared) dynamics is a CFT with central charge $c_{S y m}=N c$ if the central charge of the low energy CFT of the original sigma model is $c$. The Virasoro generators of the resulting infrared CFT can then be formally constructed from the generators $\mathcal{L}_{m}$ of the original infrared CFT as (100). Conversely, if one starts with a symmetric product orbifold, one can isolate the "long string" sector, which contains the "long" twisted operators. One can argue that such a sector can be effectively described in the infrared by a CFT, which has a Virasoro algebra expressed as (101) in terms of the Virasoro algebra of the low energy CFT of the symmetric product orbifold [211]. The role of these constructions for the Kerr/CFT correspondence remains to be fully understood. 


\section{Microscopic Entropy of Extremal Black Holes}

We discussed that near-horizon geometries of compact extremal black holes are isolated systems with universal properties and we reviewed that in all analyzed cases they have no local bulk dynamics. Given the non-trivial thermodynamic properties of these systems even at extremality, one can suspect that some non-trivial dynamics are left. It turns out that such non-trivial dynamics appears at the boundary of the near-horizon geometry. We now show that near-horizon geometries can be extended to a large class describing extremal boundary excitations. The set of all nearhorizon geometries will admit additional symmetries at their boundary - asymptotic symmetries which will turn out to be given by one copy of the Virasoro algebra. We will then argue that these near-horizon geometries are described by chiral limits of two-dimensional CFTs, which we will use to microscopically derive the entropy of any charged or spinning extremal black hole.

\subsection{Boundary conditions and asymptotic symmetry algebra}

Let us discuss the existence and the construction of a consistent set of boundary conditions that would define "the set of solutions in the near-horizon region of extremal black holes". Since the near-horizon region is not asymptotically flat or asymptotically anti-de Sitter, one cannot use previous results in those spacetimes to derive the boundary conditions in the near-horizon region. Rather, one has to derive the relevant boundary conditions from first principles. A large literature on the theory of boundary conditions and asymptotic charges exists, see [9, 237, 59, 196, 35, 36] (see also [90] for a review). We will use the Lagrangian methods [35, 36] to address the current problem.

A set of boundary conditions always comes equipped with an asymptotic symmetry algebra. Restricting our discussion to the fields appearing in (1), the boundary conditions are preserved by a set of allowed diffeomorphisms and $U(1)$ gauge transformations $\left(\zeta^{\mu}, \Lambda\right)$, which act on the fields as

$$
\begin{aligned}
\delta_{(\zeta, \Lambda)} g_{\mu \nu} & =\mathcal{L}_{\zeta} g_{\mu \nu}, \quad \delta_{(\zeta, \Lambda)} A_{\mu}=\mathcal{L}_{\zeta} A_{\mu}+\partial_{\mu} \Lambda \\
\delta_{(\zeta, \Lambda)} \chi^{A} & =\mathcal{L}_{\zeta} \chi^{A} .
\end{aligned}
$$

Asymptotic symmetries are the set of all these allowed transformations that are associated with non-trivial conserved charges. The set of allowed transformations that are associated with zero charges are "pure gauge" or "trivial" transformations. The set of asymptotic symmetries inherits a Lie algebra structure from the Lie commutator of diffeomorphisms and $U(1)$ gauge transformations. Therefore, the asymptotic symmetries form an algebra,

$$
\left[\left(\zeta_{m}, \Lambda_{m}\right),\left(\zeta_{n}, \Lambda_{n}\right)\right] \equiv\left(\left[\zeta_{m}, \zeta_{n}\right],\left[\Lambda_{m}, \Lambda_{n}\right]_{\zeta}\right)
$$

where $\left[\zeta_{m}, \zeta_{n}\right]$ is the Lie commutator and

$$
\left[\Lambda_{m}, \Lambda_{n}\right]_{\zeta} \equiv \zeta_{m}^{\mu} \partial_{\mu} \Lambda_{n}-\zeta_{n}^{\mu} \partial_{\mu} \Lambda_{m}
$$

Consistency requires that the charge associated with each element of the asymptotic symmetry algebra be finite and well defined. Moreover, as we are dealing with a spatial boundary, the charges are required to be conserved in time. By construction, one always first defines the "infinitesimal variation of the charge" $\delta \mathcal{Q}$ from infinitesimal variations of the fields around a solution. If $\delta \mathcal{Q}$ is the exact variation of a quantity $\mathcal{Q}$, the quantity $\mathcal{Q}$ is the well-defined charge and the charges are said to be integrable.

Imposing consistent boundary conditions and obtaining the associated asymptotic symmetry algebra requires a careful analysis of the asymptotic dynamics of the theory. If the boundary conditions are too strong, all interesting excitations are ruled out and the asymptotic symmetry

Living Reviews in Relativity

http://www. livingreviews . org/Irr-2012-11 
algebra is trivial. If they are too weak, the boundary conditions are inconsistent because transformations preserving the boundary conditions are associated to infinite or ill-defined charges. In general, there is a narrow window of consistent and interesting boundary conditions. There is not necessarily a unique set of consistent boundary conditions.

There is no universal algorithm to define the boundary conditions and the set of asymptotic symmetries. One standard algorithm used, for example, in $[168,167]$ consists in first promoting all exact symmetries of the background solution as asymptotic symmetries and second acting on solutions of interest with the asymptotic symmetries in order to generate tentative boundary conditions. The boundary conditions are then restricted in order to admit consistent finite, well defined and conserved charges. Finally, the set of asymptotic diffeomorphisms and gauge transformations, which preserve the boundary conditions are computed and one deduces the full asymptotic symmetry algebra after computing the associated conserved charges.

As an illustration, asymptotically anti-de Sitter spacetimes in spacetime dimensions $d+1$ admit the $S O(2, d)$ asymptotic symmetry algebra for $d \geq 3[1,15,168,167]$ and two copies of the Virasoro algebra for $d=2$ [58]. Asymptotically-flat spacetimes admit as asymptotic symmetry algebra the Poincaré algebra or an extension thereof depending on the precise choice of boundary conditions $[9,231,147,237,13,12,16,37,38,92,261]$. From these examples, we learn that the asymptotic symmetry algebra can be larger than the exact symmetry algebra of the background spacetime and it might in some cases contain an infinite number of generators. We also notice that several choices of boundary conditions, motivated from different physical considerations, might lead to different asymptotic symmetry algebras.

Let us now motivate boundary conditions for the near-horizon geometry of extremal black holes. There are two boundaries at $r=\infty$ and $r=-\infty$. It was proposed in $[156,159]$ to build boundary conditions on the boundary $r=\infty$ such that the asymptotic symmetry algebra contains one copy of the Virasoro algebra generated by

$$
\begin{aligned}
\zeta_{\epsilon} & =\epsilon(\phi) \partial_{\phi}-r \epsilon^{\prime}(\phi) \partial_{r}+(\text { subleading terms }), \\
\Lambda_{\epsilon} & =-\left(f(\theta)-\frac{e}{k}\right) \epsilon(\phi)+(\text { subleading terms }) .
\end{aligned}
$$

Part of the physical motivation behind this ansatz is the existence of a non-zero temperature $T_{\phi}$ associated with modes corotating with the black hole, as detailed in Section 2.6. This temperature suggests the existence of excitations along $\partial_{\phi}$. The ansatz for $\Lambda_{\epsilon}$ will be motivated in (117). The subleading terms might be chosen such that the generator $\zeta_{\epsilon}$ is regular at the poles $\theta=0, \pi$. This ansatz has to be validated by checking if boundary conditions preserved by this algebra exist such that all charges are finite, well defined and conserved. We will discuss such boundary conditions below. Expanding in modes as ${ }^{11}$

$$
\epsilon(\phi)=-e^{-i n \phi},
$$

the generators $L_{n} \equiv\left(\zeta_{n}, \Lambda_{n}\right)$ obey the Virasoro algebra with no central extension

$$
i\left[L_{m}, L_{n}\right]=(m-n) L_{m+n},
$$

where the bracket has been defined in (105).

Finding consistent boundary conditions that admit finite, conserved and integrable Virasoro charges and that are preserved by the action of the Virasoro generators is a non-trivial task. The details of these boundary conditions depend on the specific theory at hand because the expression for the conserved charges depend on the theory. (For the action (1), the conserved charges can be found in [97]). Specializing in the case of the extremal Kerr black hole in Einstein gravity, the

11 The sign choice in this expansion is motivated by the fact that the central charge to be derived in Section 4.3 will be positive with this choice. Also, the zero mode $\epsilon=-1$ is canonically associated with the angular momentum in our conventions. 
problem of finding consistent boundary conditions becomes more manageable but is still intricate (see discussions in [5]). In [156], the following fall-off conditions

$$
\begin{array}{rlrl}
g_{t t} & =\mathcal{O}\left(r^{2}\right), & g_{t \phi} & =k \Gamma(\theta) \gamma(\theta)^{2} r+\mathcal{O}(1), \\
g_{t \theta} & =\mathcal{O}\left(\frac{1}{r}\right), & g_{t r}=\mathcal{O}\left(\frac{1}{r^{2}}\right), & g_{\phi \phi}=\mathcal{O}(1), \\
g_{\phi \theta} & =\mathcal{O}\left(\frac{1}{r}\right), & g_{\phi r}=\mathcal{O}\left(\frac{1}{r}\right), & g_{\theta r}=\mathcal{O}\left(\frac{1}{r^{2}}\right), \\
g_{\theta \theta} & =\Gamma(\theta) \alpha(\theta)^{2}+\mathcal{O}\left(\frac{1}{r}\right), \quad g_{r r}=\frac{\Gamma(\theta)}{r^{2}}+\mathcal{O}\left(\frac{1}{r^{3}}\right),
\end{array}
$$

were proposed as a part of the definition of boundary conditions. The zero energy excitation condition

$$
\delta \mathcal{Q}_{\partial_{t}}=0
$$

was imposed as a supplementary condition. We will discuss in Section 4.2 the relaxation of this condition. A non-trivial feature of the boundary conditions (111)-(112) is that they are preserved precisely by the Virasoro algebra (107), by $\partial_{t}$ and the generator (24) (as pointed out in [5]) and subleading generators. (Note that these boundary conditions are not preserved by the action of the third $S L(2, \mathbb{R})$ generator (29).) It was shown in [156] that the Virasoro generators are finite given the fall-off conditions and well defined around the background NHEK geometry. It was shown in [5] that the Virasoro generators are conserved and well defined around any asymptotic solution given that one additionally regularizes the charges using counter-term methods [96]. Therefore, up to some technical details that remain to be fully understood, it can be claimed that consistent boundary conditions admitting (at least) a Virasoro algebra as asymptotic symmetry algebra exist. The set of trivial asymptotic symmetries comprise two of the $S L(2, \mathbb{R})$ generators. It is not clear if the boundary conditions could be enhanced in order to admit all $S L(2, \mathbb{R})$ generators as trivial asymptotic symmetries.

Let us now generalize these arguments to the electrically-charged Kerr-Newman black hole in Einstein-Maxwell theory. First, the presence of the chemical potential $T_{e}$ suggests that some dynamics are also present along the gauge field. The associated conserved electric charge $\mathcal{Q}_{e}$ can be shown to be canonically associated with the zero-mode generator $J_{0}=(0,-1)$ with gauge parameter $\Lambda=-1$. It is then natural to define the current ansatz

$$
J_{n}=\left(0,-e^{-i n \phi}\right),
$$

which obeys the commutation relations

$$
i\left[L_{m}, J_{n}\right]=-n J_{m+n}, \quad i\left[J_{m}, J_{n}\right]=0 .
$$

The non-trivial step consists in establishing the existence of boundary conditions such that the Virasoro and the current charges are well defined and conserved. Ongoing work is in progress in that direction. ${ }^{12}$ One can simplify the problem of constructing boundary conditions by imposing the following additional constraints

$$
\delta \mathcal{Q}_{\partial_{t}}=0, \quad \delta \mathcal{Q}_{e}=0,
$$

which discard the current algebra. Such a simplification was used in [159] and the following boundary conditions were proposed (up to the term $e / k$, which was omitted in [159])

$$
\begin{array}{ll}
A_{t}=\mathcal{O}(r), & A_{\phi}=f(\theta)-\frac{e}{k}+\mathcal{O}\left(\frac{1}{r}\right), \\
A_{\theta}=\mathcal{O}(1), & A_{r}=\mathcal{O}\left(\frac{1}{r^{2}}\right),
\end{array}
$$

12 Compère, in preparation, (2012). 
which are preserved upon acting with the Virasoro generator (107) - (108). In particular, the choice of the compensating gauge transformation $\Lambda_{\epsilon}(108)$ is made such that

$$
\mathcal{L}_{\zeta_{\epsilon}} A_{\phi}+\partial_{\phi} \Lambda_{\epsilon}=O\left(r^{-1}\right) .
$$

It can be shown that the Virasoro generators are finite under these boundary conditions.

Let us also discuss what happens in higher dimensions $(d>4)$. The presence of several independent planes of rotation allows for the construction of one Virasoro ansatz and an associated Frolov-Thorne temperature for each plane of rotation [203, 173, 21, 225, 83]. More precisely, given $n$ compact commuting Killing vectors, one can consider an $S L(n, \mathbb{Z})$ family of Virasoro ansätze by considering all modular transformations on the $U(1)^{n}$ torus [201, 76]. However, preliminary results show that there is no boundary condition that allows simultaneously two different Virasoro algebras in the asymptotic symmetry algebra [21]. Rather, there are mutually-incompatible boundary conditions for each choice of Virasoro ansatz.

Since two $U(1)$ circles form a torus invariant under $S L(2, \mathbb{Z})$ modular transformations, one can then form an ansatz for a Virasoro algebra for any circle defined by a modular transformation of the $\phi_{1}$ and $\phi_{2}$-circles. More precisely, we define

$$
\phi_{1}^{\prime}=p_{1} \phi_{1}+p_{2} \phi_{2}, \quad \phi_{2}^{\prime}=p_{3} \phi_{1}+p_{4} \phi_{2},
$$

where $p_{1} p_{4}-p_{2} p_{3}=1$ and we consider the vector fields

$$
L_{n}^{\left(p_{1}, p_{2}\right)}=-e^{-i n \phi_{1}^{\prime}} \partial_{\phi_{1}^{\prime}}-i r e^{-i n \phi_{1}^{\prime}} \partial_{r}+\text { (subleading terms). }
$$

The resulting boundary conditions have not been thoroughly constructed, but evidence points to their existence [21, 201].

The occurrence of multiple choices of boundary conditions in the presence of multiple $U(1)$ symmetries raises the question of whether or not the (AdS)-Reissner-Nördstrom black hole admits interesting boundary conditions where the $U(1)$ gauge symmetry (which is canonically associated to the conserved electric charge $Q$ ) plays the prominent role. One can also ask these questions for the general class of (AdS)-Kerr-Newman black holes.

It was argued in $[159,204]$ that such boundary conditions indeed exist when the $U(1)$ gauge field can be promoted to be a Kaluza-Klein direction of a higher-dimensional spacetime, or at least when such an effective description captures the physics. Denoting the additional direction by $\partial_{\chi}$ with $\chi \sim \chi+2 \pi R_{\chi}$, the problem amounts to constructing boundary conditions in five dimensions. As mentioned earlier, evidence points to the existence of such boundary conditions [21, 201]. The Virasoro asymptotic-symmetry algebra is then defined using the ansatz

$$
L_{n}^{Q}=-R_{\chi} e^{-\frac{i n \chi}{R_{\chi}}} \partial_{\chi}-i r e^{-\frac{i n \chi}{R_{\chi}}} \partial_{r}+\text { (subleading terms) }
$$

along the gauge Kaluza-Klein direction. The same reasoning leading to the $S L(2, \mathbb{Z})$ family of Virasoro generators (119) would then apply as well. The existence of such a Virasoro symmetry around the Kerr-Newman black holes is corroborated by near-extremal scattering amplitudes as we will discuss in Section 5, and by the hidden conformal symmetry of probes, as we will discuss in Section 6.

\subsection{Absence of $S L(2, \mathbb{R})$ asymptotic symmetries}

The boundary conditions discussed so far do not admit solutions with non-trivial charges under the $S L(2, \mathbb{R})$ exact symmetry group of the background geometry generated by $\zeta_{0, \pm 1}(29)$. In fact, the boundary conditions are not even invariant under the action of the generator $\zeta_{1}$. One could ask the question if such an enlargement of boundary conditions is possible, which would open the 
possibility of enlarging the asymptotic-symmetry group to include the $S L(2, \mathbb{R})$ group and even a Virasoro extension thereof. We will now argue that such enlargement would result in trivial charges, which would not belong to the asymptotic-symmetry group.

First, we saw in Section 2.7 that there is a class of near-extremal solutions (79) obeying the boundary conditions $(111)-(116)$ with near-horizon energy $\not \mathcal{Q}_{\partial_{t}}=T^{\text {near-ext }} \delta \mathcal{S}_{\text {ext }}$. However, the charge $\not \mathcal{Q}_{\partial_{t}}$ is a heat term, which is not integrable when both $T^{\text {near-ext }}$ and $\mathcal{S}_{\text {ext }}$ can be varied. Moreover, upon scaling the coordinates as $t \rightarrow t / \alpha$ and $r \rightarrow \alpha r$ using the $S L(2, \mathbb{R})$ generator (24), one obtains the same metric as (79) with $T^{\text {near-ext }} \rightarrow T^{\text {near-ext }} / \alpha$. If one would allow the class of near-extremal solutions $(79)$ and the presence of $S L(2, \mathbb{R})$ symmetries in a consistent set of boundary conditions, one would be forced to fix the entropy $\mathcal{S}_{\text {ext }}$ to a constant, in order to define integrable charges. The resulting vanishing charges would not belong to the asymptotic-symmetry algebra. Since there is no other obvious candidate for a solution with non-zero near-horizon energy, we argued in Section 2.9 that there is no such solution at all. If that assumption is correct, the $S L(2, \mathbb{R})$ algebra would always be associated with zero charges and would not belong to the asymptotic symmetry group. Hence, no additional non-vanishing Virasoro algebra could be derived in a consistent set of boundary conditions. For alternative points of view, see [215, 216, 236, 214].

Second, as far as extremal geometries are concerned, there is no need for a non-trivial $S L(2, \mathbb{R})$ or second Virasoro algebra. As we will see in Section 4.4, the entropy of extremal black holes will be matched using a single copy of the Virasoro algebra, using the assumption that Cardy's formula applies. Matching the entropy of non-extremal black holes and justifying Cardy's formula requires two Virasoro algebras, as we will discuss in Section 6.6. However, non-extremal black holes do not admit a near-horizon limit and, therefore, are not dynamical objects described by a consistent class of near-horizon boundary conditions. At most, one could construct the near horizon region of nonextremal black holes in perturbation theory as a large deformation of the extremal near-horizon geometry. This line of thought was explored in [67]. In the context of the near-extremal Kerr black hole, it was obtained using a dimensionally-reduced model such that the algebra of diffeomorphisms, which extends the $S L(2, \mathbb{R})$ algebra, is represented on the renormalized stress-energy tensor as a Virasoro algebra. It would be interesting to further define and extend these arguments (which go beyond a standard asymptotic-symmetry analysis) to non-dimensionally-reduced models and to other near-extremal black holes.

Finally, let us also note that the current discussion closely parallels the lower dimensional example of the near-horizon limit of the extremal BTZ black hole discussed in [30]. There it was shown that the near-horizon geometry of the extremal BTZ black hole of angular momentum $J$ is a geometry with $S L(2, \mathbb{R}) \times U(1)$ isometry

$$
d s^{2}=\frac{l^{2}}{4}\left(-r^{2} d t^{2}+\frac{d r^{2}}{r^{2}}+2 J\left(d \phi+\frac{r}{\sqrt{2 J}} d t\right)^{2}\right),
$$

with $\phi \sim \phi+2 \pi$, which is known as the self-dual $\mathrm{AdS}_{3}$ orbifold [100]. It was found in [30] that the asymptotic symmetry group consists of one chiral Virasoro algebra extending the $U(1)$ symmetry along $\partial_{\phi}$, while the charges associated with the $S L(2, \mathbb{R})$ symmetry group are identically zero. These observations are consistent with the analysis of four-dimensional near-horizon geometries (25), whose constant $\theta$ sections share similar qualitative features with the three-dimensional geometries (121). It was also shown that an extension of the boundary conditions exists that is preserved by a second Virasoro algebra extending the $S L(2, \mathbb{R})$ exact-symmetry algebra [24]. All associated charges can be argued to be zero, but a non-trivial central extension still appears as a background charge when a suitable regularization is introduced. However, the regularization procedure does not generalize to the four-dimensional geometries mainly because two features of the three-dimensional geometry $(121)$ are not true in general $\left(\partial_{t}\right.$ is null in (121) and $\partial_{t}-\epsilon \partial_{\phi}$ with $\epsilon \ll 1$ is a global timelike Killing vector).

Living Reviews in Relativity

http://www. livingreviews.org/lrr-2012-11 


\subsection{Virasoro algebra and central charge}

Let us now assume in the context of the general theory (1) that a consistent set of boundary conditions exists that admit the Virasoro algebra generated by (107)-(108) as asymptotic-symmetry algebra. Current results are consistent with that assumption but, as emphasized earlier, boundary conditions have been checked only partially $[156,5,21]$.

Let us define the Dirac bracket between two charges as

$$
\left\{\mathcal{Q}_{\left(\zeta_{m}, \Lambda_{m}\right)}, \mathcal{Q}_{\left(\zeta_{n}, \Lambda_{n}\right)}\right\} \equiv-\delta_{\left(\zeta_{m}, \Lambda_{m}\right)} \mathcal{Q}_{\left(\zeta_{n}, \Lambda_{n}\right)}
$$

Here, the operator $\delta_{\left(\zeta_{m}, \Lambda_{m}\right)}$ is a derivative in phase space that acts on the fields $g_{\mu \nu}, A_{\mu}^{I}, \chi^{A}$ appearing in the charge $\mathcal{Q}$ as (104). From general theorems in the theory of asymptotic-symmetry algebras [59, 35, 36], the Dirac bracket represents the asymptotic symmetry algebra up to a central term, which commutes with each element of the algebra. Namely, one has

$$
\left\{\mathcal{Q}_{\left(\zeta_{m}, \Lambda_{m}\right)}, \mathcal{Q}_{\left(\zeta_{n}, \Lambda_{n}\right)}\right\}=\mathcal{Q}_{\left[\left(\zeta_{m}, \Lambda_{m}\right),\left(\zeta_{n}, \Lambda_{n}\right)\right]}+\mathcal{K}_{\left(\zeta_{m}, \Lambda_{m}\right),\left(\zeta_{n}, \Lambda_{n}\right)},
$$

where the bracket between two generators has been defined in $(105)$ and $\mathcal{K}$ is the central term, which is anti-symmetric in its arguments. Furthermore, using the correspondence principle in semiclassical quantization, Dirac brackets between generators translate into commutators of quantum operators as $\{\ldots\} \rightarrow-\frac{i}{\hbar}[\ldots]$. Note that, according to this rule, the central terms in the algebra aquire a factor of $1 / \hbar$ when operator eigenvalues are expressed in units of $\hbar$ (or equivalently, when one performs $\mathcal{Q} \rightarrow \hbar \mathcal{Q}$ and divide both sides of (123) by $\hbar$.).

For the case of the Virasoro algebra (110), it is well known that possible central extensions are classified by two numbers $c$ and $A$. The general result has the form

$$
\left[\mathcal{L}_{m}, \mathcal{L}_{n}\right]=(m-n) \mathcal{L}_{m+n}+\frac{c}{12} m\left(m^{2}-A\right) \delta_{m,-n},
$$

where $A$ is a trivial central extension that can be set to 1 by shifting the background value of the charge $\mathcal{L}_{0}$. The non-trivial central extension $c$ is a number that is called the central charge of the Virasoro algebra. From the theorems [59, 35, 36], the central term in (123) can be expressed as a specific and known functional of the Lagrangian $\mathcal{L}$ (or equivalently of the Hamiltonian), the background solution $\bar{\phi}=\left(\bar{g}_{\mu \nu}, \bar{A}_{\mu}^{I}, \bar{\chi}^{A}\right)$ (the near-horizon geometry in this case) and the Virasoro generator $(\zeta, \Lambda)$ around the background

$$
c=c(\mathcal{L}, \bar{\phi},(\zeta, \Lambda)) .
$$

In particular, the central charge does not depend on the choice of boundary conditions. The representation theorem leading to (124) only requires that such boundary conditions exist. The representation theorem for asymptotic Hamiltonian charges [59] was famously first applied [58] to Einstein's gravity in three dimensions around AdS, where the two copies of the Virasoro asymptoticsymmetry algebra were shown to be centrally extended with central charge $c=\frac{3 l}{2 G_{N} \hbar}$, where $l$ is the AdS radius and $G_{N}$ Newton's constant.

For the general near-horizon solution (25) of the Lagrangian (1) and the Virasoro ansatz (107)(108), one can prove $[159,97]$ that the matter part of the Lagrangian (including the cosmological constant) does not contribute directly to the central charge, but only influences the value of the central charge through the functions $\Gamma(\theta), \alpha(\theta), \gamma(\theta)$ and $k$, which solve the equations of motion. The central charge (125) is then given as the $\mathrm{m}^{3}$ factor of the following expression defined in terms of the fundamental charge formula of Einstein gravity as [35]

$$
c_{J}=\left.12 i \lim _{r \rightarrow \infty} \mathcal{Q}_{L_{m}}^{\text {Einstein }}\left[\mathcal{L}_{L_{-m}} \bar{g} ; \bar{g}\right]\right|_{m^{3}},
$$


where $\mathcal{L}_{L_{-m}} \bar{g}$ is the Lie derivative of the metric along $L_{-m}$ and

$$
\begin{gathered}
\mathcal{Q}_{L_{m}}^{\text {Einstein }}[h ; g] \equiv \frac{1}{8 \pi G_{N}} \int_{S} d S_{\mu \nu}\left(\xi^{\nu} D^{\mu} h+\xi^{\mu} D_{\sigma} h^{\sigma \nu}+\xi_{\sigma} D^{\nu} h^{\sigma \mu}+\frac{1}{2} h D^{\nu} \xi^{\mu}\right. \\
\left.+\frac{1}{2} h^{\mu \sigma} D_{\sigma} \xi^{\nu}+\frac{1}{2} h^{\nu \sigma} D^{\mu} \xi_{\sigma}\right) .
\end{gathered}
$$

Here, $d S_{\mu \nu}=\frac{1}{2(d-2) !} \sqrt{-g} \epsilon_{\mu \nu \alpha_{1} \ldots \alpha_{d-2}} d x^{\alpha_{1}} \wedge \cdots \wedge d x^{\alpha_{d-2}}$ is the integration measure in $d$ dimensions and indices are raised with the metric $g^{\mu \nu}, h \equiv g^{\mu \nu} h_{\mu \nu}$ and $S$ is a surface at fixed time and radius $r$. Physically, $Q_{\xi}^{\text {Einstein }}[h ; g]$ is defined as the charge of the linearized metric $h_{\mu \nu}$ around the background $g_{\mu \nu}$ associated with the Killing vector $\xi$, obtained from Einstein's equations [1]. Substituting the general near-horizon solution (25) and the Virasoro ansatz (107) - (108), one obtains

$$
c_{J}=\frac{3 k}{G_{N} \hbar} \int_{0}^{\pi} d \theta \alpha(\theta) \Gamma(\theta) \gamma(\theta) .
$$

We will drop the factors of $G_{N}$ and $\hbar$ from now on. In the case of the NHEK geometry in Einstein gravity, substituting (37), one finds the simple result [156]

$$
c_{J}=12 J
$$

The central charge of the Virasoro ansatz (107) - (108) around the Kerr-Newman black hole turns out to be identical to (129). We note in passing that the central charge $c_{J}$ of extremal Kerr or Kerr-Newman is a multiple of six, since the angular momentum is quantized as a half-integer multiple of $\hbar$. The central charge can be obtained for the Kerr-Newman-AdS solution as well [159] and the result is

$$
c_{J}=\frac{12 a r_{+}}{\Delta_{0}}
$$

where $\Delta_{0}$ has been defined in (44).

When higher-derivative corrections are considered, the central charge can still be computed exactly, using as crucial ingredients the $S L(2, \mathbb{R}) \times U(1)$ symmetry and the $(t, \phi)$ reversal symmetry of the near-horizon solution. The result is given by [20]

$$
c_{J}=-12 k \int_{\Sigma} \frac{\delta^{\mathrm{cov}} L}{\delta R_{a b c d}} \epsilon_{a b} \epsilon_{c d} \operatorname{vol}(\Sigma)
$$

where the covariant variational derivative $\delta^{\mathrm{cov}} / \delta R_{a b c d}$ has been defined in (53) in Section 2.5. One caveat should be noted. The result [20] is obtained after auxiliary fields are introduced in order to rewrite the arbitrary diffeomorphism-invariant action in a form involving at most two derivatives of the fields. It was independently observed in [190] that the formalism of [35, 36] applied to the Gauss-Bonnet theory formulated using the metric only cannot reproduce the central charge (131) and, therefore, the black-hole entropy as will be developed in Section 4.4. One consequence of these two computations is that the formalism of $[35,36,90]$ is not invariant under field redefinitions. In view of the cohomological results of [35], this ambiguity can appear only in the asymptotic context and when certain asymptotic linearity constraints are not obeyed. Nevertheless, it has been acknowledged that boundary terms in the action should be taken into account [237, 164]. Adding supplementary terms to a well-defined variational principle amount to deforming the boundary conditions $[56,266,213]$ and modifying the symplectic structure of the theory through its coupling to the boundary dynamics [96]. Therefore, it remains to be checked if the prescription of [96] to include boundary effects would allow one to reconcile the work of [190] with that of [20].

In five-dimensional Einstein gravity coupled to $U(1)$ gauge fields and scalars, the central charge associated with the Virasoro generators along the direction $\partial_{\phi_{i}}, i=1,2$ can be obtained as a 
straightforward extension of $(128)[159,97]$. One has

$$
c_{\phi_{i}}=6 \pi k_{i} \int_{0}^{\pi} d \theta \alpha(\theta) \Gamma(\theta) \gamma(\theta),
$$

where the extra factor of $2 \pi$ with respect to (128) originates from integration around the extra circle (see also $[151,166]$ for some higher derivative corrections). Since the entropy (54) is invariant under a $S L(2, \mathbb{Z})$ change of basis of the torus coordinates $\left(\phi_{1}, \phi_{2}\right)$ as (118), $c_{\phi_{i}}$ transforms under a modular transformation as $k_{i}$. Now, $k_{i}$ transforms in the same fashion as the coordinate $\phi_{i}$, as can be deduced from the form of the near-horizon geometry (36). Then, the central charge for the Virasoro ansatz (119) is given by

$$
c_{\left(p_{1}, p_{2}\right)}=p_{1} c_{\phi_{1}}+p_{2} c_{\phi_{2}} .
$$

Let us now discuss the central extension of the alternative Virasoro ansatz (120) for the extremal Reissner-Nordström black hole of electric charge $Q$ and mass $Q$. First, the central charge is inversely proportional to the scale $R_{\chi}$ set by the Kaluza-Klein direction that geometrizes the gauge field. One can see this as follows. The central charge is bilinear in the Virasoro generator and, therefore, it gets a factor of $\left(R_{\chi}\right)^{2}$. Also, the central charge consists of the $n^{3}$ term of the formula (127), it then contains terms admitting three derivatives along $\chi$ of $e^{-i n \chi / R}$ and, therefore, it contains a factor of $R_{\chi}^{-3}$. Also, the central charge is defined as an integration along $\chi$ and, therefore, it should contain one factor $R_{\chi}$ from the integration measure. Finally, the charge is inversely proportional to the five-dimensional Newton's constant $G_{5}=\left(2 \pi R_{\chi}\right) G_{4}$. Multiplying this complete set of scalings, one obtains that the central charge is inversely proportional to the scale $R_{\chi}$.

Using the simple embedding of the metric and the gauge fields in a higher-dimensional spacetime (2), as discussed in Section 1.2, and using the Virasoro ansatz (120), it was shown [159, 146, 77] that the central charge formula (126) gives

$$
c_{Q}=\frac{6 Q^{3}}{R_{\chi}} .
$$

One might object that (2) is not a consistent higher-dimensional supergravity uplift. Indeed, as we discussed in Section 1.2, one should supplement matter fields such as (3). However, since matter fields such as scalars and gauge fields do not contribute to the central charge (125) [97], the result (134) holds for any such consistent embedding.

Similarly, we can uplift the Kerr-Newman black hole to five-dimensions, using the uplift (2)(3) and the four-dimensional fields (25) - (39). Computing the central charge (132) for the Virasoro ansatz (120), we find again ${ }^{13}$

$$
c_{Q}=\frac{6 Q^{3}}{R_{\chi}} .
$$

Under the assumption that the $U(1)$ gauge field can be uplifted to a Kaluza-Klein direction, we can also formulate the Virasoro algebra (119) and associated boundary conditions for any circle related by an $S L(2, \mathbb{Z})$ transformation of the torus $U(1)^{2}$. Applying the relation (133) we obtain the central charge

$$
c_{\left(p_{1}, p_{2}\right)}=p_{1} c_{J}+p_{2} c_{Q}=6\left(p_{1}(2 J)+p_{2} \frac{Q^{3}}{R_{\chi}}\right) .
$$

Let us discuss the generalization to AdS black holes. As discussed in Section 1.2, one cannot use the ansatz (2) to uplift the $U(1)$ gauge field. Rather, one can uplift to eleven dimensions along

${ }^{13}$ We thank Tom Hartman for helping deriving this central charge during a private communication. 
a seven-sphere. One can then argue, as in [204], that the only contribution to the central charge comes from the gravitational action. Even though no formal proof is available, it is expected that it will be the case given the results for scalar and gauge fields in four and five dimensions [97]. Applying the charge formula (126) accounting for the gravitational contribution of the complete higher-dimensional spacetime, one obtains the central charge for the Virasoro algebra (120) as [204]

$$
c_{Q}=6 Q_{e} \frac{r_{+}^{2}-a^{2}}{\Xi \Delta_{0} R_{\chi}},
$$

where parameters have been defined in Section 2.4.4 and $2 \pi R_{\chi}$ is the length of the $U(1)$ circle in the seven-sphere.

The values of the central charges (129), (130), (131), (132), (133), (135), (136), (137) are the main results of this section.

\subsection{Microscopic counting of the entropy}

In Section 4.3 we have shown the existence of an asymptotic Virasoro algebra at the boundary $r=\infty$ of the near-horizon geometry. We also discussed that the $S L(2, \mathbb{R})$ symmetry is associated with zero charges. Following semi-classical quantization rules, the operators that define quantum gravity with the boundary conditions (111), (116), (115) form a representation of the Virasoro algebra and are in a ground state with respect to the representation of the $S L(2, \mathbb{R})$ symmetry $[251,156]$. A consistent theory of quantum gravity in the near-horizon region, if it can be defined at all, is therefore either a chiral CFT or a chiral half of a two-dimensional CFT. A chiral CFT is defined as a holomorphically-factorized CFT with zero central charge in one sector, while a chiral half of a $2 d$ CFT can be obtained, e.g., after a chiral limit of a $2 d$ CFT, see Section 3.2. We will see in Sections 5 and 6 that the description of non-extremal black holes favors the interpretation of quantum gravity in extremal black holes as the chiral half of a full-fledged two-dimensional CFT. Moreover, the applicability of Cardy's formula as detailed later on also favors the existence of a two-dimensional CFT. Since the near-horizon geometry is obtained as a strict near-horizon limit of the original geometry, the CFT might be thought of as describing the degrees of freedom of the black-hole horizon.

Before moving further on, let us step back and first review an analogous reasoning in $\mathrm{AdS}_{3}$ [251]. In the case of asymptotically $\mathrm{AdS}_{3}$ spacetimes, the asymptotic symmetry algebra contains two Virasoro algebras. Also, one can define a two-dimensional flat cylinder at the boundary of $\mathrm{AdS}_{3}$ using the Fefferman-Graham theorem [137]. One is then led to identify quantum gravity in $\mathrm{AdS}_{3}$ spacetimes with a two-dimensional CFT defined on the cylinder. The known examples of AdS/CFT correspondences involving $\mathrm{AdS}_{3}$ factors can be understood as a correspondence between an ultraviolet completion of quantum gravity on $\mathrm{AdS}_{3}$ and a specific CFT. The vacuum $\mathrm{AdS}_{3}$ spacetime is more precisely identified with the $S L(2, \mathbb{R}) \times S L(2, \mathbb{R})$ invariant vacuum of the CFT, which is separated with a mass gap of $-c / 24$ from the zero-mass black holes. Extremal black holes with $\mathrm{AdS}_{3}$ asymptotics, the extremal BTZ black holes [28], are thermal states in the dual CFT with one chiral sector excited and the other sector set to zero temperature. It was further understood in [30] that taking the near-horizon limit of the extremal BTZ black hole corresponds to taking the DLCQ of the dual CFT (see Section 3.2 for a review of the DLCQ procedure and [31, 151] for further supportive studies). The resulting CFT is chiral and has a frozen $S L(2, \mathbb{R})$ right sector.

Given the close parallels between the near-horizon geometry of the extremal BTZ black hole (121) and the near-horizon geometries of four-dimensional extremal black holes (25), it has been suggested in [30] that extremal black holes are described by a chiral limit of two-dimensional CFT. This assumption nicely accounts for the fact that only one Virasoro algebra appears in the asymptotic symmetry algebra and it is consistent with the conjecture that no non-extremal excitations are allowed in the near-horizon limit as we discussed earlier. Moreover, the assumption that the

Living Reviews in Relativity

http://www. livingreviews.org/lrr-2012-11 
chiral half of the CFT originates from a limiting DLCQ procedure is consistent with the fact that there is no natural $S L(2, \mathbb{R}) \times S L(2, \mathbb{R})$ invariant geometry in the boundary conditions (111), which would be dual to the vacuum state of the CFT. Indeed, even in the three-dimensional example, the geometric dual to the vacuum state (the $\mathrm{AdS}_{3}$ geometry) does not belong to the phase space defined in the near-horizon limit of extremal black holes. It remains an enigma why there is no natural $S L(2, \mathbb{R}) \times S L(2, \mathbb{R})$ invariant geometry in gravity at all that is dual to the vacuum state.

Let us now take as an assumption that the near-horizon geometry of the extremal Kerr black

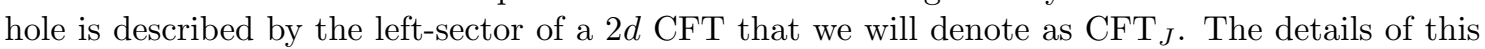
CFT will depend on the ultraviolet completion of gravity, but these details will be (fortunately) unimportant here. Instead, we will show that one can account for the entropy using the universal properties of that CFT. First, we can identify a non-trivial temperature for the excited states. We saw in Section 2.6 that scalar quantum fields in the analogue of the Frolov-Thorne vacuum restricted to extremal excitations have the temperature (67). Individual modes are co-rotating with the black hole along $\partial_{\phi}$. Since we identify the left-sector of the CFT with excitations along $\partial_{\phi}$ and the right $S L(2, \mathbb{R})_{R}$ sector is frozen, the CFT left-moving states are described by a thermal density matrix with temperatures

$$
T_{L}=T_{\phi}, \quad T_{R}=0,
$$

where $T_{\phi}$ is given in (67). The other quantities $T_{e}$ and $T_{m}$ defined in (61) are then better interpreted as being proportional to auxiliary chemical potentials. One can indeed rewrite the Boltzman factor $(66)$ as

$$
\exp \left(-\hbar \frac{m-q_{e} \mu_{L}^{J, e}-q_{m} \mu_{L}^{J, m}}{T_{L}}\right)
$$

where the left chemical potentials are defined as

$$
\mu_{L}^{J, e} \equiv-\frac{T_{\phi}}{T_{e}}, \quad \mu_{L}^{J, m} \equiv-\frac{T_{\phi}}{T_{m}} .
$$

It is remarkable that applying blindly Cardy's formula (90) using the central charge $c_{L}=c_{J}$ given in (129) and using the temperatures (138), one reproduces the extremal Bekenstein-Hawking black-hole entropy

$$
\mathcal{S}_{\mathrm{CFT}} \stackrel{!}{=} \mathcal{S}_{\mathrm{ext}},
$$

as first shown in [156]. This matching is clearly not a numerical coincidence. For any spinning extremal black hole of the theory (1), one can associate a left-moving Virasoro algebra of central charge $c_{L}=c_{J}$ given in (128). The black-hole entropy (50) is then similarly reproduced by Cardy's formula (141). As remarkably, taking any higher curvature correction to the gravitational Lagrangian into account, one also reproduces the Iyer-Wald entropy (52) using Cardy's formula, while the central charge (131) is computed (apparently) completely independently from the entropy!

One can easily be puzzled by the incredible matching (141) valid for virtually any extremal black hole and outside the usual Cardy regime, as discussed in Section 3.1. Indeed, there are no arguments for unitarity and modular invariance of the dual CFT. It might suggest that Cardy's formula has a larger range of applicability than what has been proven so far. Alternatively, this might suggest the existence of a long string CFT, as reviewed in Section 3.3. Note also that the central charge depends on the black-hole parameters, such as the angular momentum or the electric charge. This is not too surprising since, in known AdS/CFT correspondences where the black hole contains an $\mathrm{AdS}_{3}$ factor in the near-horizon geometry, the Brown-Henneaux central charge $c=3 l / 2 G_{3}[58]$ also depends on the parameters of the black hole because the AdS length $l$ is a function of the black hole's charge [206]. 
Let us now add an additional dimension to the scope of microscopic models. It turns out that when electromagnetic fields are present, another CFT description is available. Instead of assigning the left-moving temperature as (138), one might instead emphasize that electricallycharged particles are immersed in a thermal bath with temperature $T_{\chi}=R_{\chi} T_{e}$, as derived in (70) in Section 2.6. Identifying the left sector of the dual field theory with a density matrix at temperature $T_{\chi}$ and assuming again no right excitations at extremality, we make the following assignment

$$
T_{L}=T_{\chi}=R_{\chi} T_{e}, \quad T_{R}=0 .
$$

The other quantities $T_{\phi}$ and $T_{m}$ defined in (61) are then better interpreted as being proportional to auxiliary chemical potentials. One can indeed rewrite the Boltzman factor (66) as

$$
\exp \left(-\hbar \frac{q_{\chi}-m \mu_{L}^{Q, \phi}-q_{m} \mu_{L}^{Q, m}}{T_{\chi}}\right)
$$

where $q_{\chi}=R_{\chi} q_{e}$ is the probe electric charge in units of the Kaluza-Klein length and the left chemical potentials are defined as

$$
\mu_{L}^{Q, \phi} \equiv-\frac{T_{\chi}}{T_{\phi}}, \quad \mu_{L}^{Q, m} \equiv-\frac{T_{\chi}}{T_{m}}
$$

We argued above that in the near-horizon region, excitations along the gauge-field direction fall into representations of the Virasoro algebra defined in (120). As supported by non-extremal extensions of the correspondence discussed in Sections 5 and 6, the left sector of the dual field theory can be argued to be the chiral half of a $2 d$ CFT. Remarkably, Cardy's formula (90) with temperatures (142) and central charge (134) also reproduces the entropy of the Kerr-Newman black hole. When the angular momentum is identically zero, the black-hole entropy of the Reissner-Nordström black hole $\mathcal{S}_{\text {ext }}=\pi Q^{2}$ is then reproduced from Cardy's formula with left central charge $c_{L}=c_{Q}$ given in (134) and left temperature $T_{L}=R_{\chi} /(2 \pi Q)$ as originally obtained in [159]. As one can easily check, the entropy of the general Kerr-Newman-AdS black hole can be similarily reproduced, as shown in $[79,71,78,76,82]$. We will refer to the class of CFTs with Virasoro algebra (120) by the acronym $\mathrm{CFT}_{Q}$. Note that the entropy matching does not depend on the scale of the Kaluza-Klein dimension $R_{\chi}$, which is arbitrary in our analysis.

Finally, when two $U(1)$ symmetries are present, one can apply a modular transformation mixing the two $U(1)$ and one obtains a different CFT description for each choice of $S L(2, \mathbb{Z})$ element. Indeed, we argued that the set of generators (119) obeys the Virasoro algebra with central charge (136). After performing an $S L(2, \mathbb{Z})$ change of basis in the Boltzman factor (66), we deduce the temperature of the CFT and Cardy's formula is similarly reproduced. We will denote the corresponding class of CFTs by the acronym $\mathrm{CFT}_{\left(p_{1}, p_{2}, p_{3}\right)}$. 


\section{$5 \quad$ Scattering from Near-Extremal Black Holes}

In Section 4, we presented how the entropy of any extremal black hole can be reproduced microscopically from one chiral half of one (or several) two-dimensional CFT(s). In this section, we will present arguments supporting the conjecture that this duality can be extended to near-extremal black holes dual to a CFT with a second sector slightly excited, following $[53,106,160]$. We will show that the derivation of $[53,106,160]$ is supporting evidence for all CFTs presented in Section 4 , as noted in $[79,71]$. In the case of the $\mathrm{CFT}_{J}$ dual to near-extremal spinning black holes, one can think intuitively that the second CFT sector is excited for the following reason: lights cones do not quite coalesce at the horizon, so microscopic degrees of freedom do not rotate at the speed of light along the single axial direction. The intuition for the other CFTs $\left(\mathrm{CFT}_{Q}, \mathrm{CFT}_{\left(p_{1}, p_{2}, p_{3}\right)}\right)$ is less immediate.

Near-extremal black holes are defined as black holes with a Hawking temperature that is very small compared with their inverse mass

$$
M T_{H} \ll 1 .
$$

At finite energy away from extremality, one cannot isolate a decoupled near-extremal near-horizon geometry. As we discussed in Section 4, the extremal near-horizon geometry then suffers from infrared divergences, which destabilize the near-horizon geometry. This prevents one to formulate boundary conditions à la Brown-Henneaux to describe non-chiral excitations. Therefore, another approach is needed.

If near-extremal black holes are described by a dual field theory, it means that all properties of these black holes - classical or quantum - can be derived from a computation in the dual theory, after it has been properly coupled to the surrounding spacetime. We now turn our attention to the study of one of the simplest dynamical processes around black holes: the scattering of a probe field. This route was originally followed for static extremal black holes in [208, 209]. In this approach, no explicit metric boundary conditions are needed. Moreover, since gravitational backreaction is a higher-order effect, it can be neglected. One simply computes the black-hole-scattering amplitudes on the black-hole background. In order to test the near-extremal black hole/CFT correspondence, one then has to determine whether or not the black hole reacts like a two-dimensional CFT to external perturbations originating from the asymptotic region far from the black hole.

We will only consider fields that probe the near-horizon region of near-extremal black holes. These probe fields have energy $\omega$ and angular momentum $m$ close to the superradiant bound $\omega \sim m \Omega_{J}^{\mathrm{ext}}+q_{e} \Phi_{e}^{\mathrm{ext}}$,

$$
M\left(\omega-m \Omega_{J}^{\mathrm{ext}}-q_{e} \Phi_{e}^{\mathrm{ext}}\right) \ll 1 .
$$

In order to simplify the notation, in this section we will drop all hats on quantities defined in the asymptotic region far from the black hole.

Since no general scattering theory around near-extremal black-hole solutions of (1) has been proposed so far, we will concentrate our discussion on near-extremal asymptotically-flat KerrNewman black holes, as discussed in [53, 160] (see also [79, 72, 81, 74, 77, 3]). Extensions to the Kerr-Newman-AdS black hole or other specific black holes in four and higher dimensions in gauged or ungauged supergravity can be found in [53, 106, 73, 242, 46] (see also [71, 80, 129, 224]).

\subsection{Near-extremal Kerr-Newman black holes}

Near-extremal Kerr-Newman black holes are characterized by their mass $M$, angular momentum $J=M a$ and electric charge $Q$. (We take $a, Q \geq 0$ without loss of generality.) They contain near-extremal Kerr and Reissner-Nordström black holes as particular instances. The metric and thermodynamic quantities can be found in many references and will not be reproduced here. 
The near-extremality condition (145) is equivalent to the condition that the reduced Hawking temperature is small,

$$
\tau_{H} \equiv \frac{r_{+}-r_{-}}{r_{+}} \ll 1
$$

Indeed, one has $\tau_{H}=M T_{H}\left[4 \pi\left(\left(r_{+} / M\right)^{2}+(a / M)^{2}\right) /\left(r_{+} / M\right)\right]$ and the term in between the brackets is of order one since $0 \leq a / M \leq 1,0 \leq Q / M \leq 1$ and $1 \leq r_{+} / M \leq 2$. Therefore, we can use interchangeably the conditions (145) and (147).

Since there is both angular momentum and electric charge, extremality can be reached both in the regime of vanishing angular momentum $J$ and vanishing electric charge $Q$. When angular momentum is present, we expect that the dynamics could be described by the $\mathrm{CFT}_{J}$, while when electric charge is present the dynamics could be described by the $\mathrm{CFT}_{Q}$. It is interesting to remark that the condition

$$
\frac{T_{H}}{\Omega_{J}} \ll 1
$$

implies $(145)-(147)$ since $\tau_{H}=\frac{T_{H}}{\Omega_{J}}(4 \pi a / M) \ll 1$ but it also implies $a>0$. Similarly, the condition

$$
M \frac{T_{H}}{\Phi_{e}} \ll 1
$$

implies $(145)-(147)$, since $\tau_{H}=4 \pi Q T_{H} / \Phi_{e}$, but it also implies $Q>0$. In the following, we will need only the near-extremality condition (145), and not the more stringent conditions (148) or (149). This is the first clue that the near-extremal scattering will be describable by both the $\mathrm{CFT}_{J}$ and the $\mathrm{CFT}_{Q}$.

Near-extremal black holes are characterized by an approximative near-horizon geometry, which controls the behavior of probe fields in the window (146). Upon taking $T_{H}=O(\lambda)$ and taking the limit $\lambda \rightarrow 0$ the near-horizon geometry decouples, as we saw in Section 2.7.

Probes will penetrate the near-horizon region close to the superradiant bound (146). When $T_{H}=O(\lambda)$ we need

$$
\omega=m \Omega_{J}^{\mathrm{ext}}+q_{e} \Phi_{e}^{\mathrm{ext}}+O(\lambda)
$$

Indeed, repeating the reasoning of Section 2.6, we find that the Boltzman factor defined in the near-horizon vacuum (defined using the horizon generator) takes the following form

$$
e^{\hbar \frac{\omega-m \Omega_{J}-q_{e} \Phi_{e}}{T_{H}}}=e^{-\hbar n} e^{-\hbar \frac{m}{T_{\phi}}-\hbar \frac{q_{e}}{T_{e}}},
$$

where $\omega, m$ and $q_{e}$ are the quantum numbers defined in the exterior asymptotic region and

$$
n \equiv \frac{\omega-m \Omega_{J}^{\mathrm{ext}}-q_{e} \Phi_{e}^{\mathrm{ext}}}{T_{H}}
$$

is finite upon choosing (150). The conclusion of this section is that the geometries (79) control the behavior of probes in the near-extremal regime (145)-(146). We identified the quantity $n$ as a natural coefficient defined near extremality. It will have a role to play in later Sections 5.3 and 5.4. We will now turn our attention to how to solve the equations of motion of probes close to extremality.

\subsection{Macroscopic greybody factors}

The problem of scattering of a general spin field from a Kerr black hole was solved in a series of classic papers by Starobinsky [248], Starobinsky and Churilov [249] and Press and Teukolsky [255, $256,235,257]$ in the early 1970 s (see also $[145,4,122]$ ). The scattering of a spin 0 and $1 / 2$ field 
from a Kerr-Newman black hole has also been solved [257], while the scattering of spins 1 and 2 from the Kerr-Newman black hole has not been solved to date.

Let us review how to solve this classic scattering problem. First, one has to realize that the Kerr-Newman black hole enjoys a remarkable property: it admits a Killing-Yano tensor [269, 232, 142]. (For a review and some surprising connections between Killing-Yano tensors and fermionic symmetries, see [148].) A Killing-Yano tensor is an anti-symmetric tensor $f_{\mu \nu}=-f_{\nu \mu}$, which obeys

$$
\nabla_{(\lambda} f_{\mu) \nu}=0 .
$$

This tensor can be used to construct a symmetric Killing tensor

$$
K_{\mu \nu}=f_{\mu}^{\lambda} f_{\lambda \nu}, \quad \nabla_{(\lambda} K_{\mu \nu)}=0,
$$

which is a natural generalization of the concept of Killing vector $K_{\mu}$ (obeying $\nabla_{(\mu} K_{\nu)}=0$ ). This Killing tensor was first used by Carter in order to define an additional conserved charge for geodesics [65]

$$
Q=K_{\mu \nu} \dot{x}^{\mu} \dot{x}^{\nu},
$$

and thereby reduce the geodesic equations in Kerr to first-order equations. More importantly for our purposes, the Killing tensor allows one to construct a second-order differential operator $K^{\mu \nu} \nabla_{\mu} \nabla_{\nu}$, which commutes with the Laplacian $\nabla^{2}$. This allows one to separate the solutions of the scalar wave equation $\nabla^{2} \Psi^{s=0}=0$ as [65]

$$
\Psi^{s=0}=e^{-i \omega t+i m \phi} S_{\omega, A, m}(\theta) R_{\omega, A, m}(r),
$$

where $A$ is the real separation constant present in both equations for $S(\theta)$ and $R(r)$. The underlying Killing-Yano tensor structure also leads to the separability of the Dirac equation for a probe fermionic field. For simplicity, we will not discuss further fermionic fields here and we refer the interested reader to the original reference [160] (see also [41]). The equations for spin 1 and 2 probes in Kerr can also be shown to be separable after one has conveniently reduced the dynamics to a master equation for a master scalar $\Psi^{s}$, which governs the entire probe dynamics. As a result, one has

$$
\Psi^{s}=e^{-i \omega t+i m \phi} S_{\omega, A, m}^{s}(\theta) R_{\omega, A, m}^{s}(r) .
$$

The master scalar is constructed from the field strength and from the Weyl tensor for spin 1 $(s= \pm 1)$ and spin $2(s= \pm 2)$ fields, respectively, using the Newman-Penrose formalism. For the Kerr-Newman black hole, all attempts to separate the equations for spin 1 and spin 2 probes have failed. Hence, there is no known analytic method to solve those equations (for details, see [70]). Going back to Kerr, given a solution to the master scalar field equation, one can then in principle reconstruct the gauge field and the metric from the Teukolsky functions. This non-trivial problem was778ikm solved right after Teukolsky's work [89, 87]; see Appendix C of [122] for a modern review (with further details and original typos corrected).

In summary, for all separable cases, the dynamics of probes in the Kerr-Newman geometry can be reduced to a second-order equation for the angular part of the master scalar $S_{\omega, A, m}^{s}(\theta)$ and a second-order equation for the radial part of the master scalar $R_{\omega, A, m}^{s}(r)$. Let us now discuss their solutions after imposing regularity as boundary conditions, which include ingoing boundary conditions at the horizon. We will limit our discussion to the non-negative integer spins $s=0,1,2$ in what follows.

The angular functions $S_{\omega, A, m}^{s}(\theta)$ obey the spin-weighted spheroidal harmonic equation

$$
\begin{gathered}
{\left[\frac{1}{\sin \theta} \partial_{\theta}\left(\sin \theta \partial_{\theta}\right)-a^{2}\left(\omega^{2}-\mu^{2} \delta_{s, 0}\right) \sin ^{2} \theta-2 a \omega s \cos \theta-\frac{(m+s \cos \theta)^{2}}{\sin ^{2} \theta}\right.} \\
+A] S_{\omega, A, m}^{s}(\theta)=0 .
\end{gathered}
$$


(The Kronecker $\delta_{s, 0}$ is introduced so that the multiplicative term only appears for a massive scalar field of mass $\mu$.) All harmonics that are regular at the poles can be obtained numerically and can be classified by the usual integer number $l$ with $l \geq|m|$ and $l \geq|s|$. In general, the separation constant $A=A_{a \omega, l, m}^{s}$ depends on the product $a \omega$, on the integer $l$, on the angular momentum of the probe $m$ and on the spin $s$. At zero energy $(\omega=0)$, the equation reduces to the standard spinweighted spherical-harmonic equation and one simply has $A_{0, l, m}^{s}=l(l+1)-s^{2}$. For a summary of analytic and numerical results, see [44].

Let us now take the values $A_{a \omega, l, m}^{s}$ as granted and turn to the radial equation. The radial equation reduces to the following Sturm-Liouville equation

$$
\left[\Delta^{-s} \partial_{r}\left(\Delta^{s+1} \partial_{r}\right)-V^{s}(r)\right] R^{s}(r)=0
$$

where $\Delta(r)=\left(r-r_{+}\right)\left(r-r_{-}\right)=r^{2}-2 M r+a^{2}+Q^{2}$ in a potential $V^{s}(r)$. The form of the potential is pretty intricate. For a scalar field of mass $\mu$, the potential $V^{0}(r)$ is real and is given by

$$
V^{0}(r)=-\frac{H^{2}(r)}{\Delta(r)}-2 a m \omega+A_{a \omega, l, m}^{0}+\mu^{2}\left(r^{2}+a^{2}\right),
$$

where $H(r)=\omega\left(r^{2}+a^{2}\right)-q_{e} Q r-a m$. For a field of general spin on the Kerr geometry, the potential is, in general, complex and reads as

$$
V^{s}(r)=-\frac{H^{2}(r)-2 i s(r-M) H(r)}{\Delta(r)}-4 i s \omega r-2 a m \omega+A_{a \omega, l, m}^{s}-s(s+1),
$$

where $H(r)=\omega\left(r^{2}+a^{2}\right)-a m$. This radial equation obeys the following physical boundary condition: we require that the radial wave has an ingoing group velocity - or, in other words, is purely ingoing - at the horizon. This is simply the physical requirement that the horizon cannot emit classical waves. This also follows from a regularity requirement. The solution is then unique up to an overall normalization. For generic parameters, the Sturm-Liouville equation (159) cannot be solved analytically and one has to use numerical methods.

For each frequency $\omega$ and spheroidal harmonic $(l, m)$, the scalar field can be extended at infinity into an incoming wave and an outgoing wave. The absorption probability $\sigma_{\text {abs }}$ or macroscopic greybody factor is then defined as the ratio between the absorbed flux of energy at the horizon and the incoming flux of energy from infinity,

$$
\sigma_{\mathrm{abs}}(\omega, l, m, s ; M, a, Q)=\frac{d E_{\mathrm{abs}} / d t}{d E_{\mathrm{in}} / d t} .
$$

An important feature is that in the superradiant range (13) the absorption probability turns out to be negative, which results in stimulated as well as spontaneous emission of energy, as we reviewed in Section 2.1.

\subsection{Macroscopic greybody factors close to extremality}

The Sturm-Liouville problem (159) cannot be solved analytically. However, in the regime of nearextremal excitations (145)-(146) an approximative solution can be obtained analytically using asymptotic matched expansions: the wave equation is solved in the near-horizon region and in the far asymptotically-flat region and then matched along their common overlap region.

For that purpose, it is useful to define the dimensionless horizon radius $x=\left(r-r_{+}\right) / r_{+}$such that the outer horizon is at $x=0$. The two other singular points of the radial equation (159) are the inner horizon $x=-\tau_{H}$ and spatial infinity $x=\infty$. One then simply partitions the radial axis into two regions with a large overlap as 
- Near-horizon region: $x \ll 1$,

- Far region: $x \gg \tau_{H}$,

- Overlap region: $\tau_{H} \ll x \ll 1$.

The overlap region is guaranteed to exist thanks to (147).

In the near-extremal regime, the absorption probability $\sigma_{\text {abs }}$ gets a contribution from each region as

$$
\begin{aligned}
\sigma_{\mathrm{abs}} & =\sigma_{\mathrm{abs}}^{\mathrm{near}} \sigma_{\mathrm{abs}}^{\mathrm{match}}, \\
\sigma_{\mathrm{abs}}^{\mathrm{near}} & =\frac{d E_{\mathrm{abs}} / d t}{\left|\Psi\left(x=x_{B}\right)\right|^{2}}, \\
\sigma_{\mathrm{abs}}^{\text {match }} & =\frac{\left|\Psi\left(x=x_{B}\right)\right|^{2}}{d E_{\mathrm{in}} / d t},
\end{aligned}
$$

where $\left|\Psi\left(x=x_{B}\right)\right|^{2}$ is the norm of the scalar field in the overlap region with $\tau_{H} \ll x_{B} \ll 1$. One can conveniently normalize the scalar field such that it has unit incoming flux $d E_{\text {in }} / d t=1$. The contribution $\sigma_{\text {abs }}^{\text {match }}$ is then simply a normalization that depends on the coupling of the near-horizon region to the far region.

In the near-horizon region, the radial equation reduces to a much simpler hypergeometric equation. One can in fact directly obtain the same equation from solving for a probe in a nearextremal near-horizon geometry of the type (79), which is, as detailed in Section 2.3, a warped and twisted product of $\mathrm{AdS}_{2} \times S^{2}$. The presence of poles in the hypergeometric equation at $x=0$ and $x=-\tau_{H}$ requires one to choose the $\mathrm{AdS}_{2}$ base of the near-horizon geometry to be

$$
d s_{(2)}^{2}=-x\left(x+\tau_{H}\right) d t^{2}+\frac{d x^{2}}{x\left(x+\tau_{H}\right)} .
$$

One can consider the non-diagonal term $2 \Gamma(\theta) \gamma(\theta) k r d t d \phi$ appearing in the geometry (79) as a $U(1)$ electric field twisted along the fiber spanned by $d \phi$ over the $\mathrm{AdS}_{2}$ base space. It may then not be surprising that the dynamics of a probe scalar on that geometry can be expressed equivalently as a charged massive scalar on $\mathrm{AdS}_{2}$ with two electric fields: one coming from the $U(1)$ twist in the four-dimensional geometry, and one coming from the original $U(1)$ gauge field. By $S L(2, \mathbb{R})$ invariance, these two gauge fields are given by

$$
A_{1}=\alpha_{1} x d t, \quad A_{2}=\alpha_{2} x d t .
$$

The coupling between the gauge fields and the charged scalar is dictated by the covariant derivative

$$
\mathcal{D}=\nabla-i q_{1} A_{1}-i q_{2} A_{2}=\nabla-i q_{\mathrm{eff}} A,
$$

where $\nabla$ is the Levi-Civita connection on $\mathrm{AdS}_{2}$ and $q_{1}$ and $q_{2}$ are the electric charge couplings. One can rewrite more simply the connection as $q_{\mathrm{eff}} A$, where $q_{\mathrm{eff}}=q_{1} \alpha_{1}+q_{2} \alpha_{2}$ is the effective total charge coupling and $A=x d t$ is a canonically-normalized effective gauge field. The equation for a charged scalar field $\Phi(t, x)$ with mass $\mu_{\text {eff }}$ is then

$$
\mathcal{D}^{2} \Phi-\mu_{\text {eff }}^{2} \Phi=0 .
$$

Taking $\Phi(t, r)=e^{-i \omega_{\text {eff }} \tau_{H} t} \Phi(x)$, we then obtain the following equation for $\Phi(x)$,

$$
\left[\partial_{x}\left(x\left(x+\tau_{H}\right) \partial_{x}\right)+\frac{\left(\omega_{\mathrm{eff}} \tau_{H}+q_{\mathrm{eff}} x\right)^{2}}{x\left(x+\tau_{H}\right)}-\mu_{\mathrm{eff}}^{2}\right] \Phi(x)=0 .
$$


Using the field redefinition

$$
\Phi(x)=x^{s / 2}\left(\frac{x}{\tau_{H}}+1\right)^{s / 2} R^{s}(x),
$$

we obtain the equivalent equation,

$$
x\left(x+\tau_{H}\right) \partial_{x}^{2} R^{s}+(1+s)\left(2 x+\tau_{H}\right) \partial_{x} R^{s}+V(x) R^{s}=0,
$$

where the potential is

$$
V(x)=\frac{\left(a x+b \tau_{H}\right)^{2}-i s\left(2 x+\tau_{H}\right)\left(a x+b \tau_{H}\right)}{x\left(x+\tau_{H}\right)}-c .
$$

Here, the parameters $a, b, c$ are related to $\mu_{\mathrm{eff}}, q_{\mathrm{eff}}$ and $\omega_{\mathrm{eff}} \operatorname{as}^{14}$

$$
a=q_{\mathrm{eff}}+i s, \quad b=\omega_{\mathrm{eff}}+\frac{i s}{2}, \quad c=\mu_{\mathrm{eff}}^{2}-s .
$$

Finally, comparing Eq. (171) with (159), where the potential $V^{s}(r)$ is approximated by the nearhorizon potential, we obtain that these equations are identical, as previously announced, after identifying the parameters as

$$
\begin{aligned}
\omega_{\mathrm{eff}} & =\frac{n}{4 \pi}-\frac{i s}{2}, \\
q_{\mathrm{eff}} & =2 r_{+} \omega-q_{e} Q-i s, \\
\mu_{\mathrm{eff}}^{2} & =A_{a \omega, l, m}^{s}-2 a m \omega-s^{2}+\mu^{2}\left(r_{+}^{2}+a^{2}\right)-2 i m s .
\end{aligned}
$$

Moreover, using the expression of the frequency (150) near extremality, one can write the effective charge in the convenient form

$$
q_{\mathrm{eff}}=\frac{m}{2 \pi T_{\phi}}+\frac{q_{e}}{2 \pi T_{e}}-i s
$$

where the extremal Frolov-Thorne temperatures $T_{e}$ and $T_{\phi}$ are defined in (74).

We can now understand that there are two qualitatively distinct solutions for the radial field $R^{s}(x)$. Uncharged fields in $\mathrm{AdS}_{2}$ below a critical mass are unstable or tachyonic, as shown by Breitenlohner and Freedman [55]. Charged particles in an electric field on $\mathrm{AdS}_{2}$ have a modified Breitenlohner-Freedman bound

$$
m_{\mathrm{BF}}^{2}=-\frac{1}{4}+q_{\mathrm{eff}}^{2}
$$

in which the square mass is lifted up by the square charge. Below the critical mass, charged scalars will be unstable to Schwinger pair production [233, 184]. Let us define

$$
\beta^{2} \equiv \mu_{\mathrm{eff}}^{2}-m_{\mathrm{BF}}^{2}
$$

Stable modes will be characterized by a real $\beta \geq 0$, while unstable modes will be characterized by an imaginary $\beta$. This distinction between modes is distinct from superradiant and non-superradiant modes. Indeed, from the definition of $n$ (152), superradiance happens at near-extremality when $n<0$.

We can now solve the equation, impose the boundary conditions, compute the flux at the horizon and finally obtain the near-horizon absorption probability. The computation can be found in $[53,106,160]$. The net result is as follows. A massive, charge $e$, spin $s=0, \frac{1}{2}$ field with energy

\footnotetext{
14 There is a $\mathbb{Z}_{2}$ ambiguity in the definition of parameters since Eq. (171) is invariant upon replacing $(a, b, c)$ by $(i s+2 b-a, b, c+(2 b-i s)(i s+2 b-2 a))$. We simply chose one of the two identifications.
} 
$\omega$ and angular momentum $m$ and real $\beta>0$ scattered against a Kerr-Newman black hole with mass $M$ and charge $Q$ has near-region absorption probability

$$
\begin{aligned}
\sigma_{\mathrm{abs}}^{\text {near }} \sim & \frac{\left(T_{H}\right)^{2 \beta}\left(e^{\frac{n}{2}}-(-1)^{2 s} e^{-\frac{n}{2}}\right)}{\Gamma(2 \beta)^{2}}\left|\Gamma\left(\frac{1}{2}+\beta-s+i \operatorname{Re}\left(q_{\mathrm{eff}}\right)\right)\right|^{2} \\
& \left|\Gamma\left(\frac{1}{2}+\beta+i\left(\frac{n}{2 \pi}-\operatorname{Re}\left(q_{\mathrm{eff}}\right)\right)\right)\right|^{2} .
\end{aligned}
$$

For a massless spin $s=1,2$ field scattered against a Kerr black hole, exactly the same formula applies, but with $e=Q=0$. The absorption probability in the case where $\beta$ is imaginary can be found in the original papers [235, 257].

We will now show that the formulae (178) are Fourier transforms of CFT correlation functions. We will not consider the scattering of unstable fields with $\beta$ imaginary in this review. We refer the reader to [53] for arguments on how the scattering absorption probability of unstable spin 0 modes around the Kerr black hole match with dual CFT expectations.

\subsection{Microscopic greybody factors}

In this section we model the emission amplitudes from a microscopic point of view. We will first discuss near-extremal spinning black holes and we will extend our discussion to general charged and/or spinning black holes at the end of this section.

The working assumption of the microscopic model is that the near-horizon region of any nearextremal spinning black hole can be described and therefore effectively replaced by a dual twodimensional CFT. In the dual CFT picture, the near-horizon region is removed from the spacetime and replaced by a CFT glued along the boundary. Therefore, it is the near-horizon region contribution alone that we expect to be reproduced by the CFT. The normalization $\sigma_{\text {abs }}^{\text {match }}$ defined in (164) will then be dictated by the explicit coupling between the CFT and the asymptotically-flat region.

Remember from the asymptotic symmetry group analysis in Section 4.1 and 4.3 that boundary conditions were found where the exact symmetry of the near-horizon extremal geometry can be extended to a Virasoro algebra as

$$
U(1)_{L} \times S L(2, \mathbb{R})_{R} \rightarrow \operatorname{Vir}_{L} \times S L(2, \mathbb{R})_{R} .
$$

The right sector was taken to be frozen at extremality. The resulting chiral limit of the CFT with central charge $c_{J}=12 J$ sufficed to account for the extremal black-hole entropy.

We will now assume that quantum gravity states form a representation of both a left and a right-moving Virasoro algebra with generators $L_{n}$ and $\bar{L}_{n}$. The value of the right-moving central charge will be irrelevant for our present considerations. At near-extremality, the left sector is thermally excited at the extremal left-moving temperature (67). We take as an assumption that the right-moving temperature is on the order of the infinitesimal reduced Hawking temperature. As discussed in Sections 2.9 and 4.2, the presence of right-movers destabilize the near-horizon geometry. For the Kerr-Newman black hole, we have

$$
T_{L}=\frac{M^{2}+a^{2}}{4 \pi J}, \quad T_{R} \sim \tau_{H} .
$$

In order to match the bulk scattering amplitude for near-extremal Kerr-Newman black holes, the presence of an additional left-moving current algebra is required $[106,160]$. This current algebra is expected from the thermodynamic analysis of charged rotating extremal black holes. We indeed obtained in Section 2.6 and in Section 4.4 that such black holes are characterized by the chemical 
potential $\mu_{L}^{J, e}$ defined in (140) associated with the $U(1)_{e}$ electric current. Using the expressions (74), we find for the Kerr-Newman black hole the value

$$
\mu_{L}^{e}=-\frac{Q^{3}}{2 J}
$$

As done in [53], we also assume the presence of a right-moving $U(1)$ current algebra, whose zero eigenmode $\bar{J}_{0}$ is constrained by the level matching condition

$$
\bar{J}_{0}=L_{0} .
$$

The level matching condition is consistent with the fact that the excitations are labeled by three $\left(\omega, m, q_{e}\right)$ instead of four conserved quantities. The CFT state is then assumed to be at a fixed chemical potential $\mu_{R}$. This right-moving current algebra cannot be detected in the extremal nearhorizon geometry in the same way that the right-moving Virasoro algebra cannot be detected, so its existence is conjectural (see, however, [67]). This right-moving current algebra and the matching condition (182) will turn out to be adequate to match the gravitational result, as detailed below. Note that three-dimensional analogues of this level matching condition appeared in logically independent analyses [95, 94].

Therefore, under these assumptions, the symmetry group of the CFT dual to the near-extremal Kerr-Newman black hole is given by the product of a $U(1)$ current and a Virasoro algebra in both sectors,

$$
\left(\operatorname{Vir}_{L} \times \operatorname{Curr}_{L}\right) \times\left(\operatorname{Vir}_{R} \times \operatorname{Curr}_{R}\right) .
$$

In the description where the near-horizon region of the black hole is replaced by a CFT, the emission of quanta is due to couplings

$$
\Phi_{\text {bulk }} \mathcal{O}
$$

between bulk modes $\Phi_{\text {bulk }}$ and operators $\mathcal{O}$ in the CFT. The structure of the scattering cross section depends on the conformal weights $\left(h_{L}, h_{R}\right)$ and charges $\left(q_{L}, q_{R}\right)$ of the operator. The normalization of the coupling is also important for the normalization of the cross section.

The conformal weight $h_{R}$ can be deduced from the transformation of the probe field under the scaling $\bar{L}_{0}=t \partial_{t}-r \partial_{r}(24)$ in the overlap region $\tau_{H} \ll x \ll 1$. The scalar field in the overlap region is $\Phi \sim \Phi_{0}(t, \theta, \phi) r^{-\frac{1}{2}+\beta}+\Phi_{1}(t, \theta, \phi) r^{-\frac{1}{2}-\beta}$. Using the rules of the AdS/CFT dictionary [265], this behavior is related to the conformal weight as $\Phi \sim r^{h_{R}-1}, r^{-h_{R}}$. One then infers that [160]

$$
h_{R}=\frac{1}{2}+\beta \text {. }
$$

The values of the charges $\left(q_{L}, q_{R}\right)$ are simply the $U(1)$ charges of the probe,

$$
q_{L}=q_{e}, \quad q_{R}=m,
$$

where the charge $q_{R}=m$ follows from the matching condition (182). We don't know any firstprinciple argument leading to the values of the right-moving chemical potential $\mu_{R}$, the rightmoving temperature $T_{R}$ and the left-moving conformal weight $h_{L}$. We will deduce those values from matching the CFT absorption probability with the gravitational result.

In general, the weight (185) will be complex and real weight will not be integers. However, a curious fact, described in $[129,224]$, is that for any axisymmetric perturbation $(m=0)$ of any integer spin $s$ of the Kerr black hole, the conformal weight (185) is an integer

$$
h_{R}=1+l,
$$


where $l=0,1, \ldots$. One can generalize this result to any axisymmetric perturbation of any vacuum five-dimensional near-horizon geometry [224]. Counter-examples exist in higher dimensions and for black holes in AdS [129]. There is no microscopic accounting of this feature at present.

Throwing the scalar $\Phi_{\text {bulk }}$ at the black hole is dual to exciting the CFT by acting with the operator $\mathcal{O}$. Reemission is represented by the action of the Hermitian conjugate operator. Therefore, the absorption probability is related to the thermal CFT two-point function [209]

$$
G\left(t^{+}, t^{-}\right)=\left\langle\mathcal{O}^{\dagger}\left(t^{+}, t^{-}\right) \mathcal{O}(0)\right\rangle
$$

where $t^{ \pm}$are the coordinates of the left and right moving sectors of the CFT. At left and right temperatures $\left(T_{L}, T_{R}\right)$ and at chemical potentials $\left(\mu_{L}, \mu_{R}\right)$ an operator with conformal dimensions $\left(h_{L}, h_{R}\right)$ and charges $\left(q_{L}, q_{R}\right)$ has the two-point function

$$
G \sim(-1)^{h_{L}+h_{R}}\left(\frac{\pi T_{L}}{\sinh \left(\pi T_{L} t^{+}\right)}\right)^{2 h_{L}}\left(\frac{\pi T_{R}}{\sinh \left(\pi T_{R} t^{-}\right)}\right)^{2 h_{R}} e^{i q_{L} \mu_{L} t^{+}+i q_{R} \mu_{R} t^{-}},
$$

which is determined by conformal invariance. From Fermi's golden rule, the absorption cross section is $[53,106,160]$

$$
\begin{aligned}
\sigma_{\mathrm{abs}}\left(\omega_{L}, \omega_{R}\right) \sim & \int d t^{+} d t^{-} e^{-i \omega_{R} t^{-}-i \omega_{L} t^{+}}\left[G\left(t^{+}-i \epsilon, t^{-}-i \epsilon\right)\right. \\
& \left.-G\left(t^{+}+i \epsilon, t^{-}+i \epsilon\right)\right] .
\end{aligned}
$$

Performing the integral in (190), we obtain ${ }^{15}$

$$
\sigma_{\mathrm{abs}} \sim T_{L}^{2 h_{L}-1} T_{R}^{2 h_{R}-1}\left(e^{\pi \tilde{\omega}_{L}+\pi \tilde{\omega}_{R}} \pm e^{-\pi \tilde{\omega}_{L}-\pi \tilde{\omega}_{R}}\right)\left|\Gamma\left(h_{L}+i \tilde{\omega}_{L}\right)\right|^{2}\left|\Gamma\left(h_{R}+i \tilde{\omega}_{R}\right)\right|^{2},
$$

where

$$
\tilde{\omega}_{L}=\frac{\omega_{L}-q_{L} \mu_{L}}{2 \pi T_{L}}, \quad \tilde{\omega}_{R}=\frac{\omega_{R}-q_{R} \mu_{R}}{2 \pi T_{R}} .
$$

In order to compare the bulk computations to the CFT result (191), we must match the conformal weights and the reduced momenta $\left(\tilde{\omega}_{L}, \tilde{\omega}_{R}\right)$. The gravity result (178) agrees with the CFT result (191) if and only if we choose

$$
\begin{aligned}
& h_{L}=\frac{1}{2}+\beta-|s|, \quad h_{R}=\frac{1}{2}+\beta, \\
& \tilde{\omega}_{L}=\operatorname{Re}\left(q_{\mathrm{eff}}\right), \quad \tilde{\omega}_{R}=\frac{n}{2 \pi}-\operatorname{Re}\left(q_{\mathrm{eff}}\right) .
\end{aligned}
$$

The right conformal weight matches with (185), consistent with $S L(2, \mathbb{R})_{R}$ conformal invariance. The left conformal weight is natural for a spin $s$ field since $\left|h_{L}-h_{R}\right|=|s|$. The value for $\tilde{\omega}_{L}$ is consistent with the temperature (180) and chemical potential (181). Indeed, since the left-movers span the $\phi$ direction of the black hole, we have $\omega_{L}=m$. We then obtain

$$
\tilde{\omega}_{L}=\frac{2 m J+q_{e} Q^{3}}{r_{+}^{2}+a^{2}}=\operatorname{Re}\left(q_{\mathrm{eff}}\right)
$$

after using the value (175). The value of $\tilde{\omega}_{R}$ is fixed by the matching. It determines one constraint between $\omega_{R}, \mu_{R}$ and $T_{R}$. However, there is a subtlety in the above matching procedure. The conformal weights $h_{L}$ and $h_{R}$ depend on $m$ through $\beta$. This $m$ dependence cannot originate from

15 The two-point function (189) has a branch cut, and as a result, one must find a way to fix the choice of relative sign between the two exponentials in (191). The sign is fixed by matching the gravitational computation to be $-(-1)^{2 s}$, where $s$ is the spin of the corresponding field. 
$\omega_{L}=m$ since $\omega_{L}$ is introduced after the Fourier transform (190), while $h_{L}, h_{R}$ are already defined in (189). One way to introduce this $m$ dependence is to assume that there is a right-moving current algebra and that the dual operator $\mathcal{O}$ has the zero-mode charge $q_{R}=m$, which amounts to imposing the condition (182). (It is then also natural to assume that the chemical potential is $\mu_{R} \sim \Omega_{J}$, but the matching does not depend on any particular value for $\mu_{R}$ [53].) This justifies why a right-moving current algebra was assumed in the CFT. The dependence of the conformal weights in $q_{e}$ is similarly made possible thanks to the existence of the left-moving current with $q_{L}=q_{e}$. The matching is finally complete.

Now, let us notice that the matching conditions (193)-(194) are "democratic" in that the roles of angular momentum and electric charge are put on an equal footing, as noted in [79, 71]. One can then also obtain the conformal weights and reduced left and right frequencies $\tilde{\omega}_{L}, \tilde{\omega}_{R}$ using alternative CFT descriptions such as the $\mathrm{CFT}_{Q}$ with Virasoro algebra along the gauge field direction, and the mixed $S L(2, \mathbb{Z})$ family of CFTs. We can indeed rewrite (192) in the alternative form

$$
\tilde{\omega}_{L}=\frac{m T_{e}+q_{e} T_{\phi}}{2 \pi T_{\phi} T_{e}}=\frac{q_{\chi}-\mu_{L}^{\phi, Q} m}{2 \pi T_{L}^{Q}},
$$

where $T_{L}^{Q}=R_{\chi} T_{e}$ is the left-moving temperature of the $\mathrm{CFT}_{Q}, \mu_{L}^{\phi, Q}$ is the chemical potential defined in (144) and $q_{\chi}=R_{\chi} q_{e}$ is the probe electric charge in units of the Kaluza-Klein circle length. The identification of the right-moving sector is unchanged except that now $q_{R}=q_{e}$. One can trivially extend the matching with the $S L(2, \mathbb{Z})$ family of CFTs conjectured to describe the (near-)extremal Kerr-Newman black hole.

In summary, near-superradiant absorption probabilities of probes in the near-horizon region of near-extremal black holes are exactly reproduced by conformal field theory two-point functions. This shows the consistency of a CFT description (or multiple CFT descriptions in the case where several $U(1)$ symmetries are present) of part of the dynamics of near-extremal black holes. We expect that a general scattering theory around any near-extremal black-hole solution of (1) will also be consistent with a CFT description, as supported by all cases studied beyond the Kerr-Newman black hole $[106,73,242,71,80,46]$.

Finally, let us note finally that the dynamics of the CFTs dual to the Kerr-Newman geometry close to extremality can be further investigated by computing three-point correlation functions in the near-horizon geometry, as initiated in [40, 39].

\subsection{Microscopic accounting of superradiance}

We mentioned in Section 2.1 that extremal spinning black holes that do not admit a globallydefined timelike Killing vector spontaneously emit quanta in the range of frequencies (11). This quantum effect is related by detailed balance to the classical effect of superradiant wave emission, which occur in the same range of frequencies.

It has been argued that the bound (11) essentially follows from Fermi-Dirac statistics of the fermionic spin-carrying degrees of freedom in the dual two-dimensional CFT [121] (see also [132]). These arguments were made for specific black holes in string theory but one expects that they can be applied to generic extremal spinning black holes, at least qualitatively. Let us review these arguments here.

One starts with the assumption that extremal spinning black holes are modeled by a $2 d$ CFT, where the left and right sectors are coupled only very weakly. Therefore, the total energy and entropy are approximately the sum of the left and right energies and entropies. The state corresponding to an extremal spinning black hole is modeled as a filled Fermi sea on the right sector with zero entropy and a thermal state on the left sector, which accounts for the black-hole entropy. The right-moving fermions form a condensate of aligned spins $s=+1 / 2$, which accounts for the

Living Reviews in Relativity

http://www. livingreviews.org/lrr-2012-11 
macroscopic angular momentum. It is expected from details of emission rates in several parametric regimes that fermions are only present on the right sector, while bosons are present in both sectors [105, 106].

Superradiant spontaneous emission is then modeled as the emission of quanta resulting from interaction of a left and a right-moving mode. Using details of the model such as the fact that the Fermi energy should be proportional to the angular velocity $\Omega_{J}$, one can derive the bound (11). We refer the reader to [132] for further details. It would be interesting to better compare these arguments to the present setup, and to see how these arguments could be generalized to the description of the bound (12) for static extremal rotating black holes. Let us finally argue that the existence of a qualitative process of superradiant emission in these models further supports the conjecture that the dual theory to extremal black holes is a chiral limit of a $2 d$ CFT instead of a chiral CFT with no right-moving sector. 


\section{$6 \quad$ Hidden Symmetries of Non-Extremal Black Holes}

In Section 4 we described evidence showing that the asymptotic growth of states of extremal rotating or charged black holes is controlled by a chiral half of a two-dimensional CFT, at least in the semi-classical limit. We also reviewed in Section 5 how the near-horizon dynamics of probes can be reproduced by manipulating near-chiral CFT two-point functions in the near-extremal limit. These analyses strongly rely on the existence of a decoupled near-horizon geometry for all extremal or near-extremal black holes. Away from extremality, one cannot decouple the horizon from the surrounding geometry. Therefore, it is unclear whether any of the previous considerations will be useful in describing non-extremal geometries.

It might then come as a surprise that even away from extremality, conformal invariance is present in the dynamics of probe scalar fields around the Kerr black hole in a specific regime (at low energy and close enough to the black hole as we will make more precise below) [68]. In that regime, the probe scalar field equation can be written in a $S L(2, \mathbb{R}) \times S L(2, \mathbb{R})$ invariant fashion in a region close enough to the horizon. Such a local hidden symmetry is non-geometric but appears in the probe dynamics. The $2 \pi$ periodic identification of the azimuthal angle $\phi$ breaks globallyconformal symmetry. Using the properties of this representation of conformal invariance, one can then argue that the Kerr black hole is described by a CFT with specific left and right-moving temperatures [68]

$$
T_{L}=\frac{M^{2}}{2 \pi J}, \quad T_{R}=\frac{\sqrt{M^{4}-J^{2}}}{2 \pi J} .
$$

The well-known low-energy scattering amplitudes coincide with correlators of a two-dimensional CFT with these temperatures. Finally, quite remarkably, the entropy of the Kerr black hole is then reproduced by Cardy's formula if one assumes that the CFT has left and right-moving central charges equal to the value $c_{L}=c_{R}=12 \mathrm{~J}$, which matches with the value for the left-moving central charge (129) derived at extremality. ${ }^{16}$

These observations are consistent with the interpretation of a $2 d$ CFT dual to the Kerr black hole, but the existence of such a CFT is conjectural. For example, there is no known derivation of two Virasoro algebras with central charges $c_{L}=c_{R}=12 \mathrm{~J}$ from the non-extremal Kerr geometry. Asymptotic symmetry group methods are not directly applicable here because the horizon is not an isolated system. Therefore, it is unclear how these Virasoro algebras could be derived in Kerr. However, as argued in [68], the resulting picture shows a remarkable cohesiveness and only future research can prove or disprove such a CFT interpretation.

Given the successful generalization of the extremal Kerr/CFT correspondence to several independent extremal black hole/CFT correspondences in gravity coupled to matter, as we reviewed above, it is natural to test the ideas proposed in [68] to more general black holes than the Kerr geometry. First, hidden symmetry can be found around the non-extremal Reissner-Nordström black hole [81, 77] under the assumption that the gauge field can be understood as a KaluzaKlein gauge field, as done in the extremal case [160]. One can also generalize the analysis to the Kerr-Newman black hole $[263,74,78]$. In complete parallel with the existence of an $S L(2, \mathbb{Z})$ family of CFT descriptions, there is a class of hidden $S L(2, \mathbb{R}) \times S L(2, \mathbb{R})$ symmetries of the KerrNewman black hole related with $S L(2, \mathbb{Z})$ transformations [75]. What has not been noted in the literature so far is that each member of the $S L(2, \mathbb{Z})$ family of CFTs describes only probes with a fixed ratio of probe angular momentum to probe charge as we will discuss in detail in Section 6.4. Therefore, one needs a family of CFTs to fully describe the dynamics of low energy, low charge and low mass probes. Remarkably, for all cases where a hidden local conformal invariance can be described, the non-extremal black-hole entropy matches with Cardy's formula using the central

\footnotetext{
16 Note that at extremality $J=M^{2}$, so the central charge at extremality (129) could as well be written as $c_{L}=12 M^{2}$. However, away from extremality, matching the black hole entropy requires that the central charge be expressed in terms of the quantized charge $c_{L}=12 \mathrm{~J}$.
}

Living Reviews in Relativity

http://www. livingreviews.org//rr-2012-11 
charges $c_{R}=c_{L}$ and using the value $c_{L}$ in terms of the quantized conserved charges derived at extremality. Five-dimensional asymptotically-flat black holes were also discussed in [189, 80].

In attempting to generalize the hidden symmetry arguments to four-dimensional black holes in AdS one encounters an apparent obstruction, as we will discuss in Section 6.2. It is expected that hidden symmetries are present at least close to extremality, as illustrated by five-dimensional analogues [46]. However, the structure of the wave equation is more intricate far from extremality because of the presence of complex poles, which might have a role to play in microscopic models [102].

Quite surprisingly, one can also find a single copy of hidden $S L(2, \mathbb{R})$ symmetry around the Schwarzschild black hole [45], which turns out to be globally defined. As a consequence, no dual temperature can be naturally defined in that case. This hidden symmetry can be understood as a special case of a generalized notion of hidden conformal symmetry around the Kerr geometry [202]. At present, it is unclear how these hidden symmetries fit in the general picture of the Kerr/CFT correspondence since the derivations of the central charges of the CFT dual to Kerr, ReissnerNordström or Kerr-Newman black holes are done at extremality, which clearly cannot be done in the Schwarzschild case.

All arguments presented in the literature so far have been derived for a probe scalar field. It is not clear if any of these arguments can be generalized to higher-spin fields, and, if such, a generalization would give the same values for the left and right-moving CFT temperatures. It would certainly be interesting to understand whether this is a technical obstruction that can be overcome or whether it is a fundamental limitation in the CFT descriptions.

Hidden symmetries in asymptotically-flat spacetimes only appear in a region close enough to the black hole. It has been suggested that one deform the geometry far from the black hole such that hidden symmetries appear in the entire resulting geometry $[108,107]$. The resulting "subtracted" geometries are not asymptotically flat and are supported by additional matter fields $[108,107,101]$. The nature of these geometries and their role in the Kerr/CFT correspondence remains to be clarified. We will therefore not cover these constructions in this review.

In what follows, we present a summary of the derivation of the hidden symmetries of the KerrNewman black hole and we discuss their CFT interpretation. We will limit our presentation to the approach of [68] but we will generalize the discussion to the Kerr-Newman black hole, which contains several new interesting features. In particular, we will show that each member of the conjectured $S L(2, \mathbb{Z})$ family of CFTs controls part of the dynamics of low energy, low charge and low mass probes. We do not review the matching of absorption probabilities with CFT correlation functions. This matching is very similar to the analysis already performed in Section 5 at nearextremality and it follows from local conformal invariance. As noted in [68], the only difference is that in the present context the region close enough to the horizon is not geometrically a nearhorizon region, but it does not affect the discussion.

\subsection{Scalar wave equation in Kerr-Newman}

Let us first discuss probe scalar fields on the Kerr-Newman black hole. The Klein-Gordon equation for a charged massive spin 0 field of mass $\mu$ and charge $q_{e}$ was analyzed in Section 5.2. Expanding in eigenmodes and using the fact that the equation is separable, we have

$$
\Phi(t, r, \theta, \phi)=e^{-i \omega t+i m \phi} S(\theta) R(r) .
$$


The equations for the functions $S(\theta)$ and $R(r)$ were written in (158) and (159). Substituting $A_{a \omega, l, m}^{0}=K_{l}+a^{2}\left(\omega^{2}-\mu^{2}\right)$, the equations can be written in a convenient way as

$$
\begin{aligned}
& {\left[\frac{1}{\sin \theta} \partial_{\theta}\left(\sin \theta \partial_{\theta}\right)-\frac{m^{2}}{\sin ^{2} \theta}+a^{2}\left(\omega^{2}-\mu^{2}\right) \cos ^{2} \theta+K_{l}\right] S(\theta)=0,} \\
& {\left[\partial_{r}\left(\Delta \partial_{r}\right)+\frac{\alpha\left(r_{+}\right)^{2}}{\left(r-r_{+}\right)\left(r_{+}-r_{-}\right)}-\frac{\alpha\left(r_{-}\right)^{2}}{\left(r-r_{-}\right)\left(r_{+}-r_{-}\right)}-K_{l}+V(r)\right] R(r)=0,}
\end{aligned}
$$

where $\Delta(r)=\left(r-r_{+}\right)\left(r-r_{-}\right)$. The function $\alpha(r)$ is defined as

$$
\alpha(r)=\left(2 M r-Q^{2}\right) \omega-a m-Q r q_{e},
$$

and is evaluated either at $r_{+}$or $r_{-}$and

$$
V(r)=\left(\omega^{2}-\mu^{2}\right) r^{2}+2 \omega\left(M \omega-q_{e} Q\right) r-\omega^{2} Q^{2}+\left(2 M \omega-q_{e} Q\right)^{2} .
$$

These equations can be solved by Heun functions, which are not among the usual special functions. A solution can be found only numerically.

\subsection{Scalar wave equation in Kerr-Newman-AdS}

The equations for probe scalars fields on the Kerr-Newman-AdS black hole can be obtained straightforwardly. We consider only massless probes for simplicity. Using again the decomposition (197), the Klein-Gordon equation is decoupled into an angular equation

$$
\left[\frac{1}{\sin \theta} \partial_{\theta}\left(\sin \theta \Delta_{\theta} \partial_{\theta}\right)-\frac{m^{2} \Xi^{2}}{\sin ^{2} \theta \Delta_{\theta}}+\frac{2 m a \Xi \omega-a^{2} \omega^{2} \sin ^{2} \theta}{\Delta_{\theta}}+C_{l}\right] S(\theta)=0,
$$

and a radial equation

$$
\left[\partial_{r}\left(\Delta \partial_{r}\right)+\frac{\left[\omega\left(r^{2}+a^{2}\right)-m a \Xi-q_{e} Q_{e} r\right]^{2}}{\Delta_{r}}-C_{l}\right] R(r)=0,
$$

where $C_{l}$ is a separation constant and the various functions and parameters in the equations have been defined in Section 2.4.4. In the flat limit, Eqs. (198)-(199) are recovered with $K_{l}=$ $C_{l}+2 m a \omega-a^{2} \omega^{2}$.

The radial equation has a more involved form than the corresponding flat equation (199) due to the fact that $\Delta_{r}$ is a quartic instead of a quadratic polynomial in $r$; see (42). More precisely, the quartic polynomial $\Delta_{r}$ can be written as

$$
\Delta_{r}=l^{-2}\left(r-r_{+}\right)\left(r-r_{-}\right)\left(r-r_{c}\right)\left(r-r_{c}^{*}\right),
$$

where $r_{c}$ is a complex root. The radial equation is a general Heun's equation due to the presence of two conjugate complex poles in (203) in addition to the two real poles corresponding to the inner and outer horizons and the pole at infinity.

It has been suggested that all these poles have a role to play in the microscopic description of the AdS black hole [102]. It is an open problem to unravel the structure of the hidden symmetries, if any, of the full non-extremal radial equation (203). It has been shown that in the context of five-dimensional black holes, one can find hidden conformal symmetry in the near-horizon region close to extremality [46]. It is expected that one could similarly neglect the two complex poles in the near-horizon region of near-extremal black holes, but this remains to be checked in detail. ${ }^{17}$ Since much remains to be understood, we will not discuss AdS black holes further.

\footnotetext{
17 Alternatively, it was suggested in $[73,71]$ that one can describe the dynamics of the scalar field in the nearhorizon region using the truncated expansion of $\Delta_{r}(r)$ around $r_{+}$at second order. However, the resulting function $\Delta_{r}^{\text {trunc }}$ has, in addition to the pole $r_{+}$, a fake pole $r_{*}$, which is not associated with any geometric or thermodynamic feature of the solution. Therefore, the physical meaning of this truncation is unclear.
} 


\subsection{Near-region scalar-wave equation}

Let us go back to the scalar wave equation around the Kerr-Newman black hole. We will now study a particular range of parameters, where the wave equations simplify. We will assume that the wave has low energy and low mass as compared to the black hole mass and low electric charge as compared to the black hole charge,

$$
\omega M=O(\epsilon), \quad \mu M=O(\epsilon), \quad q_{e} Q=O(\epsilon),
$$

where $\epsilon \ll 1$. From these approximations, we deduce that $\omega a, \omega r_{+}, \omega Q$ and $\mu a=O(\epsilon)$ as well.

We will only look at a specific region of the spacetime - the "near region" - defined by

$$
\omega r=O(\epsilon), \quad \mu r=O(\epsilon) .
$$

Note that the near region is a distinct concept from the near-horizon region $r-r_{+} \ll M$. Indeed, for sufficiently small $\omega$ and $\mu$, the value of $r$ defined by the near region can be arbitrarily large.

Using the approximations (205), the wave equation greatly simplifies. It can be solved both in the near region and in the far region $r \gg M$ in terms of special functions. A complete solution can then be obtained by matching near and far solutions together along a surface in the matching region $M \ll r \ll \omega^{-1}$. As noted in [68], conformal invariance results from the freedom to locally choose the radius of the matching surface within the matching region.

More precisely, using (205), the angular equation (198) reduces to the standard Laplacian on the two-sphere

$$
\left[\frac{1}{\sin \theta} \partial_{\theta}\left(\sin \theta \partial_{\theta}\right)-\frac{m^{2}}{\sin ^{2} \theta}+K_{l}\right] S(\theta)=O\left(\epsilon^{2}\right) .
$$

The solutions $e^{i m \phi} S(\theta)$ are spherical harmonics and the separation constants are

$$
K_{l}=l(l+1)+O\left(\epsilon^{2}\right) .
$$

In the near region, the function $V(r)$ defined in (201) is very small, $V(r)=O\left(\epsilon^{2}\right)$. The near region scalar-wave equation can then be written as

$$
\left[\partial_{r}\left(\Delta \partial_{r}\right)+\frac{\alpha\left(r_{+}\right)^{2}}{\left(r-r_{+}\right)\left(r_{+}-r_{-}\right)}-\frac{\alpha\left(r_{-}\right)^{2}}{\left(r-r_{-}\right)\left(r_{+}-r_{-}\right)}-l(l+1)\right] R(r)=0,
$$

where $\alpha(r)$ has been defined in (200).

\subsection{Local $S L(2, \mathbb{R}) \times S L(2, \mathbb{R})$ symmetries}

We will now make explicit the local $S L(2, \mathbb{R}) \times S L(2, \mathbb{R})$ symmetries of the near-horizon scalar field equations (209). For this purpose it is convenient to define the "conformal" coordinates $\left(\omega^{ \pm}, y\right)$ defined in terms of coordinates $\left(t, r, \phi^{\prime}\right)$ by (see [68] and [210] for earlier relevant work)

$$
\begin{aligned}
\omega^{+} & =\sqrt{\frac{r-r_{+}}{r-r_{-}}} e^{2 \pi T_{R}\left(\phi^{\prime}-\Omega_{R} t\right)}, \\
\omega^{-} & =\sqrt{\frac{r-r_{+}}{r-r_{-}}} e^{2 \pi T_{L}\left(\phi^{\prime}-\Omega_{L} t\right)}, \\
y & =\sqrt{\frac{r_{+}-r_{-}}{r-r_{-}}} e^{\pi T_{L}\left(\phi^{\prime}-\Omega_{L} t\right)+\pi T_{R}\left(\phi^{\prime}-\Omega_{R} t\right)} .
\end{aligned}
$$

The change of coordinates is locally invertible if $\Delta \Omega=\Omega_{L}-\Omega_{R} \neq 0$. We choose the chirality $\Delta \Omega>0$, as it will turn out to match the chirality convention in the description of extremal black holes in Section 4.4. 
Several choices of coordinate $\phi^{\prime} \sim \phi^{\prime}+2 \pi$ will lead to independent $S L(2, \mathbb{R}) \times S L(2, \mathbb{R})$ symmetries. For the Kerr black hole, there is only one meaningful choice: $\phi^{\prime}=\phi$. For the ReissnerNordström black hole, we identify $\phi^{\prime}=\chi / R_{\chi}$, where $\chi$ is the Kaluza-Klein coordinate that allows one to lift the gauge field to higher dimensions, as done in Section 4.3. For the Kerr-Newman black hole, we use, in general, a coordinate system $\left(\phi^{\prime}, \chi^{\prime}\right) \sim\left(\phi^{\prime}, \chi^{\prime}+2 \pi\right) \sim\left(\phi^{\prime}+2 \pi, \chi^{\prime}\right)$ parameterized by a $S L(2, \mathbb{Z})$ transformation

$$
\begin{aligned}
& \phi^{\prime}=p_{1} \phi+p_{2} \chi / R_{\chi}, \\
& \chi^{\prime}=p_{3} \phi+p_{4} \chi / R_{\chi},
\end{aligned}
$$

with $p_{1} p_{4}-p_{2} p_{3}=1$ so that

$$
\begin{gathered}
\partial_{\phi^{\prime}}=p_{4} \partial_{\phi}-p_{3} R_{\chi} \partial_{\chi}, \\
\partial_{\chi^{\prime}}=-p_{2} \partial_{\phi}+p_{1} R_{\chi} \partial_{\chi} .
\end{gathered}
$$

Let us define locally the vector fields

$$
\begin{aligned}
H_{1} & =i \partial_{+} \\
H_{0} & =i\left(\omega^{+} \partial_{+}+\frac{1}{2} y \partial_{y}\right), \\
H_{-1} & =i\left(\omega^{+2} \partial_{+}+\omega^{+} y \partial_{y}-y^{2} \partial_{-}\right),
\end{aligned}
$$

and

$$
\begin{aligned}
\bar{H}_{1} & =i \partial_{-}, \\
\bar{H}_{0} & =i\left(\omega^{-} \partial_{-}+\frac{1}{2} y \partial_{y}\right), \\
\bar{H}_{-1} & =i\left(\omega^{-2} \partial_{-}+\omega^{-} y \partial_{y}-y^{2} \partial_{+}\right) .
\end{aligned}
$$

These vector fields obey the $S L(2, \mathbb{R})$ Lie bracket algebra,

$$
\left[H_{0}, H_{ \pm 1}\right]=\mp i H_{ \pm 1}, \quad\left[H_{-1}, H_{1}\right]=-2 i H_{0},
$$

and similarly for $\left(\bar{H}_{0}, \bar{H}_{ \pm 1}\right)$. Note that

$$
T_{L} \bar{H}_{0}+T_{R} H_{0}=\frac{i}{2 \pi} \partial_{\phi^{\prime}} .
$$

The $S L(2, \mathbb{R})$ quadratic Casimir is

$$
\begin{aligned}
\mathcal{H}^{2} & =\overline{\mathcal{H}}^{2}=-H_{0}^{2}+\frac{1}{2}\left(H_{1} H_{-1}+H_{-1} H_{1}\right) \\
& =\frac{1}{4}\left(y^{2} \partial_{y}^{2}-y \partial_{y}\right)+y^{2} \partial_{+} \partial_{-} .
\end{aligned}
$$

In terms of the coordinates $\left(r, t, \phi^{\prime}\right)$, the Casimir becomes

$$
\begin{aligned}
\mathcal{H}^{2}= & -\frac{r_{+}-r_{-}}{\left(r-r_{+}\right)\left(4 \pi T_{R}\right)^{2}}\left(\partial_{\phi^{\prime}}+\frac{T_{L}+T_{R}}{T_{L} \Delta \Omega}\left(\partial_{t}+\Omega_{R} \partial_{\phi^{\prime}}\right)\right)^{2} \\
& +\frac{r_{+}-r_{-}}{\left(r-r_{-}\right)\left(4 \pi T_{R}\right)^{2}}\left(\partial_{\phi^{\prime}}+\frac{T_{L}-T_{R}}{T_{L} \Delta \Omega}\left(\partial_{t}+\Omega_{R} \partial_{\phi^{\prime}}\right)\right)^{2}+\partial_{r} \Delta \partial_{r},
\end{aligned}
$$

where $\Delta(r)=\left(r-r_{+}\right)\left(r-r_{-}\right)$. 
We will now match the radial wave equation around the Kerr-Newman black hole in the near region (209) with the eigenvalue equation

$$
\mathcal{H}^{2} \Phi=l(l+1) \Phi .
$$

The scalar field has the following eigenvalues $\partial_{t} \Phi=-i \omega \Phi$ and $\partial_{\phi} \Phi=i m \Phi$. In the case where an electromagnetic field is present, one can perform the uplift (2) and consider the five-dimensional gauge field (6). In that case, the eigenvalue of the five-dimensional gauge field under $\partial_{\chi}$ is the electric charge $\partial_{\chi} \Phi=i q_{e} \Phi$. Let us denote the eigenvalue along $\partial_{\phi^{\prime}}$ as $i m^{\prime} \equiv i\left(p_{4} m-p_{3} q_{e} R_{\chi}\right)$. Eqs. (209) and (220) will match if and only if the two following equations are obeyed

$$
\alpha\left(r_{ \pm}\right)=\frac{r_{+}-r_{-}}{4 \pi T_{R}}\left(-m^{\prime}+\frac{T_{L} \pm T_{R}}{T_{L} \Delta \Omega}\left(\omega-\Omega_{R} m^{\prime}\right)\right),
$$

where $\alpha(r)$ has been defined in (200).

For simplicity, let us first discuss the case of zero probe charge $q_{e}=0$ and non-zero probe angular momentum $m \neq 0$. The matching equations then admit a unique solution

$$
\begin{aligned}
& \Omega_{R}=0, \quad \Omega_{L}=\frac{a}{2 M^{2}-Q^{2}}, \\
& T_{L}=\frac{2 M^{2}-Q^{2}}{4 \pi J}, \quad T_{R}=\frac{M\left(r_{+}-r_{-}\right)}{4 \pi J},
\end{aligned}
$$

upon choosing $\phi^{\prime}=\phi$ (and $\chi^{\prime}=\chi / R_{\chi}$ ). This shows in particular that the Kerr black hole has a hidden symmetry, as derived originally in [68].

For probes with zero angular momentum $m=0$, but electric charge $q_{e} \neq 0$, there is also a unique solution,

$$
\begin{aligned}
\Omega_{R} & =\frac{Q}{2 M R_{\chi}}, \quad \Omega_{L}=\frac{M Q}{\left(2 M^{2}-Q^{2}\right) R_{\chi}}, \\
T_{L} & =\frac{\left(2 M^{2}-Q^{2}\right) R_{\chi}}{2 \pi Q^{3}}, \quad T_{R}=\frac{M\left(r_{+}-r_{-}\right) R_{\chi}}{2 \pi Q^{3}},
\end{aligned}
$$

upon choosing $\phi^{\prime}=\chi / R_{\chi}$ (and $\chi^{\prime}=-\phi$ ). This shows, in particular, that the Reissner-Nordström black hole admits a hidden symmetry, as pointed out in [81, 77].

Finally, one can more generally solve the matching equation for any probe scalar field whose probe angular momentum and probe charge are related by

$$
p_{2} m-p_{1} q_{e} R_{\chi}=0 .
$$

In that case, one chooses the coordinate system (211) and the unique solution is then

$$
\begin{aligned}
& \Omega_{R}=\frac{p_{2} Q}{2 M R_{\chi}}, \quad \Omega_{L}=\frac{p_{1} a+p_{2} M Q / R_{\chi}}{2 M^{2}-Q^{2}}, \\
& T_{L}=\frac{2 M^{2}-Q^{2}}{2 \pi\left(2 p_{1} J+p_{2} Q^{3} / R_{\chi}\right)}, \quad T_{R}=\frac{M\left(r_{+}-r_{-}\right)}{2 \pi\left(2 p_{1} J+p_{2} Q^{3} / R_{\chi}\right)} .
\end{aligned}
$$

When $p_{1}=0$ and $Q \neq 0$ or $p_{2}=0$ and $J \neq 0$, one recovers the two previous particular cases. The condition (224) is equivalent to the fact that the scalar field has zero eigenvalue along $\partial_{\chi^{\prime}}$. Since $m$ and $q_{e} R_{\chi}$ are quantized, as derived in (7), there is always (at least) one solution to (224) with integers $p_{1}$ and $p_{2}$.

In conclusion, any low energy and low mass scalar probe in the near region (206) of the Kerr black hole admits a local hidden $S L(2, \mathbb{R}) \times S L(2, \mathbb{R})$ symmetry. Similarly, any low energy, low 
mass and low charge scalar probe in the near region (206) of the Reissner-Nordström black hole admits a local hidden $S L(2, \mathbb{R}) \times S L(2, \mathbb{R})$ symmetry. In the case of the Kerr-Newman black hole, we noticed that probes obeying $(205)$ also admit an $S L(2, \mathbb{R}) \times S L(2, \mathbb{R})$ hidden symmetry, whose precise realization depends on the ratio between the angular momentum and the electric charge of the probe. For a given ratio (224), hidden symmetries can be constructed using the coordinate $\phi^{\prime}=p_{1} \phi+p_{2} \chi / R_{\chi}$. Different choices of coordinate $\phi^{\prime}$ are relevant to describe different sectors of the low energy, low mass and low charge dynamics of scalar probes in the near region of the Kerr-Newman black hole. The union of these descriptions cover the entire dynamical phase space in the near region under the approximations (205)-(206).

\subsection{Symmetry breaking to $U(1)_{L} \times U(1)_{R}$}

The vector fields that generate the $S L(2, \mathbb{R}) \times S L(2, \mathbb{R})$ symmetries are not globally defined. They are not periodic under the angular identification

$$
\phi^{\prime} \sim \phi^{\prime}+2 \pi
$$

Therefore, the $S L(2, \mathbb{R})$ symmetries cannot be used to generate new global solutions from old ones. In other words, solutions to the wave equation in the near region do not form $S L(2, \mathbb{R}) \times S L(2, \mathbb{R})$ representations. In the $\left(\omega^{+}, \omega^{-}\right)$plane defined in $(210)$, the identification $(226)$ is generated by the $S L(2, \mathbb{R})_{L} \times S L(2, \mathbb{R})_{R}$ group element

$$
e^{-i 4 \pi^{2} T_{R} H_{0}-i 4 \pi^{2} T_{L} \bar{H}_{0}}
$$

as can be deduced from (217). This can be interpreted as the statement that the $S L(2, \mathbb{R})_{L} \times$ $S L(2, \mathbb{R})_{R}$ symmetry is spontaneously broken to the $U(1)_{L} \times U(1)_{R}$ symmetry generated by $\left(\bar{H}_{0}, H_{0}\right)$.

The situation is similar to the BTZ black hole in $2+1$ gravity that has a $S L(2, \mathbb{R})_{L} \times S L(2, \mathbb{R})_{R}$ symmetry, which is spontaneously broken by the identification of the angular coordinate. This breaking of symmetry can be interpreted in that case as placing the dual CFT to the BTZ black hole in a density matrix with left and right-moving temperatures dictated by the $S L(2, \mathbb{R})_{L} \times S L(2, \mathbb{R})_{R}$ group element generating the $2 \pi$ identification of the geometry [210].

In the case of non-extremal black-hole geometries, one can similarly interpret the symmetry breaking using a CFT as follows [68]. First, we need to assume that before the identification, the near region dynamics is described by a dual two-dimensional CFT, which possesses a ground state that is invariant under the full $S L(2, \mathbb{R})_{L} \times S L(2, \mathbb{R})_{R}$ symmetry. This is a strong assumption, since there are several (apparent) obstacles to the existence of a ground state, as we already discussed in the case of extremal black holes; see Section 4.4. Nevertheless, assuming the existence of this vacuum state, the two conformal coordinates $\left(\omega^{+}, \omega^{-}\right)$can be interpreted as the two null coordinates on the plane where the CFT vacuum state can be defined. At fixed $r$, the relation between conformal coordinates $\left(\omega^{+}, \omega^{-}\right)$and Boyer-Lindquist $(\phi, t)$ coordinates is, up to an $r$ dependent scaling,

$$
\omega^{ \pm}=e^{ \pm t^{ \pm}}
$$

where

$$
\begin{aligned}
& t^{+}=2 \pi T_{R}\left(\phi^{\prime}-\Omega_{R} t\right), \\
& t^{-}=-2 \pi T_{L}\left(\phi^{\prime}-\Omega_{L} t\right) .
\end{aligned}
$$

This is precisely the relation between Minkowski $\left(\omega^{ \pm}\right)$and Rindler $\left(t^{ \pm}\right)$coordinates. The periodic identification (226) then requires that the Rindler domain be restricted to a fundamental domain under the identification

$$
t^{+} \sim t^{+}+4 \pi^{2} T_{R}, \quad t^{-} \sim t^{-}-4 \pi^{2} T_{L}
$$


generated by the group element (227).

The quantum state describing this accelerating strip of Minkowski spacetime is obtained from the $S L(2, \mathbb{R})_{L} \times S L(2, \mathbb{R})_{R}$ invariant Minkowski vacuum by tracing over the quantum state in the region outside the strip. The result is a thermal density matrix at temperatures $\left(T_{L}, T_{R}\right)$. Hence, under the assumption of the existence of a CFT with a vacuum state, non-extremal black holes can be described as a finite temperature $\left(T_{L}, T_{R}\right)$ mixed state in a dual CFT.

It is familiar from the three-dimensional BTZ black hole that the identifications required to obtain extremal black holes are different than the ones required to obtain non-extremal black holes [27, 210]. Here as well, the vector fields (214)-(215) are not defined in the extremal limit because the change of coordinates (210) breaks down. Nevertheless, the extremal limit of the temperatures $T_{L}$ and $T_{R}$ match with the temperatures defined at extremality in Section 5.4. More precisely, the temperatures $T_{L}$ and $T_{R}$ defined in (222), (223) and (225) match with the temperatures defined at extremality $T_{\phi}, R_{\chi} T_{e}$ and $\left(p_{1} T_{\phi}^{-1}+p_{2}\left(R_{\chi} T_{e}\right)^{-1}\right)^{-1}$, respectively, where $T_{\phi}$ and $T_{e}$ are defined in (74). This is consistent with the interpretation that states corresponding to extremal black holes in the CFT can be defined as a limit of states corresponding to non-extremal black holes.

\subsection{Entropy matching}

We will now argue that the temperatures $T_{L}$ and $T_{R}$ obtained in Section 6 combined with the analysis at extremality in Section 4 lead to a (several) microscopic counting(s) of the black hole entropy of the Kerr, Reissner-Nordström and Kerr-Newman black holes.

Let us assume that there is a two-dimensional CFT $\left(\mathrm{CFT}_{J}\right)$ describing the Kerr black hole, a two-dimensional CFT $\left(\mathrm{CFT}_{Q}\right)$ describing the Reissner-Nordström black hole and a $S L(2, \mathbb{Z})$ family of two-dimensional CFTs $\left(\mathrm{CFT}_{\left(p_{1}, p_{2}, p_{3}\right)}, p_{1}, p_{2}, p_{2} \in \mathbb{Z}\right)$ describing the Kerr-Newman black hole. If these CFTs are dual to the black hole, the entropy is reproduced by Cardy's formula

$$
\mathcal{S}_{\mathrm{CFT}}=\frac{\pi^{2}}{3}\left(c_{L} T_{L}+c_{R} T_{R}\right),
$$

which is valid when $T_{L} \gg 1, T_{R} \gg 1$. As already mentioned in Section 4.4 and argued in [156], the regime $T_{L} \gg 1, T_{R} \gg 1$ is not a necessary condition for Cardy's formula to be valid if these CFTs have special properties such as admitting a long string picture, as reviewed in Section 3.3.

Let us discuss the values of the central charges. In a CFT, the difference $c_{R}-c_{L}$ is proportional to the diffeomorphism anomaly of the CFT $[188,187]$. One can then argue from diffeomorphism invariance that the two left and right sectors should have the same value for the central charge,

$$
c_{R}=c_{L} .
$$

We obtained the value $c_{L}$ at extremality in Section 4.3 and checked that Cardy's formula reproduces the extremal black-hole entropy. One way to uniquely fix the value $c_{L}$ away from extremality would consist in matching Cardy's formula (232) with the Kerr-Newman black-hole entropy

$$
\mathcal{S}_{\mathrm{KN}}(\mathcal{M}, \mathcal{J}, \mathcal{Q})=\pi\left(r_{+}^{2}+a^{2}\right)
$$

using (233) and the values for the temperatures derived in Section 6.4. Therefore, the matching of black-hole entropy is true by construction, which is clearly unsatisfactory. It would be more satisfactory to have an independent computation of $c_{L}$ away from extremality, but such a computation is currently not available.

However, the resulting central charge $c_{L}$ is, however, non-trivial. For the $\mathrm{CFT}_{J}$, we obtain $c_{L}=12 J$. For $\mathrm{CFT}_{Q}$, we have $c_{Q}=6 Q^{3} / R_{\chi}$ and for the $\mathrm{CFT}_{\left(p_{1}, p_{2}, p_{3}\right)}$, we find $c_{\left(p_{1}, p_{2}\right)}=$ $6\left(p_{1}(2 J)+p_{2} Q^{3} / R_{\chi}\right)$. Quite remarkably, these central charges are expressed solely in terms of 
quantized charges. They do not depend on the mass of the black hole. This is a non-trivial feature that has no explanation so far.

The presence of several CFTs dual to the Kerr-Newman black hole is curious but not inconsistent. Each CFT describes part of the low-energy dynamics of probe scalar fields and multiple CFTs are needed in order to reproduce the full dynamics for arbitrary ratios of the probe angular momentum to probe electric charge. Therefore, each CFT description has therefore a range of applicability away from extremality.

\section{Summary and Open Problems}

\subsection{Summary}

Let us summarize the key results that have been derived so far. Any extremal black hole containing a compact $U(1)$ axial symmetry admits a Virasoro algebra in its near-horizon geometry with a nontrivial central charge. The black-hole entropy is reproduced by a chiral half of Cardy's formula. This result is robust for any diffeomorphism-invariant theory and holds even including scalar and gauge field couplings and higher-derivative corrections. Moreover, if a $U(1)$ gauge field can be geometrized into a Kaluza-Klein vector in a higher-dimensional spacetime, a Virasoro algebra can be defined along the Kaluza-Klein compact $U(1)$ direction and all analysis goes through in a similar fashion as for the axial $U(1)$ symmetry. The deep similarity between the effects of rotation and electric charge can be understood from the fact that these charges are on a similar footing in the higher-dimensional geometry. When two $U(1)$ symmetries are present, one can mix up the compact directions using a modular transformation and the construction of Virasoro algebras can still be made.

Independent of these constructions, the scattering probabilities of probes around the nearextremal Kerr-Newman black hole can be reproduced near the superradiant bound by manipulating near-chiral thermal two-point functions of a two-dimensional CFT. The result extends straightforwardly to other asymptotically-flat or AdS black holes in various gravity theories. Finally away from extremality, hidden $S L(2, \mathbb{R}) \times S L(2, \mathbb{R})$ symmetries are present in some scalar probes around the Kerr-Newman black hole close enough to the horizon. We showed that several CFTs are required to account for the entire probe dynamics in the near region in the regime of small mass, small energy and small charge. This analysis does not extend straightforwardly to AdS black holes.

These results - obtained in gravity coupled to matter - are naturally accounted for by assuming that the microstates of asymptotically-flat black holes, at extremality and away from extremality, can be described by $2 d$ CFTs and that the microstates of asymptotically-AdS black holes at extremality can be described by chiral halves of $2 d$ CFTs. Scattering amplitudes and hidden symmetries are also accounted for by assuming that part of the dynamics of black holes can be mapped to the dynamics of these CFTs once they are suitably coupled to the exterior black-hole region. By consistency with the gravitational analysis, several CFT descriptions are available when several compact $U(1)$ symmetries are present. The existence of such CFTs is conjectural and only future research will tell how far these Kerr/CFT correspondences and their extensions can be made more precise.

A fair concluding remark would be that our understanding of the Kerr, Reissner-Nordström and Kerr-Newman black hole has increased over the last four years, but there is still a long road ahead of us to comprehend what these CFTs really are and what they are telling us about the nature of quantum black holes.

Living Reviews in Relativity

http: //www. livingreviews . org/lrr-2012-11 


\subsection{Set of open problems}

We close this review with a list of open problems. We hope that the interested reader will tackle them with the aim of shedding more light on the Kerr/CFT correspondence. We tried to order the problems with increasing difficulty but the evaluation is rough and highly subjective.

1. Hidden symmetries have been discussed so far for spin 0 probes. Discuss hidden symmetries for a probe gauge field or a probe graviton on Kerr or Kerr-Newman. Does one obtain the same temperatures $T_{L}$ and $T_{R}$ as in the scalar probe case?

2. A black hole in de Sitter spacetime can be extremal in the sense that its outer radius coincides with the cosmological horizon. The resulting geometry, called the rotating Narirai geometry, has many similarities with the near-horizon geometries of extremal black holes in flat spacetime or in AdS spacetime. The main difference is that the near-horizon geometry is a warped product of $d S_{2}$ with $S^{2}$ instead of $\mathrm{AdS}_{2}$ with $S^{2}$. It has been conjectured that these extremal black holes are dual to the chiral half of a Euclidean CFT [7]. Test the conjecture by generalizing all arguments of the Kerr/CFT correspondence to this cosmological setting.

3. Away from extremality, it is curious that the right-moving temperature is given by $T_{R}=$ $T_{H} / \Omega_{J}$ for the Kerr-Newman black hole. Account for this fact. Also, for all known asymptotically-flat extremal black holes in Einstein gravity coupled to matter, the product of the horizon areas of the inner and outer horizon can be expressed in terms of quantized charges $(J, Q, \ldots)$ and fundamental constants only $[195,103,106]$. Explain this feature from a fundamental perspective.

4. In the analysis of near-extremal superradiant scattering for any spin, we discarded the unstable modes that are below the Breitenlohner-Freedman bound. Such modes have imaginary $\beta$; see (177). Clarify the match between these modes and CFT expectations for the KerrNewman black hole.

5. The probe scalar wave equation in Kerr-Newman-AdS has two complex poles in addition to poles corresponding to the inner and outer horizon and infinity. This prevented a straightforward generalization of the hidden $S L(2, \mathbb{R}) \times S L(2, \mathbb{R})$ symmetry. Clarify the role of these additional poles. Also explain why the product of all horizon areas (inner, outer and complex horizons) seems in general not to depend on the mass of the black hole [102].

6. Near-horizon geometries of black-hole solutions of (1) have been classified. Classify the fourdimensional near-horizon geometries of extremal black holes for gravity coupled to charged scalars, massive vectors, $p$-forms and non-abelian gauge fields.

7. Compute the central charges $c_{L}$ and $c_{R}$ away from extremality. Also, compute the quantum corrections to the central charge $c_{L}$ and investigate the matching between the quantumcorrected entropy of extremal black holes derived in [241] and the asymptotic growth of states in the dual CFT.

8. Understand how the extension of the Kerr/CFT correspondence to extremal AdS black holes fits within the AdS/CFT correspondence. As discussed in [204], the extremal AdS-Kerr/CFT correspondence suggests that one can identify a non-trivial Virasoro algebra acting on the low-energy states of strongly coupled large $N$ super-Yang-Mills theory in an extremal thermal ensemble. Try to make this picture more precise.

9. From the point of view of $2 d$ CFTs, study if a $S L(2, \mathbb{Z})$ action exists that transforms a CFT into another CFT. This would clarify the existence of an $S L(2, \mathbb{Z})$ family of CFTs dual to the Kerr-Newman black hole. Note that this can be done for three-dimensional CFTs with a $U(1)$ current [267]. 
10. Compute the superradiant scattering amplitude of probe scalar fields on the Kerr-Newman geometry with first-order backreaction. Compare the result with the scattering amplitude defined in the CFT at one loop order (using two and three-point correlation functions).

11. Formulate a general scattering theory around near-extremal black-hole solutions of (1). This would require one to classify the geometries admiting a Killing-Yano tensor so that the wave equation could be separated. A long-standing problem already consists in separating and decoupling the wave e quation of a probe spin 1 or spin 2 field in the Kerr-Newman geometry.

12. Construct one example in string theory of an exact quantum field theory dual to (an embedding in string theory of) the Kerr black hole. Characterize whether that field theory is a CFT, a limit of a CFT, or a deformation thereof. 


\section{Acknowledgments}

This review originates from lectures given at Iberian Strings 2012 in Bilbao. I am very grateful to the organizers I. Bandos, I. Egusquiza, J.L. Mañes, M.A. Valle and C. Meliveo for the invitation to lecture in this outstanding and agreeable conference. I gratefully thank V. Balasubramanian, J. de Boer, B. Chen, C.-M. Chen, B. Chowdury, A. Castro, S. Detournay, J. Jottar, F. Larsen, S. Markoff, K. Murata, M. Rangamani, H. Reall, S. Sheikh-Jabbari, K. Skenderis, A. Strominger, A. Virmani and especially M. Guica and T. Hartman for interesting exchanges during the writing of this review. This work has been financially supported by the Nederlandse Organisatie voor Wetenschappelijk Onderzoek (NWO) via an NWO Vici grant. It is also currently supported by the FNRS, Belgium. I finally thank the organizers D. Berman, J. Conlon, N. Lambert, S. Mukhi and F. Quevedo of the program "Mathematics and Applications of Branes in String and M-theory" at the Isaac Newton Institute, Cambridge for support and hospitality during the final stages of this work. 


\section{References}

[1] Abbott, L.F. and Deser, S., "Stability of gravity with a cosmological constant", Nucl. Phys. B, 195, 76-96, (1982). [DOI]. (Cited on pages 33 and 38.)

[2] Adelberger, E.G., Heckel, B.R. and Nelson, A.E., "Tests of the Gravitational Inverse-Square Law", Annu. Rev. Nucl. Part. Sci., 53, 77-121, (2003). [DOI], [arXiv:hep-ph/0307284 [hep-ph]]. (Cited on page 8.)

[3] Agullo, I., Navarro-Salas, J., Olmo, G.J. and Parker, L., "Hawking radiation by Kerr black holes and conformal symmetry", Phys. Rev. Lett., 105, 211305, (2010). [DOI], [arXiv:1006.4404 [hep-th]]. (Cited on page 43.)

[4] Amsel, A.J., Horowitz, G.T., Marolf, D. and Roberts, M.M., "No dynamics in the extremal Kerr throat", J. High Energy Phys., 2009(09), 044, (2009). [DOI], [arXiv:0906.2376 [hep-th]]. (Cited on pages $23,25,26,27$, and 44.)

[5] Amsel, A.J., Marolf, D. and Roberts, M.M., "On the Stress Tensor of Kerr/CFT", J. High Energy Phys., 2009(10), 021, (2009). [DOI], [arXiv:0907.5023 [hep-th]]. (Cited on pages 34 and 37.)

[6] Andrade, T., Bañados, M., Benguria, R. and Gomberoff, A., "(2+1)-dimensional charged black hole in topologically massive electrodynamics", Phys. Rev. Lett., 95, 021102, (2005). [DOI], [hepth/0503095]. (Cited on page 16.)

[7] Anninos, D. and Hartman, T., "Holography at an extremal de Sitter horizon", J. High Energy Phys., 2010(03), 096, (2010). [DOI], [arXiv:0910.4587 [hep-th]]. (Cited on page 63.)

[8] Anninos, D., Li, W., Padi, M., Song, W. and Strominger, A., "Warped AdS 3 Black Holes", J. High Energy Phys., 2009(03), 130, (2009). [DOI], [arXiv:0807.3040 [hep-th]]. (Cited on page 16.)

[9] Arnowitt, R., Deser, S. and Misner, C.W., "Coordinate Invariance and Energy Expressions in General Relativity", Phys. Rev., 122, 997-1006, (1961). [DOI]. (Cited on pages 32 and 33.)

[10] Ashtekar, A., Beetle, C., Dreyer, O., Fairhurst, S., Krishnan, B., Lewandowski, J. and Wisniewski, J., "Generic Isolated Horizons and Their Applications", Phys. Rev. Lett., 85, 3564-3567, (2000). [DOI], [gr-qc/0006006]. (Cited on page 10.)

[11] Ashtekar, A., Beetle, C. and Fairhurst, S., "Isolated horizons: a generalization of black hole mechanics", Class. Quantum Grav., 16, L1-L7, (1999). [DOI], [arXiv:gr-qc/9812065 [gr-qc]]. (Cited on page 10.)

[12] Ashtekar, A., Bombelli, L. and Reula, O.A., "The covariant phase space of asymptotically flat gravitational fields", in Francaviglia, M. and Holm, D., eds., Mechanics, Analysis and Geometry: 200 Years after Lagrange, pp. 417-450, (North-Holland, Amsterdam; New York, 1991). (Cited on page 33.)

[13] Ashtekar, A and Hansen, R.O., "A unified treatment of null and spatial infinity in general relativity. I. Universal structure, asymptotic symmetries, and conserved quantities at spatial infinity", J. Math. Phys., 19, 1542-1566, (1978). [DOI]. (Cited on page 33.)

[14] Ashtekar, A. and Krishnan, B., "Isolated and Dynamical Horizons and Their Applications", Living Rev. Relativity, 7, lrr-2004-10, (2004). [arXiv:gr-qc/0407042]. URL (accessed 1 May 2012): http://www.livingreviews.org/lrr-2004-10. (Cited on page 10.)

[15] Ashtekar, A. and Magnon, A., "Asymptotically anti-de Sitter space-times", Class. Quantum Grav., 1, L39-L44, (1984). [DOI]. (Cited on page 33.)

[16] Ashtekar, A. and Romano, J.D., "Spatial infinity as a boundary of spacetime", Class. Quantum Grav., 9, 1069-1100, (1992). [DOI]. (Cited on page 33.) 
[17] Astefanesei, D., Goldstein, K., Jena, R.P., Sen, A. and Trivedi, S.P., "Rotating attractors", J. High Energy Phys., 2006(10), 058, (2006). [DOI], [arXiv:hep-th/0606244 [hep-th]]. (Cited on pages 14, 17, and 20.)

[18] Astefanesei, D., Goldstein, K. and Mahapatra, S., "Moduli and (un)attractor black hole thermodynamics", Gen. Relativ. Gravit., 40, 2069-2105, (2008). [DOI], [arXiv:hep-th/0611140 [hep-th]]. (Cited on page 14.)

[19] Astefanesei, D. and Yavartanoo, H., "Stationary black holes and attractor mechanism", Nucl. Phys. $B, \mathbf{7 9 4}, 13-27,(2008)$. [DOI], [arXiv:0706.1847 [hep-th]]. (Cited on page 15.)

[20] Azeyanagi, T., Compère, G., Ogawa, N., Tachikawa, Y. and Terashima, S., "Higher-Derivative Corrections to the Asymptotic Virasoro Symmetry of 4d Extremal Black Holes", Prog. Theor. Phys., 122, 355-384, (2009). [DOI], [arXiv:0903.4176 [hep-th]]. (Cited on pages 20, 23, and 38.)

[21] Azeyanagi, T., Ogawa, N. and Terashima, S., "Holographic Duals of Kaluza-Klein Black Holes", J. High Energy Phys., 2009(04), 061, (2009). [DOI], [arXiv:0811.4177 [hep-th]]. (Cited on pages 20, 35 , and 37.)

[22] Azeyanagi, T., Ogawa, N. and Terashima, S., "The Kerr/CFT Correspondence and String Theory", Phys. Rev. D, 79, 106009, (2009). [DOI], [arXiv:0812.4883 [hep-th]]. (Cited on pages 7 and 20.)

[23] Azeyanagi, T., Ogawa, N. and Terashima, S., "Emergent $\mathrm{AdS}_{3}$ in the Zero Entropy Extremal Black Holes", J. High Energy Phys., 2011(03), 004, (2011). [DOI], [arXiv:1010.4291 [hep-th]]. (Cited on pages 7,16 , and 30 .)

[24] Azeyanagi, T., Ogawa, N. and Terashima, S., "On Non-Chiral Extension of Kerr/CFT", J. High Energy Phys., 2011(06), 081, (2011). [DOI], [arXiv:1102.3423 [hep-th]]. (Cited on page 36.)

[25] Bañados, M., "Embeddings of the Virasoro algebra and black hole entropy", Phys. Rev. Lett., 82, 2030-2033, (1999). [DOI], [arXiv:hep-th/9811162 [hep-th]]. (Cited on page 30.)

[26] Bañados, M., Barnich, G., Compère, G. and Gomberoff, A., "Three-dimensional origin of Gödel spacetimes and black holes", Phys. Rev. D, 73, 044006, (2006). [DOI], [hep-th/0512105]. (Cited on page 16.)

[27] Bañados, M., Henneaux, M., Teitelboim, C. and Zanelli, J., "Geometry of the $(2+1)$ black hole", Phys. Rev., 48, 1506-1525, (1993). [DOI], [arXiv:gr-qc/9302012 [gr-qc]]. (Cited on page 61.)

[28] Bañados, M., Teitelboim, C. and Zanelli, J., "The black hole in three-dimensional spacetime", Phys. Rev. Lett., 69, 1849-1851, (1992). [DOI], [arXiv:hep-th/9204099 [hep-th]]. (Cited on page 40.)

[29] Balasubramanian, V., de Boer, J., Jejjala, V. and Simón, J., "Entropy of near-extremal black holes in $\mathrm{AdS}_{5}$ ", J. High Energy Phys., 2008(05), 067, (2008). [DOI], [arXiv:0707.3601 [hep-th]]. (Cited on page 16.)

[30] Balasubramanian, V., de Boer, J., Sheikh-Jabbari, M.M. and Simón, J., "What is a chiral 2d CFT? And what does it have to do with extremal black holes?", J. High Energy Phys., 2010(02), 017, (2010). [DOI], [arXiv:0906.3272 [hep-th]]. (Cited on pages 29, 36, and 40.)

[31] Balasubramanian, V., Parsons, J. and Ross, S.F., "States of a chiral 2d CFT", Class. Quantum Grav., 28, 045004, (2011). [DOI], [arXiv:1011.1803 [hep-th]]. (Cited on pages 7, 30, and 40.)

[32] Bardeen, J.M., Carter, B. and Hawking, S.W., "The Four Laws of Black Hole Mechanics", Commun. Math. Phys., 31, 161-170, (1973). [DOI]. Online version (accessed 13 June 2012): http://projecteuclid.org/euclid.cmp/1103858973. (Cited on page 5.)

[33] Bardeen, J.M. and Horowitz, G.T., "Extreme Kerr throat geometry: A vacuum analog of $A d S_{2} \times S^{2}$ ", Phys. Rev. D, 60, 104030, (1999). [DOI], [arXiv:hep-th/9905099 [hep-th]]. (Cited on pages 16 and 17.) 
[34] Bardeen, J.M., Press, W.H. and Teukolsky, S.A., "Rotating black holes: Locally nonrotating frames, energy extraction, and scalar syncrotron radiation", Astrophys. J., 178, 347-369, (1972). [DOI], [ADS]. (Cited on page 12.)

[35] Barnich, G. and Brandt, F., "Covariant theory of asymptotic symmetries, conservation laws and central charges", Nucl. Phys. B, 633, 3-82, (2002). [DOI], [arXiv:hep-th/0111246]. (Cited on pages 32, 37, and 38.)

[36] Barnich, G. and Compère, G., "Surface charge algebra in gauge theories and thermodynamic integrability", J. Math. Phys., 49, 042901, (2008). [DOI], [arXiv:0708.2378 [gr-qc]]. (Cited on pages 26, 32, 37, and 38.)

[37] Barnich, G. and Troessaert, C., "Symmetries of asymptotically flat 4 dimensional spacetimes at null infinity revisited", Phys. Rev. Lett., 105, 111103, (2010). [DOI], [arXiv:0909.2617 [gr-qc]]. (Cited on page 33.)

[38] Barnich, G. and Troessaert, C., "BMS charge algebra", J. High Energy Phys., 2011(12), 105, (2011). [DOI], [arXiv:1106.0213 [hep-th]]. (Cited on page 33.)

[39] Becker, M., Cremonini, S. and Schulgin, W., "Correlation Functions and Hidden Conformal Symmetry of Kerr Black Holes", J. High Energy Phys., 2010(09), 022, (2010). [DOI], [arXiv:1005.3571 [hep-th]]. (Cited on page 52.)

[40] Becker, M., Cremonini, S. and Schulgin, W., "Extremal Three-point Correlators in Kerr/CFT", J. High Energy Phys., 2011(02), 007, (2011). [DOI], [arXiv:1004.1174 [hep-th]]. (Cited on page 52.)

[41] Becker, M. and Schulgin, W., "Boundary Terms, Spinors and Kerr/CFT", J. High Energy Phys., 2012(04), 063, (2012). [DOI], [arXiv:1202.1528 [hep-th]]. (Cited on page 45.)

[42] Bekenstein, J.D., "Black holes and the second law", Lett. Nuovo Cimento, 4, 737-740, (1972). [DOI]. (Cited on page 5.)

[43] Bengtsson, I. and Sandin, P., "Anti de Sitter space, squashed and stretched", Class. Quantum Grav., 23, 971-986, (2006). [DOI], [arXiv:gr-qc/0509076 [gr-qc]]. (Cited on page 16.)

[44] Berti, E., Cardoso, V. and Casals, M., "Eigenvalues and eigenfunctions of spin-weighted spheroidal harmonics in four and higher dimensions", Phys. Rev. D, 73, 024013, (2006). [DOI], [arXiv:grqc/0511111 [gr-qc]]. (Cited on page 46.)

[45] Bertini, S., Cacciatori, S.L. and Klemm, D., "Conformal structure of the Schwarzschild black hole", Phys. Rev. D, 85, 064018, (2012). [DOI], [arXiv:1106.0999 [hep-th]]. (Cited on page 55.)

[46] Birkandan, T. and Cvetič, M., "Conformal Invariance and Near-extreme Rotating AdS Black Holes", Phys. Rev. D, 84, 044018, (2011). [DOI], [arXiv:1106.4329 [hep-th]]. (Cited on pages 43, 52, 55, and 56.)

[47] Birrell, N.D. and Davies, P.C.W., Quantum fields in curved space, Cambridge Monographs on Mathematical Physics, (Cambridge University Press, Cambridge; New York, 1982). [Google Books]. (Cited on page 22.)

[48] Blöte, H.W.J., Cardy, J.L. and Nightingale, M.P., "Conformal Invariance, the Central Charge, and Universal Finite-Size Amplitudes at Criticality", Phys. Rev. Lett., 56, 742-745, (1986). [DOI]. (Cited on page 29.)

[49] Bossard, G., "Octonionic black holes", J. High Energy Phys., 2012(05), 113, (2012). [DOI], [arXiv:1203.0530 [hep-th]]. (Cited on page 7.)

[50] Brady, P.R. and Chambers, C.M., "Nonlinear instability of Kerr-type Cauchy horizons", Phys. Rev., 51, 4177-4186, (1995). [DOI], [arXiv:gr-qc/9501025]. (Cited on page 13.) 
[51] Brady, P.R., Droz, S. and Morsink, S.M., "Late-time singularity inside nonspherical black holes", Phys. Rev. D, 58, 084034, (1998). [DOI], [arXiv:gr-qc/9805008 [gr-qc]]. (Cited on page 13.)

[52] Brady, P.R. and Smith, J.D., "Black Hole Singularities: A Numerical Approach", Phys. Rev. Lett., 75, 1256-1259, (1995). [DOI], [ADS], [arXiv:gr-qc/9506067 [gr-qc]]. (Cited on page 13.)

[53] Bredberg, I., Hartman, T., Song, W. and Strominger, A., "Black Hole Superradiance From Kerr/CFT", J. High Energy Phys., 2010(04), 019, (2010). [DOI], [arXiv:0907.3477 [hep-th]]. (Cited on pages $5,25,43,48,49,50,51$, and 52 .)

[54] Bredberg, I., Keeler, C., Lysov, V. and Strominger, A., "Lectures on the Kerr/CFT Correspondence", Nucl. Phys. B (Proc. Suppl.), 216, 194-210, (2011). [DOI], [arXiv:1103.2355 [hep-th]]. (Cited on pages 6 and 17.)

[55] Breitenlohner, P. and Freedman, D.Z., "Positive Energy in Anti-de Sitter Backgrounds and Gauged Extended Supergravity", Phys. Lett. B, 115, 197-201, (1982). [DOI]. (Cited on page 48.)

[56] Breitenlohner, P. and Freedman, D.Z., "Stability in Gauged Extended Supergravity", Ann. Phys. (N.Y.), 144, 249-281, (1982). [DOI]. (Cited on page 38.)

[57] Brenneman, L.W. and Reynolds, C.S., "Constraining Black Hole Spin Via X-ray Spectroscopy", Astrophys. J., 652, 1028-1043, (2006). [DOI], [arXiv:astro-ph/0608502 [astro-ph]]. (Cited on page 5.)

[58] Brown, J.D. and Henneaux, M., "Central Charges in the Canonical Realization of Asymptotic Symmetries: An example from three-dimensional gravity", Commun. Math. Phys., 104, 207-226, (1986). [DOI]. Online version (accessed 1 May 2012):

http://projecteuclid.org/euclid.cmp/1104114999. (Cited on pages 5, 33, 37, and 41.)

[59] Brown, J.D. and Henneaux, M., "On the Poisson brackets of differentiable generators in classical field theory", J. Math. Phys., 27, 489-491, (1986). [DOI]. (Cited on pages 32 and 37.)

[60] Callan, C.G. and Maldacena, J.M., "D-brane Approach to Black Hole Quantum Mechanics", Nucl. Phys. B, 472, 591-610, (1996). [DOI], [arXiv:hep-th/9602043]. (Cited on page 5.)

[61] Cardy, J.L., "Operator Content of Two-dimensional Conformally Invariant Theories", Nucl. Phys. $B$, 270, 186-204, (1986). (Cited on page 29.)

[62] Carlip, S., "What we don't know about BTZ black hole entropy", Class. Quantum Grav., 15, 36093625, (1998). [DOI], [hep-th/9806026]. (Cited on page 29.)

[63] Carlip, S., "Black hole entropy from conformal field theory in any dimension", Phys. Rev. Lett., 82, 2828-2831, (1999). [DOI], [hep-th/9812013]. (Cited on page 5.)

[64] Carneiro da Cunha, B. and de Queiroz, A.R., "Kerr-CFT from black-hole thermodynamics", J. High Energy Phys., 2010(08), 076, (2010). [DOI], [arXiv:1006.0510 [hep-th]]. (Cited on page 15.)

[65] Carter, B., "Global structure of the Kerr family of gravitational fields", Phys. Rev., 174, 1559-1571, (1968). [DOI]. (Cited on page 45.)

[66] Carter, B., "Black Hole Equilibrium States", in DeWitt, C. and DeWitt, B.S., eds., Black Holes, Based on lectures given at the 23rd session of the Summer School of Les Houches, 1972, pp. 57-214, (Gordon and Breach, New York, 1973). (Cited on page 10.)

[67] Castro, A. and Larsen, F., "Near extremal Kerr entropy from $\mathrm{AdS}_{2}$ quantum gravity", J. High Energy Phys., 2009(12), 037, (2009). [DOI], [arXiv:0908.1121 [hep-th]]. (Cited on pages 36 and 50.)

[68] Castro, A., Maloney, A. and Strominger, A., "Hidden Conformal Symmetry of the Kerr Black Hole", Phys. Rev. D, 82, 024008, (2010). [DOI], [arXiv:1004.0996 [hep-th]]. (Cited on pages 5, 54, 55, 57, 59, and 60.) 
[69] Chamblin, A., Emparan, R., Johnson, C.V. and Myers, R.C., "Charged AdS black holes and catastrophic holography", Phys. Rev. D, 60, 064018, (1999). [DOI], [arXiv:hep-th/9902170 [hep-th]]. (Cited on page 8.)

[70] Chandrasekhar, S., The Mathematical Theory of Black Holes, The International Series of Monographs on Physics, 69, (Clarendon, Oxford, 1983). [Google Books]. (Cited on page 45.)

[71] Chen, B., Chen, C.-M. and Ning, B., "Holographic Q-picture of Kerr-Newman-AdS-dS Black Hole", Nucl. Phys. B, 853, 196-209, (2011). [DOI], [arXiv:1010.1379 [hep-th]]. (Cited on pages 19, 42, 43, 52 , and 56.)

[72] Chen, B. and Chu, C.-S., "Real-Time Correlators in Kerr/CFT Correspondence", J. High Energy Phys., 2010(05), 004, (2010). [DOI], [arXiv:1001.3208 [hep-th]]. (Cited on page 43.)

[73] Chen, B. and Long, J., "On Holographic description of the Kerr-Newman-AdS-dS black holes", J. High Energy Phys., 2010(08), 065, (2010). [DOI], [arXiv:1006.0157 [hep-th]]. (Cited on pages 43, 52 , and 56.)

[74] Chen, B. and Long, J., "Real-time Correlators and Hidden Conformal Symmetry in Kerr/CFT Correspondence", J. High Energy Phys., 2010(06), 018, (2010). [DOI], [arXiv:1004.5039 [hep-th]]. (Cited on pages 43 and 54.)

[75] Chen, B. and Zhang, J.-j., "General Hidden Conformal Symmetry of 4D Kerr-Newman and 5D Kerr Black Holes", J. High Energy Phys., 2011(08), 114, (2011). [DOI], [arXiv:1107.0543 [hep-th]]. (Cited on page 54.)

[76] Chen, B. and Zhang, J.-j., "Novel CFT Duals for Extreme Black Holes", Nucl. Phys. B, 856, 449-474, (2012). [DOI], [arXiv:1106.4148 [hep-th]]. (Cited on pages 35 and 42.)

[77] Chen, C.-M., Huang, Y.-M., Sun, J.-R., Wu, M.-F. and Zou, S.-J., "On Holographic Dual of the Dyonic Reissner-Nordstrom Black Hole", Phys. Rev. D, 82, 066003, (2010). [DOI], [arXiv:1006.4092 [hep-th]]. (Cited on pages 39, 43, 54, and 59.)

[78] Chen, C.-M., Huang, Y.-M., Sun, J.-R., Wu, M.-F. and Zou, S.-J., "Twofold Hidden Conformal Symmetries of the Kerr-Newman Black Hole", Phys. Rev. D, 82, 066004, (2010). [DOI], [arXiv:1006.4097 [hep-th]]. (Cited on pages 42 and 54.)

[79] Chen, C.-M., Huang, Y.-M. and Zou, S.-J., "Holographic Duals of Near-extremal Reissner-Nordstrøm Black Holes", J. High Energy Phys., 2010(03), 123, (2010). [DOI], [arXiv:1001.2833 [hep-th]]. (Cited on pages 42,43 , and 52 .)

[80] Chen, C.-M., Kamali, V. and Setare, M.R., "Holographic Q-Picture of Black Holes in Five Dimensional Minimal Supergravity", arXiv, e-print, (2010). [arXiv:1011.4556 [hep-th]]. (Cited on pages 43, 52 , and 55.)

[81] Chen, C.-M. and Sun, J.-R., "Hidden Conformal Symmetry of the Reissner-Nordström Black Holes", J. High Energy Phys., 2010(08), 034, (2010). [DOI], [arXiv:1004.3963 [hep-th]]. (Cited on pages 43, 54, and 59.)

[82] Chen, C.-M. and Sun, J.-R., "Holographic Dual of the Reissner-Nordström Black Hole", J. Phys.: Conf. Ser., 330, 012009, (2011). [DOI], [arXiv:1106.4407 [hep-th]]. (Cited on page 42.)

[83] Chow, D.D.K., Cvetič, M., Lü, H. and Pope, C.N., "Extremal Black Hole/CFT Correspondence in (Gauged) Supergravities", Phys. Rev. D, 79, 084018, (2009). [DOI], [arXiv:0812.2918 [hep-th]]. (Cited on pages 20,21, 23, and 35.)

[84] Christodoulou, D. and Ruffini, R., "Reversible transformations of a charged black hole", Phys. Rev. $D, 4,3552-3555,(1971)$. [DOI]. (Cited on page 12.) 
[85] Chruściel, P.T. and Lopes Costa, J., "On uniqueness of stationary vacuum black holes", Asterisque, 321, 195-265, (2008). [arXiv:0806.0016 [gr-qc]]. (Cited on page 10.)

[86] Chruściel, P.T. and Wald, R.M., "Maximal hypersurfaces in asymptotically stationary space-times", Commun. Math. Phys., 163, 561-604, (1994). [DOI], [arXiv:gr-qc/9304009 [gr-qc]]. (Cited on page 10.)

[87] Chrzanowski, P.L., "Vector Potential and Metric Perturbations of a Rotating Black Hole", Phys. Rev. D, 11, 2042-2062, (1975). [DOI]. (Cited on page 45.)

[88] Clement, G. and Gal'tsov, D., "Conformal mechanics on rotating Bertotti-Robinson spacetime", Nucl. Phys. B, 619, 741-759, (2001). [DOI], [arXiv:hep-th/0105237 [hep-th]]. (Cited on page 17.)

[89] Cohen, J.M. and Kegeles, L.S., "Space-time perturbations", Phys. Lett. A, 54, 5-7, (1975). [DOI]. (Cited on page 45.)

[90] Compère, G., Symmetries and conservation laws in Lagrangian gauge theories with applications to the Mechanics of black holes and to Gravity in three dimensions, Ph.D. thesis, (Université libre de Bruxelles, Bruxelles, 2007). [arXiv:0708.3153 [hep-th]]. (Cited on pages 26, 32, and 38.)

[91] Compère, G., de Buyl, S., Jamsin, E. and Virmani, A., " $G_{2}$ dualities in $D=5$ supergravity and black strings", Class. Quantum Grav., 26, 125016, (2009). [DOI], [arXiv:0903.1645 [hep-th]]. (Cited on page 11.)

[92] Compère, G. and Dehouck, F., "Relaxing the Parity Conditions of Asymptotically Flat Gravity", Class. Quantum Grav., 28, 245016, (2011). [DOI], [arXiv:1106.4045 [hep-th]]. (Cited on page 33.)

[93] Compère, G. and Detournay, S., "Centrally extended symmetry algebra of asymptotically Gödel spacetimes", J. High Energy Phys., 2007(03), 098, (2007). [DOI], [arXiv:hep-th/0701039]. (Cited on page 16.)

[94] Compère, G. and Detournay, S., "Boundary conditions for spacelike and timelike warped $A d S_{3}$ spaces in topologically massive gravity", J. High Energy Phys., 2009(08), 092, (2009). [DOI], [arXiv:0906.1243 [hep-th]]. (Cited on page 50.)

[95] Compère, G. and Detournay, S., "Semi-classical central charge in topologically massive gravity", Class. Quantum Grav., 26, 012001, (2009). [DOI], [arXiv:0808.1911 [hep-th]]. (Cited on page 50.)

[96] Compère, G. and Marolf, D., "Setting the boundary free in AdS/CFT", Class. Quantum Grav., 25, 195014, (2008). [DOI], [arXiv:0805.1902 [hep-th]]. (Cited on pages 34 and 38.)

[97] Compère, G., Murata, K. and Nishioka, T., "Central Charges in Extreme Black Hole/CFT Correspondence", J. High Energy Phys., 2009(05), 077, (2009). [DOI], [arXiv:0902.1001 [hep-th]]. (Cited on pages $20,26,33,37,39$, and 40 .)

[98] Compère, G., Song, W. and Virmani, A., "Microscopics of Extremal Kerr from Spinning M5 Branes", J. High Energy Phys., 2011(10), 087, (2011). [DOI], [arXiv:1010.0685 [hep-th]]. (Cited on pages 7 and 30.)

[99] Copsey, K. and Horowitz, G.T., "Role of dipole charges in black hole thermodynamics", Phys. Rev. $D, \mathbf{7 3}, 024015,(2006)$. [DOI], [hep-th/0505278]. (Cited on page 11.)

[100] Coussaert, O. and Henneaux, M., "Self-dual solutions of $2+1$ Einstein gravity with a negative cosmological constant", arXiv, e-print, (1994). [arXiv:hep-th/9407181]. (Cited on pages 16 and 36.)

[101] Cvetič, M. and Gibbons, G.W., "Conformal Symmetry of a Black Hole as a Scaling Limit: A Black Hole in an Asymptotically Conical Box", arXiv, e-print, (2012). [arXiv:1201.0601 [hep-th]]. (Cited on page 55.) 
[102] Cvetič, M., Gibbons, G.W. and Pope, C.N., "Universal Area Product Formulae for Rotating and Charged Black Holes in Four and Higher Dimensions", Phys. Rev. Lett., 106, 121301, (2011). [DOI], [arXiv:1011.0008 [hep-th]]. (Cited on pages 55, 56, and 63.)

[103] Cvetič, M. and Larsen, F., "General rotating black holes in string theory: Grey body factors and event horizons", Phys. Rev. D, 56, 4994-5007, (1997). [DOI], [arXiv:hep-th/9705192 [hep-th]]. (Cited on page 63.)

[104] Cvetič, M. and Larsen, F., "Greybody factors for rotating black holes in four dimensions", Nucl. Phys. B, 506, 107-120, (1997). [DOI], [arXiv:hep-th/9706071 [hep-th]]. (Cited on page 5.)

[105] Cvetič, M. and Larsen, F., "Greybody factors for black holes in four-dimensions: Particles with spin", Phys. Rev. D, 57, 6297-6310, (1998). [DOI], [arXiv:hep-th/9712118 [hep-th]]. (Cited on page 53.)

[106] Cvetič, M. and Larsen, F., "Greybody Factors and Charges in Kerr/CFT", J. High Energy Phys., 2009(09), 088, (2009). [DOI], [arXiv:0908.1136 [hep-th]]. (Cited on pages 43, 48, 49, 51, 52, 53, and 63.)

[107] Cvetič, M. and Larsen, F., "Conformal Symmetry for Black Holes in Four Dimensions", arXiv, e-print, (2011). [arXiv:1112.4846 [hep-th]]. (Cited on pages 5 and 55.)

[108] Cvetič, M. and Larsen, F., "Conformal Symmetry for General Black Holes", J. High Energy Phys., 2012(02), 122, (2012). [DOI], [arXiv:1106.3341 [hep-th]]. (Cited on page 55.)

[109] Cvetič, M. et al., "Embedding AdS black holes in ten and eleven dimensions", Nucl. Phys. B, 558, 96-126, (1999). [DOI], [arXiv:hep-th/9903214 [hep-th]]. (Cited on page 8.)

[110] Dabholkar, A., "Microstates of non-supersymmetric black holes", Phys. Lett. B, 402, 53-58, (1997). [DOI], [arXiv:hep-th/9702050 [hep-th]]. (Cited on page 14.)

[111] Dabholkar, A., Sen, A. and Trivedi, S.P., "Black hole microstates and attractor without supersymmetry", J. High Energy Phys., 2007(01), 096, (2007). [DOI], [arXiv:hep-th/0611143 [hep-th]]. (Cited on page 14.)

[112] Dafermos, M., "The interior of charged black holes and the problem of uniqueness in general relativity", Commun. Pure Appl. Math., 58, 445-504, (2005). [DOI], [arXiv:gr-qc/0307013 [gr-qc]]. (Cited on page 13.)

[113] David, J.R., Mandal, G. and Wadia, S.R., "Microscopic formulation of black holes in string theory", Phys. Rep., 369, 549-686, (2002). [DOI], [arXiv:hep-th/0203048 [hep-th]]. (Cited on page 5.)

[114] de Boer, J., "Six-dimensional supergravity on $S^{3} \times A d S_{3}$ and 2 d conformal field theory", Nucl. Phys. $B, \mathbf{5 4 8}, 139-166,(1999)$. [DOI], [arXiv:hep-th/9806104 [hep-th]]. (Cited on page 30.)

[115] de Boer, J., Johnstone, M., Sheikh-Jabbari, M.M. and Simón, J., "Emergent IR dual 2d CFTs in charged AdS5 black holes", arXiv, e-print, (2011). [arXiv:1112.4664 [hep-th]]. (Cited on pages 7 and 30.)

[116] de Boer, J., Sheikh-Jabbari, M.M. and Simón, J., "Near Horizon Limits of Massless BTZ and Their CFT Duals", Class. Quantum Grav., 28, 175012, (2011). [DOI], [arXiv:1011.1897 [hep-th]]. (Cited on pages 7,16 , and 30.)

[117] Denef, F., "Les Houches Lectures on Constructing String Vacua", arXiv, e-print, (2008). [arXiv:0803.1194 [hep-th]]. (Cited on page 7.)

[118] Denef, F. and Moore, G.W., "Split states, entropy enigmas, holes and halos", J. High Energy Phys., 2011(11), 129, (2011). [DOI], [arXiv:hep-th/0702146 [HEP-TH]]. (Cited on pages 14 and 17.) 
[119] Detournay, S., Orlando, D., Petropoulos, P.M. and Spindel, P., "Three-dimensional black holes from deformed anti de Sitter", J. High Energy Phys., 2005(07), 072, (2005). [DOI], [hep-th/0504231]. (Cited on page 16.)

[120] Di Francesco, P., Mathieu, P. and Sénéchal, D., Conformal Field Theory, Graduate Texts in Contemporary Physics, (Springer, New York; Berlin, 1997). (Cited on page 28.)

[121] Dias, O.J.C., Emparan, R. and Maccarrone, A., "Microscopic theory of black hole superradiance", Phys. Rev. D, 77, 064018, (2008). [DOI], [arXiv:0712.0791 [hep-th]]. (Cited on pages 17 and 52.)

[122] Dias, O.J. C., Reall, H.S. and Santos, J.E., "Kerr-CFT and gravitational perturbations", J. High Energy Phys., 2009(08), 101, (2009). [DOI], [arXiv:0906.2380 [hep-th]]. (Cited on pages 26, 27, 44, and 45.)

[123] Dijkgraaf, R., "Instanton strings and hyperKahler geometry", Nucl. Phys. B, 543, 545-571, (1999). [DOI], [arXiv:hep-th/9810210 [hep-th]]. (Cited on page 30.)

[124] Dotti, G., Gleiser, R.J. and Ranea-Sandoval, I.F., "Instabilities in Kerr spacetimes", Int. J. Mod. Phys. E, 20, 27-31, (2011). [DOI], [arXiv:1111.5974 [gr-qc]]. (Cited on page 13.)

[125] Dotti, G., Gleiser, R.J., Ranea-Sandoval, I.F. and Vucetich, H., "Gravitational instabilities in Kerr space times", Class. Quantum Grav., 25, 245012, (2008). [DOI], [arXiv:0805.4306 [gr-qc]]. (Cited on page 13.)

[126] Dowker, F., Gauntlett, J.P., Giddings, S.B. and Horowitz, G.T., "On pair creation of extremal black holes and Kaluza-Klein monopoles", Phys. Rev. D, 50, 2662-2679, (1994). [DOI], [arXiv:hepth/9312172 [hep-th]]. (Cited on page 10.)

[127] Duff, M.J., Lü, H. and Pope, C.N., " $A d S_{3} \times S^{3}$ (un)twisted and squashed, and an $O(2,2, Z)$ multiplet of dyonic strings", Nucl. Phys. B, 544, 145-180, (1999). [DOI], [arXiv:hep-th/9807173 [hep-th]]. (Cited on page 16.)

[128] Duffy, G. and Ottewill, A.C., "The Renormalized stress tensor in Kerr space-time: Numerical results for the Hartle-Hawking vacuum", Phys. Rev. D, 77, 024007, (2008). [DOI], [arXiv:gr-qc/0507116 [gr-qc]]. (Cited on page 22.)

[129] Durkee, M. and Reall, H.S., "Perturbations of near-horizon geometries and instabilities of MyersPerry black holes", Phys. Rev. D, 83, 104044, (2011). [DOI], [arXiv:1012.4805 [hep-th]]. (Cited on pages 43,50 , and 51.)

[130] El-Showk, S. and Guica, M., "Kerr/CFT, dipole theories and nonrelativistic CFTs", arXiv, e-print, (2011). [arXiv:1108.6091 [hep-th]]. (Cited on pages 7, 28, and 30.)

[131] Emparan, R. and Horowitz, G.T., "Microstates of a Neutral Black Hole in M Theory", Phys. Rev. Lett., 97, 141601, (2006). [DOI], [arXiv:hep-th/0607023 [hep-th]]. (Cited on page 14.)

[132] Emparan, R. and Maccarrone, A., "Statistical description of rotating Kaluza-Klein black holes", Phys. Rev. D, 75, 084006, (2007). [DOI], [arXiv:hep-th/0701150 [hep-th]]. (Cited on pages 14, 52, and 53.)

[133] Emparan, R. and Reall, H.S., "A rotating black ring in five dimensions", Phys. Rev. Lett., 88, 101101, (2002). [DOI], [hep-th/0110260]. (Cited on page 17.)

[134] Fabian, A.C. et al., "Broad line emission from iron K- and L-shell transitions in the active galaxy 1H0707-495", Nature, 459, 540-542, (2009). [DOI], [ADS]. (Cited on page 5.)

[135] Fareghbal, R., Gowdigere, C.N., Mosaffa, A.E. and Sheikh-Jabbari, M.M., "Nearing extremal intersecting giants and new decoupled sectors in $N=4$ SYM", J. High Energy Phys., 2008(08), 070, (2008). [DOI], [arXiv:0801.4457 [hep-th]]. (Cited on page 16.) 
[136] Faulkner, T., Liu, H., McGreevy, J. and Vegh, D., "Emergent quantum criticality, Fermi surfaces, and $\mathrm{AdS}_{2}$ ", Phys. Rev. D, 83, 125002, (2011). [DOI], [arXiv:0907.2694 [hep-th]]. (Cited on page 27.)

[137] Fefferman, C. and Robin Graham, C., "Conformal Invariants", in Élie Cartan et les mathématiques d'aujourd'hui, The mathematical heritage of Elie Cartan, Lyon, 25-29 June 1984, Astérisque, p. 95, (Société mathématique de France, Paris, 1985). (Cited on page 40.)

[138] Fender, R.P., Gallo, E. and Russell, D., "No evidence for black hole spin powering of jets in Xray binaries", Mon. Not. R. Astron. Soc., 406, 1425-1434, (2010). [DOI], [ADS], [arXiv:1003.5516 [astro-ph.HE]]. (Cited on page 5.)

[139] Ferrara, S., Gibbons, G.W. and Kallosh, R., "Black holes and critical points in moduli space", Nucl. Phys. B, 500, 75-93, (1997). [DOI], [arXiv:hep-th/9702103 [hep-th]]. (Cited on page 14.)

[140] Ferrara, S. and Kallosh, R., "Supersymmetry and attractors", Phys. Rev. D, 54, 1514-1524, (1996). [DOI], [arXiv:hep-th/9602136 [hep-th]]. (Cited on page 14.)

[141] Ferrara, S., Kallosh, R. and Strominger, A., " $N=2$ extremal black holes", Phys. Rev., 52, 54125416, (1995). [DOI], [arXiv:hep-th/9508072]. (Cited on page 14.)

[142] Floyd, R., The Dynamics of Kerr Fields, Ph.D. thesis, (London University, London, 1973). (Cited on page 45.)

[143] Friedrich, H., Rácz, I. and Wald, R.M., "On the Rigidity Theorem for Spacetimes with a Stationary Event Horizon or a Compact Cauchy Horizon", Commun. Math. Phys., 204, 691-707, (1999). [DOI], [gr-qc/9811021]. (Cited on page 10.)

[144] Frolov, V.P. and Thorne, K.S., "Renormalized stress-energy tensor near the horizon of a slowly evolving, rotating black hole", Phys. Rev. D, 39, 2125-2154, (1989). [DOI]. (Cited on page 22.)

[145] Futterman, J.A.H., Handler, F.A. and Matzner, R.A., Scattering from Black Holes, Cambridge Monographs on Mathematical Physics, (Cambridge University Press, Cambridge, 1988). (Cited on page 44.)

[146] Garousi, M.R. and Ghodsi, A., "The RN/CFT Correspondence", Phys. Lett. B, 687, 79-83, (2010). [DOI], [arXiv:0902.4387 [hep-th]]. (Cited on page 39.)

[147] Geroch, R., "Structure of the Gravitational Field at Spatial Infinity", J. Math. Phys., 13, 956-968, (1972). [DOI]. (Cited on page 33.)

[148] Gibbons, G.W., Rietdijk, R.H. and van Holten, J.W., "SUSY in the sky", Nucl. Phys. B, 404, 42-64, (1993). [DOI], [arXiv:hep-th/9303112 [hep-th]]. (Cited on page 45.)

[149] Ginsparg, P.H., "Applied Conformal Field Theory", arXiv, e-print, (1988). [arXiv:hep-th/9108028 [hep-th]]. (Cited on page 28.)

[150] Goldstein, K., Iizuka, N., Jena, R.P. and Trivedi, S.P., "Non-supersymmetric attractors", Phys. Rev. D, 72, 124021, (2005). [DOI], [arXiv:hep-th/0507096 [hep-th]]. (Cited on page 14.)

[151] Goldstein, K. and Soltanpanahi, H., "CFT duals of black rings with higher derivative terms", Class. Quantum Grav., 29, 085016, (2012). [DOI], [arXiv:1108.4362 [hep-th]]. (Cited on pages 39 and 40.)

[152] Gou, L. et al., "The extreme spin of the black hole in Cygnus X-1", Astrophys. J., 742, 85, (2011). [DOI], [arXiv:1106.3690 [astro-ph.HE]]. (Cited on page 5.)

[153] Grana, M., "Flux compactifications in string theory: A Comprehensive review", Phys. Rep., 423, 91-158, (2006). [DOI], [arXiv:hep-th/0509003 [hep-th]]. (Cited on page 7.)

[154] Grumiller, D. and Piso, A.-M., "Exact relativistic viscous fluid solutions in near horizon extremal Kerr background", arXiv, e-print, (2009). [arXiv:0909.2041 [astro-ph.SR]]. (Cited on page 12.) 
[155] Gubser, S.S., Dynamics of D-brane black holes, Ph.D. thesis, (Princeton University, Princeton, 1998). [arXiv:hep-th/9908004 [hep-th]]. (Cited on page 5.)

[156] Guica, M., Hartman, T., Song, W. and Strominger, A., "The Kerr/CFT Correspondence", Phys. Rev. D, 80, 124008, (2009). [DOI], [arXiv:0809.4266 [hep-th]]. (Cited on pages 5, 17, 20, 21, 33, 34, $37,38,40,41$, and 61.)

[157] Guica, M. and Strominger, A., "Microscopic Realization of the Kerr/CFT Correspondence", J. High Energy Phys., 2011(02), 010, (2011). [DOI], [arXiv:1009.5039 [hep-th]]. (Cited on pages 7 and 30.)

[158] Gürses, M., "Perfect fluid sources in 2+1 dimensions", Class. Quantum Grav., 11, 2585-2587, (1994). [DOI]. (Cited on page 16.)

[159] Hartman, T., Murata, K., Nishioka, T. and Strominger, A., "CFT Duals for Extreme Black Holes", J. High Energy Phys., 2009(04), 019, (2009). [DOI], [arXiv:0811.4393 [hep-th]]. (Cited on pages 6, $8,19,20,21,33,34,35,37,38,39$, and 42.)

[160] Hartman, T., Song, W. and Strominger, A., "Holographic Derivation of Kerr-Newman Scattering Amplitudes for General Charge and Spin", J. High Energy Phys., 2010(03), 118, (2010). [DOI], [arXiv:0908.3909 [hep-th]]. (Cited on pages 43, 45, 48, 49, 50, 51, and 54.)

[161] Hawking, S.W., "Black holes in general relativity", Commun. Math. Phys., 25, 152-166, (1972). [DOI]. (Cited on page 10.)

[162] Hawking, S.W., "Particle creation by black holes", Commun. Math. Phys., 43, 199-220, (1975). [DOI]. (Cited on pages 5 and 12.)

[163] Hawking, S.W. and Ellis, G.F.R., The Large Scale Structure of Space-Time, Cambridge Monographs on Mathematical Physics, (Cambridge University Press, Cambridge, 1973). [Google Books]. (Cited on page 10.)

[164] Hawking, S.W. and Horowitz, G.T., "The gravitational Hamiltonian, action, entropy and surface terms", Class. Quantum Grav., 13, 1487-1498, (1996). [DOI], [gr-qc/9501014]. (Cited on page 38.)

[165] Hawking, S.W. and Reall, H.S., "Charged and rotating AdS black holes and their CFT duals", Phys. Rev. D, 61, 024014, (2000). [DOI], [arXiv:hep-th/9908109 [hep-th]]. (Cited on page 11.)

[166] Hayashi, H. and Tai, T.-S., " $R^{2}$ Correction to BMPV Black Hole Entropy from Kerr/CFT Correspondence", Phys. Lett. B, 710, 352-357, (2012). [DOI], [arXiv:1112.5417 [hep-th]]. (Cited on page 39.)

[167] Henneaux, M., "Asymptotically anti-de Sitter universes in d = 3, 4 and higher dimensions", in Ruffini, R., ed., Proceedings of the Fourth Marcel Grossmann Meeting on General Relativity, Held at the University of Rome La Sapienza, Rome, 17-21 June 1985, pp. 959-966, (North-Holland, Amsterdam; New York, 1986). (Cited on page 33.)

[168] Henneaux, M. and Teitelboim, C., "Asymptotically anti-de Sitter spaces", Commun. Math. Phys., 98, 391-424, (1985). [DOI]. Online version (accessed 1 May 2012): http://projecteuclid.org/euclid.cmp/1103942446. (Cited on page 33.)

[169] Hofman, D.M. and Strominger, A., "Chiral Scale and Conformal Invariance in 2D Quantum Field Theory", Phys. Rev. Lett., 107, 161601, (2011). [DOI], [arXiv:1107.2917 [hep-th]]. (Cited on page 28.)

[170] Hollands, S. and Ishibashi, A., "On the 'Stationary Implies Axisymmetric' Theorem for Extremal Black Holes in Higher Dimensions", Commun. Math. Phys., 291, 403-441, (2009). [DOI], [arXiv:0809.2659 [gr-qc]]. (Cited on pages 10 and 26.) 
[171] Hollands, S., Ishibashi, A. and Wald, R.M., "A Higher Dimensional Stationary Rotating Black Hole Must be Axisymmetric", Commun. Math. Phys., 271, 699-722, (2007). [DOI], [gr-qc/0605106]. (Cited on page 10.)

[172] Horowitz, G.T., Lowe, D.A. and Maldacena, J.M., "Statistical Entropy of Nonextremal FourDimensional Black Holes and U Duality", Phys. Rev. Lett., 77, 430-433, (1996). [DOI], [arXiv:hepth/9603195 [hep-th]]. (Cited on page 14.)

[173] Isono, H., Tai, T.-S. and Wen, W.-Y., "Kerr/CFT correspondence and five-dimensional BMPV black holes", Int. J. Mod. Phys. A, 24, 5659-5668, (2009). [DOI], [arXiv:0812.4440 [hep-th]]. (Cited on pages 20 and 35.)

[174] Israël, D., Kounnas, C., Orlando, D. and Petropoulos, P.M., "Electric/magnetic deformations of $S^{3}$ and $\mathrm{AdS}_{3}$, and geometric cosets", Fortschr. Phys., 53, 73-104, (2005). [DOI], [hep-th/0405213]. (Cited on page 16.)

[175] Israël, D., Kounnas, C. and Petropoulos, P.M., "Superstrings on NS5 backgrounds, deformed AdS 3 and holography", J. High Energy Phys., 2003(10), 028, (2003). [DOI], [arXiv:hep-th/0306053]. (Cited on page 16.)

[176] Iyer, V. and Wald, R.M., "Some properties of Noether charge and a proposal for dynamical black hole entropy", Phys. Rev., 50, 846-864, (1994). [DOI], [gr-qc/9403028]. (Cited on page 20.)

[177] Jacobson, T.A., "Introductory Lectures on Black Hole Thermodynamics", lecture notes, University of Maryland, (1996). URL (accessed 26 April 2012):

http://www.physics.umd.edu/grt/taj/776b/. (Cited on page 12.)

[178] Jacobson, T.A., Kang, G. and Myers, R.C., "On black hole entropy", Phys. Rev., 49, 6587-6598, (1994). [DOI], [arXiv:gr-qc/9312023]. (Cited on page 20.)

[179] Kallosh, R.E., "New attractors", J. High Energy Phys., 2005(12), 022, (2005). [DOI], [arXiv:hepth/0510024 [hep-th]]. (Cited on page 14.)

[180] Kang, G., Koga, J. and Park, M.-I., "Near horizon conformal symmetry and black hole entropy in any dimension", Phys. Rev. D, 70, 024005, (2004). [DOI], [arXiv:hep-th/0402113 [hep-th]]. (Cited on page 5.)

[181] Kaplan, D.M., Lowe, D.A., Maldacena, J.M. and Strominger, A., "Microscopic entropy of $N=2$ extremal black holes", Phys. Rev. D, 55, 4898-4902, (1997). [DOI], [arXiv:hep-th/9609204 [hep-th]]. (Cited on page 14.)

[182] Kato, S., "Mass and Spin of GRS 1915+105 Based on a Resonance Model of QPOs", Publ. Astron. Soc. Japan, 56, L25-L28, (2004). [ADS], [arXiv:astro-ph/0407251]. (Cited on page 6.)

[183] Kay, B.S. and Wald, R.M., "Theorems on the Uniqueness and Thermal Properties of Stationary, Nonsingular, Quasifree States on Space-Times with a Bifurcate Killing Horizon", Phys. Rep., 207, 49-136, (1991). [DOI], [ADS]. (Cited on page 23.)

[184] Kim, S.P. and Page, D.N., "Schwinger pair production in $d S_{2}$ and $A d S_{2}$ ", Phys. Rev. D, 78, 103517, (2008). [DOI], [arXiv:0803.2555 [hep-th]]. (Cited on page 48.)

[185] Kim, S.-S., Lindman Hörnlund, J., Palmkvist, J. and Virmani, A., "Extremal solutions of the $\mathrm{S}^{3}$ model and nilpotent orbits of $\mathrm{G}_{2}(2)$ ", J. High Energy Phys., 2010(08), 072, (2010). [DOI], [arXiv:1004.5242 [hep-th]]. (Cited on page 8.)

[186] Klemm, A. and Schmidt, M.G., "Orbifolds by cyclic permutations of tensor product conformal field theories", Phys. Lett. B, 245, 53-58, (1990). [DOI]. (Cited on page 30.) 
[187] Kraus, P., "Lectures on Black Holes and the $\mathrm{AdS}_{3} / \mathrm{CFT}_{2}$ Correspondence", in Bellucci, S., ed., Supersymmetric Mechanics - Vol. 3: Attractors and Black Holes in Supersymmetric Gravity, INFN Frascati Winter School, Lecture Notes in Physics, 755, pp. 193-247, (Springer, Berlin; New York, 2008). [DOI], [arXiv:hep-th/0609074 [hep-th]]. (Cited on page 61.)

[188] Kraus, P. and Larsen, F., "Holographic gravitational anomalies", J. High Energy Phys., 2006(01), 022, (2006). [DOI], [arXiv:hep-th/0508218]. (Cited on page 61.)

[189] Krishnan, C., "Hidden Conformal Symmetries of Five-Dimensional Black Holes", J. High Energy Phys., 2010(07), 039, (2010). [DOI], [arXiv:1004.3537 [hep-th]]. (Cited on page 55.)

[190] Krishnan, C. and Kuperstein, S., "A Comment on Kerr-CFT and Wald Entropy", Phys. Lett. B, 677, 326-331, (2009). [DOI], [arXiv:0903.2169 [hep-th]]. (Cited on page 38.)

[191] Kunduri, H.K., "Electrovacuum Near-horizon Geometries in Four and Five Dimensions", Class. Quantum Grav., 28, 114010, (2011). [DOI], [arXiv:1104.5072 [hep-th]]. (Cited on page 26.)

[192] Kunduri, H.K. and Lucietti, J., "A classification of near-horizon geometries of extremal vacuum black holes", J. Math. Phys., 50, 082502, (2009). [DOI], [arXiv:0806.2051 [hep-th]]. (Cited on page 26.)

[193] Kunduri, H.K. and Lucietti, J., "Uniqueness of near-horizon geometries of rotating extremal AdS(4) black holes", Class. Quantum Grav., 26, 055019, (2009). [DOI], [arXiv:0812.1576 [hep-th]]. (Cited on page 26.)

[194] Kunduri, H.K., Lucietti, J. and Reall, H.S., "Near-horizon symmetries of extremal black holes", Class. Quantum Grav., 24, 4169-4190, (2007). [DOI], [arXiv:0705.4214 [hep-th]]. (Cited on pages 13, 15, and 17.)

[195] Larsen, F., "A String model of black hole microstates", Phys. Rev. D, 56, 1005-1008, (1997). [DOI], [arXiv:hep-th/9702153 [hep-th]]. (Cited on page 63.)

[196] Lee, J. and Wald, R.M., "Local symmetries and constraints", J. Math. Phys., 31, 725-743, (1990). [DOI]. (Cited on page 32.)

[197] Long, J.C. and Price, J.C., "Current short range tests of the gravitational inverse square law", $C$. R. Physique, 4, 337-346, (2003). [DOI], [arXiv:hep-ph/0303057 [hep-ph]]. (Cited on page 8.)

[198] Lopes Cardoso, G., de Wit, B., Käppeli, J. and Mohaupt, T., "Stationary BPS solutions in $N=2$ supergravity with $R^{2}$-interactions", J. High Energy Phys., 2000(12), 019, (2000). [DOI], [arXiv:hepth/0009234 [hep-th]]. (Cited on page 14.)

[199] Lopes Cardoso, G., de Wit, B. and Mohaupt, T., "Corrections to macroscopic supersymmetric black hole entropy", Phys. Lett. B, 451, 309-316, (1999). [DOI], [arXiv:hep-th/9812082 [hep-th]]. (Cited on page 14.)

[200] Lopes Cardoso, G., de Wit, B. and Mohaupt, T., "Macroscopic entropy formulae and nonholomorphic corrections for supersymmetric black holes", Nucl. Phys. B, 567, 87-110, (2000). [DOI], [arXiv:hepth/9906094 [hep-th]]. (Cited on page 14.)

[201] Loran, F. and Soltanpanahi, H., "5D Extremal Rotating Black Holes and CFT duals", Class. Quantum Grav., 26, 155019, (2009). [DOI], [arXiv:0901.1595 [hep-th]]. (Cited on page 35.)

[202] Lowe, D.A. and Skanata, A., "Generalized hidden Kerr/CFT", arXiv, e-print, (2011). [arXiv:1112.1431 [hep-th]]. (Cited on page 55.)

[203] Lü, H., Mei, J. and Pope, C.N., "Kerr/CFT Correspondence in Diverse Dimensions", J. High Energy Phys., 2009(04), 054, (2009). [DOI], [arXiv:0811.2225 [hep-th]]. (Cited on pages 17, 20, and 35.) 
[204] Lü, H., Mei, J., Pope, C.N. and Vázquez-Poritz, J.F., "Extremal static AdS black hole/CFT correspondence in gauged supergravities", Phys. Lett. B, 673, 77-82, (2009). [DOI], [arXiv:0901.1677 [hep-th]]. (Cited on pages 8, 20,35, 40, and 63.)

[205] Maldacena, J.M., "The large $N$ limit of superconformal field theories and supergravity", Adv. Theor. Math. Phys., 2, 231-252, (1998). [arXiv:hep-th/9711200 [hep-th]]. (Cited on pages 5 and 7.)

[206] Maldacena, J.M., "The Large- $N$ Limit of Superconformal Field Theories and Supergravity", Adv. Theor. Math. Phys., 2, 231-252, (1998). [DOI], [arXiv:hep-th/9711200 [hep-th]]. (Cited on pages 30 and 41.)

[207] Maldacena, J.M., Michelson, J. and Strominger, A., "Anti-de Sitter fragmentation", J. High Energy Phys., 1999(02), 011, (1999). [DOI], [arXiv:hep-th/9812073 [hep-th]]. (Cited on pages 25 and 27.)

[208] Maldacena, J.M. and Strominger, A., "Black hole greybody factors and D-brane spectroscopy", Phys. Rev. D, 55, 861-870, (1997). [DOI], [arXiv:hep-th/9609026 [hep-th]]. (Cited on page 43.)

[209] Maldacena, J.M. and Strominger, A., "Universal low-energy dynamics for rotating black holes", Phys. Rev. D, 56, 4975-4983, (1997). [DOI], [arXiv:hep-th/9702015 [hep-th]]. (Cited on pages 5, 43, and 51.)

[210] Maldacena, J.M. and Strominger, A., " $A d S_{3}$ black holes and a stringy exclusion principle", J. High Energy Phys., 1998(12), 005, (1998). [DOI], [arXiv:hep-th/9804085]. (Cited on pages 57, 60, and 61.)

[211] Maldacena, J.M. and Susskind, L., "D-branes and fat black holes", Nucl. Phys. B, 475, 679-690, (1996). [DOI], [arXiv:hep-th/9604042 [hep-th]]. (Cited on page 31.)

[212] Marolf, D., "The dangers of extremes", Gen. Relativ. Gravit., 42, 2337-2343, (2010). [DOI], [arXiv:1005.2999 [gr-qc]]. (Cited on page 13.)

[213] Marolf, D. and Ross, S.F., "Boundary conditions and new dualities: Vector fields in AdS/CFT", J. High Energy Phys., 2006(11), 085, (2006). [DOI], [hep-th/0606113]. (Cited on page 38.)

[214] Matsuo, Y. and Nishioka, T., "New Near Horizon Limit in Kerr/CFT", J. High Energy Phys., 2010(12), 073, (2010). [DOI], [arXiv:1010.4549 [hep-th]]. (Cited on page 36.)

[215] Matsuo, Y., Tsukioka, T. and Yoo, C.-M., "Another Realization of Kerr/CFT Correspondence", Nucl. Phys. B, 825, 231-241, (2010). [DOI], [arXiv:0907.0303 [hep-th]]. (Cited on page 36.)

[216] Matsuo, Y., Tsukioka, T. and Yoo, C.-M., "Yet Another Realization of Kerr/CFT Correspondence", Europhys. Lett., 89, 60001, (2010). [DOI], [arXiv:0907.4272 [hep-th]]. (Cited on page 36.)

[217] McAllister, L. and Silverstein, E., "String Cosmology: A Review", Gen. Relativ. Gravit., 40, 565605, (2008). [DOI], [arXiv:0710.2951 [hep-th]]. (Cited on page 7.)

[218] McClintock, J.E., Shafee, R., Narayan, R., Remillard, R.A., Davis, S.W. et al., "The Spin of the NearExtreme Kerr Black Hole GRS 1915+105”, Astrophys. J., 652, 518-539, (2006). [DOI], [arXiv:astroph/0606076 [astro-ph]]. (Cited on page 5.)

[219] McClintok, J.E. and Remillard, R.A., "Measuring the Spins of Stellar-Mass Black Holes", in Astro2010: The Astronomy and Astrophysics Decadal Survey - Science White Papers, (National Academy of Sciences, Washington, DC, 2009). [ADS], [arXiv:0902.3488 [astro-ph.HE]]. URL (accessed 13 June 2012):

http://sites.nationalacademies.org/bpa/BPA_050603. (Cited on page 6.)

[220] McNamara, J.M., "Instability of Black Hole Inner Horizons", Proc. R. Soc. London, Ser. A, 358, 499-517, (1978). [DOI]. (Cited on page 13.)

[221] Mei, J., "The Entropy for General Extremal Black Holes", J. High Energy Phys., 2010(04), 005, (2010). [DOI], [arXiv:1002.1349 [hep-th]]. (Cited on page 7.) 
[222] Moussa, K.A., Clément, G., Guennoune, H. and Leygnac, C., "Three-dimensional Chern-Simons black holes", Phys. Rev. D, 78, 064065, (2008). [DOI], [arXiv:0807.4241 [gr-qc]]. (Cited on page 16.)

[223] Moussa, K.A., Clément, G. and Leygnac, C., "Black holes of topologically massive gravity", Class. Quantum Grav., 20, L277-L283, (2003). [DOI], [arXiv:gr-qc/0303042 [gr-qc]]. (Cited on page 16.)

[224] Murata, K., "Conformal weights in the Kerr/CFT correspondence", J. High Energy Phys., 2011(05), 117, (2011). [DOI], [arXiv:1103.5635 [hep-th]]. (Cited on pages 43, 50, and 51.)

[225] Nakayama, Y., "Emerging AdS from Extremally Rotating NS5-branes", Phys. Lett. B, 673, 272-278, (2009). [DOI], [arXiv:0812.2234 [hep-th]]. (Cited on pages 7, 20, and 35.)

[226] Nutku, Y., "Exact solutions of topologically massive gravity with a cosmological constant", Class. Quantum Grav., 10, 2657-2661, (1993). [DOI]. (Cited on page 16.)

[227] Ooguri, H., Strominger, A. and Vafa, C., "Black hole attractors and the topological string", Phys. Rev. D, 70, 106007, (2004). [DOI], [arXiv:hep-th/0405146]. (Cited on pages 14 and 17.)

[228] Ottewill, A.C. and Winstanley, E., "Divergence of a quantum thermal state on Kerr space-time", Phys. Lett. A, 273, 149-152, (2000). [DOI], [arXiv:gr-qc/0005108 [gr-qc]]. (Cited on page 23.)

[229] Ottewill, A.C. and Winstanley, E., "The Renormalized stress tensor in Kerr space-time: general results", Phys. Rev. D, 62, 084018, (2000). [DOI], [arXiv:gr-qc/0004022 [gr-qc]]. (Cited on page 23.)

[230] Pakman, A., Rastelli, L. and Razamat, S.S., "Diagrams for Symmetric Product Orbifolds", J. High Energy Phys., 2009(10), 034, (2009). [DOI], [arXiv:0905.3448 [hep-th]]. (Cited on page 30.)

[231] Penrose, R., "Asymptotic Properties of Fields and Space-Times", Phys. Rev. Lett., 10, 66-68, (1963). [DOI], [ADS]. (Cited on page 33.)

[232] Penrose, R., "Naked singularities", Ann. N.Y. Acad. Sci., 224, 125-134, (1973). [DOI]. (Cited on page 45.)

[233] Pioline, B. and Troost, J., "Schwinger pair production in $A d S_{2}$ ", J. High Energy Phys., 2005(03), 043, (2005). [DOI], [arXiv:hep-th/0501169 [hep-th]]. (Cited on page 48.)

[234] Polchinski, J., String Theory. Vol. 1: An Introduction to the Bosonic String, Cambridge Monographs on Mathematical Physics, (Cambridge University Press, Cambridge; New York, 1998). [Google Books]. (Cited on page 28.)

[235] Press, W.H. and Teukolsky, S.A., "Perturbations of a Rotating Black Hole. II. Dynamical Stability of the Kerr Metric", Astrophys. J., 185, 649-673, (1973). [DOI], [ADS]. (Cited on pages 44 and 49.)

[236] Rasmussen, J., "Isometry-preserving boundary conditions in the Kerr/CFT correspondence", Int. J. Mod. Phys. A, 25, 1597-1613, (2010). [DOI], [arXiv:0908.0184 [hep-th]]. (Cited on page 36.)

[237] Regge, T. and Teitelboim, C., "Role of surface integrals in the Hamiltonian formulation of general relativity", Ann. Phys. (N.Y.), 88, 286-318, (1974). [DOI]. (Cited on pages 32, 33, and 38.)

[238] Rooman, M. and Spindel, P., "Gödel metric as a squashed anti-de Sitter geometry", Class. Quantum Grav., 15, 3241-3249, (1998). [DOI], [gr-qc/9804027]. (Cited on page 16.)

[239] Seiberg, N., "Why is the matrix model correct?", Phys. Rev. Lett., 79, 3577-3580, (1997). [DOI], [arXiv:hep-th/9710009 [hep-th]]. (Cited on page 29.)

[240] Sen, A., "Black hole entropy function and the attractor mechanism in higher derivative gravity", J. High Energy Phys., 2005(09), 038, (2005). [DOI], [arXiv:hep-th/0506177 [hep-th]]. (Cited on pages 14 and 21.)

[241] Sen, A., "Logarithmic Corrections to Rotating Extremal Black Hole Entropy in Four and Five Dimensions", arXiv, e-print, (2011). [arXiv:1109.3706 [hep-th]]. (Cited on pages 21 and 63.) 
[242] Shao, K.-N. and Zhang, Z., "Hidden Conformal Symmetry of Rotating Black Hole with four Charges", Phys. Rev. D, 83, 106008, (2011). [DOI], [arXiv:1008.0585 [hep-th]]. (Cited on pages 43 and 52.)

[243] Sheikh-Jabbari, M.M. and Yavartanoo, H., "EVH Black Holes, AdS 3 Throats and EVH/CFT Proposal", J. High Energy Phys., 2011(10), 013, (2011). [DOI], [arXiv:1107.5705 [hep-th]]. (Cited on pages 7,16 , and 30.)

[244] Simón, J., "Extremal black holes, holography and coarse graining", Int. J. Mod. Phys. A, 26, 19031971, (2011). [DOI], [arXiv:1106.0116 [hep-th]]. (Cited on page 7.)

[245] Solodukhin, S.N., "Conformal description of horizon's states", Phys. Lett. B, 454, 213-222, (1999). [DOI], [arXiv:hep-th/9812056 [hep-th]]. (Cited on page 5.)

[246] Song, W. and Strominger, A., "D-brane Construction of the 5D NHEK Dual", arXiv, e-print, (2011). [arXiv:1105.0431 [hep-th]]. (Cited on pages 7 and 30.)

[247] Spradlin, M. and Strominger, A., "Vacuum states for $A d S_{2}$ black holes", J. High Energy Phys., 1999(11), 021, (1999). [DOI], [arXiv:hep-th/9904143 [hep-th]]. (Cited on page 25.)

[248] Starobinskii, A.A., "Amplification of waves during reflection from a rotating 'black hole", J. Exp. Theor. Phys., 37, 48-57, (1973). (Cited on page 44.)

[249] Starobinskii, A.A. and Churilov, S.M., "Amplification of electromagnetic and gravitational waves scattered by a rotating 'black hole"', J. Exp. Theor. Phys., 38, 3-11, (1974). (Cited on page 44.)

[250] Strominger, A., "Macroscopic entropy of $N=2$ extremal black holes", Phys. Lett. B, 383, 39-43, (1996). [DOI], [arXiv:hep-th/9602111 [hep-th]]. (Cited on page 14.)

[251] Strominger, A., "Black hole entropy from near-horizon microstates", J. High Energy Phys., 1998(02), 009, (1998). [DOI], [hep-th/9712251]. (Cited on pages 5 and 40.)

[252] Strominger, A., "AdS $S_{2}$ quantum gravity and string theory", J. High Energy Phys., 1999(01), 007, (1999). [DOI], [arXiv:hep-th/9809027 [hep-th]]. (Cited on page 29.)

[253] Strominger, A. and Vafa, C., "Microscopic Origin of the Bekenstein-Hawking Entropy", Phys. Lett. $B, 379,99-104$, (1996). [DOI], [arXiv:hep-th/9601029]. (Cited on page 5.)

[254] Sudarsky, D. and Wald, R.M., "Extrema of mass, stationarity, and staticity, and solutions to the Einstein-Yang-Mills equations", Phys. Rev., 46, 1453-1474, (1992). [DOI]. (Cited on page 10.)

[255] Teukolsky, S.A., "Rotating black holes: Separable wave equations for gravitational and electromagnetic perturbations", Phys. Rev. Lett., 29, 1114-1118, (1972). [DOI]. (Cited on page 44.)

[256] Teukolsky, S.A., "Perturbations of a rotating black hole. I. Fundamental equations for gravitational, electromagnetic, and neutrino-field perturbations", Astrophys. J., 185, 635-647, (1973). [DOI], [ADS]. (Cited on page 44.)

[257] Teukolsky, S.A. and Press, W.H., "Perturbations of a rotating black hole. III. Interaction of the hole with gravitational and electromagnetic radiation", Astrophys. J., 193, 443-461, (1974). [DOI]. (Cited on pages 44, 45, and 49.)

[258] Thorne, K.S., "Disk-accretion onto a black hole. II. Evolution of the hole", Astrophys. J., 191, 507-520, (1974). [DOI]. (Cited on page 5.)

[259] Townsend, P.K., "Black holes", arXiv, e-print, (1997). [arXiv:gr-qc/9707012]. (Cited on page 11.)

[260] Tripathy, P.K. and Trivedi, S.P., "Non-supersymmetric attractors in string theory", J. High Energy Phys., 2006(03), 022, (2006). [DOI], [arXiv:hep-th/0511117 [hep-th]]. (Cited on page 14.)

[261] Virmani, A., "Supertranslations and Holographic Stress Tensor", J. High Energy Phys., 2012(02), 024, (2012). [DOI], [arXiv:1112.2146 [hep-th]]. (Cited on page 33.) 
[262] Wald, R.M., "Black hole entropy is Noether charge", Phys. Rev., 48, R3427-R3431, (1993). [DOI], [arXiv:gr-qc/9307038]. (Cited on page 20.)

[263] Wang, Y.-Q. and Liu, Y.-X., "Hidden Conformal Symmetry of the Kerr-Newman Black Hole", J. High Energy Phys., 2010(08), 087, (2010). [DOI], [arXiv:1004.4661 [hep-th]]. (Cited on page 54.)

[264] Winstanley, E., "On classical super-radiance in Kerr-Newman-anti-de Sitter black holes", Phys. Rev. $D, \mathbf{6 4}, 104010,(2001)$. [DOI], [arXiv:gr-qc/0106032 [gr-qc]]. (Cited on page 11.)

[265] Witten, E., "Anti-de Sitter space and holography", Adv. Theor. Math. Phys., 2, 253-291, (1998). [arXiv:hep-th/9802150 [hep-th]]. (Cited on pages 5, 7, and 50.)

[266] Witten, E., "Multi-trace operators, boundary conditions, and AdS/CFT correspondence", arXiv, e-print, (2001). [arXiv:hep-th/0112258]. (Cited on page 38.)

[267] Witten, E., "SL $(2, Z)$ action on three-dimensional conformal field theories with Abelian symmetry", arXiv, e-print, (2003). [arXiv:hep-th/0307041]. (Cited on page 63.)

[268] Wu, X.-N. and Tian, Y., "Extremal Isolated Horizon/CFT Correspondence", Phys. Rev. D, 80, 024014, (2009). [DOI], [arXiv:0904.1554 [hep-th]]. (Cited on page 10.)

[269] Yano, K., "Some remarks on tensor fields and curvature", Ann. Math., 55, 328-347, (1952). [DOI]. Online version (accessed 1 May 2012):

http://www.jstor.org/stable/1969782. (Cited on page 45.)

[270] Youm, D., "Entropy of nonextreme rotating black holes in string theories", Nucl. Phys. B, 509, 431-460, (1998). [DOI], [arXiv:hep-th/9706046 [hep-th]]. (Cited on page 7.) 